\title{
Effectiveness of Antimicrobial Photodynamic Therapy in the Treatment of Periodontitis: A Systematic Review and Meta-Analysis of In Vivo Human Randomized Controlled Clinical Trials
}

\author{
Snehal Dalvi ${ }^{1,2, *(\mathbb{D})}$, Stefano Benedicenti ${ }^{1}$, Tudor Sălăgean ${ }^{3, *}$, Ioana Roxana Bordea ${ }^{4,+}$ (D) and Reem Hanna ${ }^{1,5,+}(\mathbb{D}$ \\ check for \\ updates \\ Citation: Dalvi, S.; Benedicenti, S.; \\ Sălăgean, T.; Bordea, I.R.; Hanna, R. \\ 1 Department of Surgical Sciences and Integrated Diagnostics, Laser Therapy Centre, University of Genoa, \\ Viale Benedetto XV, 6, 16132 Genoa, Italy; stefano.benedicenti@unige.it (S.B.); reemhanna@hotmail.com (R.H.) \\ 2 Department of Periodontology, Swargiya Dadasaheb Kalmegh Smruti Dental College and Hospital, \\ Nagpur 441110, India \\ 3 Department of Land Measurements and Exact Sciences, University of Agricultural Sciences and Veterinary \\ Medicine Cluj-Napoca, 400372 Cluj-Napoca, Romania \\ 4 Department of Oral Rehabilitation, "Iuliu Hațieganu” University of Medicine and Pharmacy Cluj-Napoca, \\ 400012 Cluj-Napoca, Romania; roxana.bordea@ymail.com \\ 5 Department of Oral Surgery, Dental Institute, King's College Hospital NHS Foundation Trust, \\ London SE5 9RS, UK \\ * Correspondence: drsnehaldeotale@gmail.com (S.D.); tudor.salagean@usamvcluj.ro (T.S.); \\ Tel.: +39-0-103-537-446 (S.D.); +40-744-707-371 (T.S.) \\ + These authors contributed equally to the last authorship.
} Effectiveness of Antimicrobial Photodynamic Therapy in the Treatment of Periodontitis: A Systematic Review and MetaAnalysis of In Vivo Human Randomized Controlled Clinical Trials. Pharmaceutics 2021, 13, 836. https://doi.org/10.3390/ pharmaceutics 13060836

Academic Editors:

Maria Nowakowska and Nejat Düzgünes

Received: 17 April 2021

Accepted: 2 June 2021

Published: 4 June 2021

Publisher's Note: MDPI stays neutral with regard to jurisdictional claims in published maps and institutional affiliations.

Copyright: (c) 2021 by the authors. Licensee MDPI, Basel, Switzerland. This article is an open access article distributed under the terms and conditions of the Creative Commons Attribution (CC BY) license (https:/ / creativecommons.org/licenses/by/ $4.0 /)$.

\begin{abstract}
This systematic review and meta-analysis evaluated antimicrobial photodynamic therapy (aPDT) efficacy in periodontitis. The review protocol was conducted in accordance with PRISMA statements, Cochrane Collaboration recommendations and is registered in PROSPERO (CRD 42020161516). Electronic and hand search strategies were undertaken to gather data on in vivo human RCTs followed by qualitative analysis. Differences in probing pocket depth (PPD) and clinical attachment level (CAL) were calculated with $95 \%$ confidence intervals and pooled in random effects model at three and six months. Heterogeneity was analyzed, using $Q$ and $\mathrm{I}^{2}$ tests. Publication bias was assessed by visual examination of the funnel plot symmetry. Sixty percent of 31 eligible studies showed a high risk of bias. Meta-analysis on 18 studies showed no additional benefit in split mouth studies in terms of PPD reduction (SMD $0.166 ; 95 \% \mathrm{CI}-0.278$ to $0.611 ; P=0.463$ ) and CAL gain (SMD 0.092; $95 \% \mathrm{CI}-0.013$ to 0.198; $P=0.088$ ). Similar findings noted for parallel group studies; PPD reduction (SMD 0.076; 95\% CI -0.420 to $0.573 ; P=0.763$ ) and CAL gain (SMD 0.056; $95 \%$ CI -0.408 to $0.552 ; P=0.745)$. Sensitivity analysis minimized heterogeneity for both outcome variables; however, intergroup differences were not statistically significant. Future research should aim for well-designed RCTs in order to determine the effectiveness of aPDT.
\end{abstract}

Keywords: antimicrobial photodynamic therapy; periodontitis; scaling and root planing; systematic review; meta-analysis

\section{Highlights}

1. Limitations of scaling and root planing (SRP) have directed the research to assess alternative comprehensive treatment strategies.

2. Antimicrobial Photodynamic therapy (aPDT) involves photo-excitation of photosensitizer dye upon illumination by a light of a matched wavelength.

3. This systematic review and meta-analysis evaluated the effectiveness of aPDT in the treatment of periodontitis.

4. In spite of the inconsistencies in their findings and methodological bias, the majority of the studies have demonstrated aPDT effectiveness. 
5. The efficacy of aPDT in improving treatment outcomes when it is utilized in the non-surgical management of periodontitis remains debatable.

\section{Introduction}

Antimicrobial Photodynamic therapy (aPDT) involves photo-excitation, which occurs when a photosensitizer (PS) dye is illuminated by a light of a matched wavelength, resulting in its activation and stimulation of a phototoxic response in the presence of ambient oxygen [1]. It has been persistently observed that bacterial recolonizations of Aggregatibacter actinomycetemcomitans (A.a) occur in periodontal pockets even after scaling and root planing (SRP) [2]. Aggressive periodontitis ( $\mathrm{AgP})$ is frequently associated with fewer local etiologic factors; therefore, it is believed that the affected patients are more likely to benefit from the antimicrobial effect of aPDT [3]. In contrast, chronic periodontitis (CP) patients usually have complex and thick deposits of polymicrobial communities on the affected root surfaces [4]. This may hamper penetration of PS, thereby reducing its effect and leading to an increase in the 'red complex' bacterial counts within a short period of time, resulting in a disease relapse [5]. Hence, the concept of replacing conventional SRP with aPDT is a controversial one with several imperative demerits, as enlisted above.

Utilization of adjunctive aPDT and its comparison with the gold standard SRP is a concept that has been studied extensively in both CP and AgP patients [6-16]. While SRP can quantitively lower the biomass of bacteria, aPDT has a more qualitative approach of a non-invasive nature, by creating alterations in cell membranes or Deoxyribonucleic Acid (DNA) damage [5]. Hence, a combination of these two therapies can be vouched for, since their mechanisms of action on microbiota and role in the periodontal repair process is distinct from the other and thus might have synergistic effects [17].

Distant sites of infection such as tonsils or base of tongue, which are affected due to the spread of tissue penetrating periopathogens, can be successfully reduced with local or systemic antibiotics (AB) [18]. Nonetheless, many clinicians often conduct NSPT without adjunctive $\mathrm{AB}$, which is only used when initial treatment has failed [19]. In AgP, evidence suggests that $\mathrm{SRP}+\mathrm{AB}$ therapy does not show satisfactory long-term results, unless reinstrumentation of affected sites is performed, as an additional step in the maintenance phase [20]. Furthermore, owing to the development of antibiotic resistant strains, it has been suggested that $A B$ usage should be restricted to those with a highly active disease or a specific microbiological profile [21]. In order to maintain an adequate mean inhibitory concentration (MIC) of any antimicrobial drug, either a sustained-release carrier medium is required or, conversely, a prompt bactericidal approach is needed to overcome the problem of physical displacement from the sulcus [22]. The aPDT falls into the latter category, demonstrating a 4-6-fold logarithmic bacterial reduction within a time frame of $60 \mathrm{~s}$ along with repeated applications [23]. A comprehensive assessment to evaluate the impact of these new trials on the role of aPDT in the treatment of periodontitis is unresolved, owing to the diversity in the methodology and results of existing scientific evidence [6-16].

In lieu of the prevailing pertinent literature, the present systematic review and metaanalysis aimed to provide a systematic evaluation of available scientific evidence to determine the efficacy of aPDT in the treatment of periodontitis. The objectives of this critical review were to evaluate the outcomes of this treatment strategy through various PS-laser wavelength combinations, as well as the laser parameters, in order to deduce an ideal PS-laser wavelength combination and treatment protocol for future scientific research.

\section{Materials and Methods}

\subsection{Protocol and Registration}

The present systematic review was reported based on the guidelines of Preferred Reporting Items for Systematic Reviews and Meta-Analysis (PRISMA) Statement and Cochrane Collaboration recommendations (Supplementary file 1) [24,25]. The review protocol is published in the Prospective Register of Systematic Reviews (PROSPERO); ref CRD 42020161516. 


\subsection{Population (P), Intervention (I), Comparison (C) and Outcomes (O)—PICO}

- Population: Patients diagnosed with Periodontitis (CP or $\mathrm{AgP})$ [26]

- Intervention: Utilisation of aPDT as a monotherapy or as an adjunct to SRP

- Comparison: Utilisation of SRP alone or SRP with adjunctive AB therapy

- Outcome: Evaluation of clinical and/or microbiological and/or immunological profiles

\subsection{Focused Research Question}

Is aPDT effective as a primary mode of treatment or as an adjunct to SRP compared to SRP alone or in combination with local or systemic antibiotics (AB), in terms of clinical or microbiological or immunological profiles, in patients with Periodontitis?

\subsection{Search Strategy}

The search strategy only included terms relating to or describing the study's domain and intervention. The use of relevant free text keywords and medical subject heading (Mesh) terms, which were logically connected with the help of Cochrane MEDLINE filters for controlled trials of interventions, was implemented. Individual search algorithms were developed for the following databases: MEDLINE (NCBI PubMed and PMC), Cochrane Central Register of Controlled Trials (CCRCT), Scopus, ScienceDirect, Google Scholar, EMBASE and EBSCO. Electronic search databases were searched thoroughly from their earliest records until 31 December 2019. The following journals were manually searched: Journal of Periodontology, Photomedicine and Laser Surgery, Clinical Oral Investigation, Journal of Clinical Periodontology, Journal of Dental Research, Lasers in Medical Science, Journal of Photochemistry and Photobiology and Photodiagnosis and Photodynamic Therapy. Related review articles and reference lists of all identified articles were searched through for further studies. Abstracts of the American Academy of Periodontology (AAP) and the European Federation of Periodontology (EFP) as well as sources for grey literature were screened to detect unpublished studies. In some instances, an attempt was made to establish a communication with the corresponding author in an attempt to obtain additional information related to the study; however, the attempts were unsuccessful. Search strategy was performed by two blinded, independent reviewers (S.D. and R.H.). In order to assess inter-reviewer reliability analysis, Kappa $(\mathrm{K})$ statistics were performed and a minimum value of 0.8 was considered acceptable [27]. In case of any disagreements, reviewers would discuss the discrepancies with a third author (S.B.), if necessary.

\subsection{Search Algorithms}

"Photodynamic therapy" OR "photochemotherapy"

AND

"Scaling" OR "Root planing" OR "non-surgical periodontal therapy"

AND

"Periodontitis" OR "Chronic Periodontitis" OR "Aggressive Periodontitis" OR “Early Onset Periodontitis"

\subsection{Eligibility Criteria}

\subsubsection{Inclusion Criteria}

1. Subjects diagnosed with CP or AgP according to 1999 AAP Classification of Periodontal diseases and conditions [26].

2. Studies included: In vivo human RCT's comparing the efficacy of aPDT in CP or AgP as monotherapy or adjunctive to SRP compared to SRP alone or in combination with AB.

3. Parallel group (PG) and split-mouth (SM) studies.

4. Age group $>18$ years, fit and healthy subjects.

5. No language restrictions for search strategy.

6. Studies that have utilized any PS dye (regardless dose and incubation period) and laser wavelength combination. 
7. Studies reporting at least one of the following parameters as an outcome variable: probing pocket depth (PPD), loss of clinical attachment level (CAL), bleeding on probing (BOP), plaque index (PI), gingival index (GI), microbiological profile, or immunological profile.

8. Studies with a minimum follow-up period of at least one month after treatment.

\subsubsection{Exclusion Criteria}

1. Subjects with systemic diseases or on medications that can influence the outcome variables.

2. Subjects who have undergone any periodontal therapy and/or antibiotic therapy in the last six months prior to RCT enrolment.

3. Studies utilizing low level laser therapy or laser therapy alone, as one of the intervention groups as compared to aPDT.

4. Studies involving utilization of aPDT for residual pockets or in supportive periodontal therapy (SPT).

5. Studies that have utilized light emitting diodes (LEDs) as a light source.

6. No outcome variable of interest.

7. Pregnancy.

8. Smoking.

9. Narrative and systematic reviews, in vitro studies, in vivo animal studies, commentaries, interviews, updates, case series and case reports.

\subsection{Systematic Review Outcomes}

2.7.1. Primary Outcome Measures

Changes in PPD and CAL from baseline up to the end of follow-up.

\subsubsection{Secondary Outcome Measures}

Changes in GR, BOP, PI, GI, microbiological and immunological profile from baseline up to the end of follow-up.

\subsection{Data Extraction}

Two reviewers independently (S.D. and R.H.) selected eligible studies from the search. They performed the review, assessment and data extraction for each eligible study. Each study received an identification with the name of the first author, year of publication and origin. A tabular representation of additional relevant information such as impact factor of journal, study design, sample size, demographics of the participants, baseline characteristics, intervention and comparator groups, type of photosensitizer used and dosage, laser parameters utilized, number of aPDT sessions performed, follow-up duration, statistical tests performed and results and conclusions, were gathered from each eligible study.

\subsection{Qualitative Analysis}

A qualitative assessment for each study was performed using the Revised Cochrane Risk-of-Bias (RoB) tool for Randomized trials, Version 2.0 (RoB 2) by two independent reviewers (S.D. and R.H.) [28-30]. Detailed assessment under the following headings was performed: 1. Bias arising from the randomization process; 2 . Bias due to deviations from intended interventions; 3 . Bias due to missing outcome data; 4 . Bias in measurement of the outcome; 5 . Bias in selection of the reported result. Depending upon fulfilment of above-mentioned criteria, the studies were determined as low, moderate or high RoB. Disagreements between the reviewers were resolved by discussion with a third author (S.B.) as well as use of 'discrepancy check' feature in RoB 2, in order to obtain consensus.

\subsection{Statistical Analysis of Data}

When appropriate and quantifiable data of interest were extracted from the eligible studies and combined for meta-analyses, using Stata version 15.1 software (StataCorp, Pyrmont, Australia), random effects meta-analyses were conducted to reflect the expected 
heterogeneity. As continuous outcomes were expected, overall treatment effects were calculated through pooled standardized mean differences (SMDs) with associated $95 \%$ confidence intervals (95\% CIs) for PPD and CAL. When information was presented in median and inter-quartile ranges, means and SDs were estimated [31]. Results from SM and PG studies were pooled separately at 3 and 6 months, respectively. A pooled overall effect was considered statistically significant when $p<0.05$. Consequently, statistical heterogeneity to identify outlier studies was performed by visual inspection of forest plots. Additionally, the Cochran $Q$ test was conducted to assess statistical heterogeneity $(p<0.10)$ [32]. $I^{2}$ statistics for homogeneity was expressed in a range of $0-100 \%$, with the following interpretation; $0 \%=$ no evidence of heterogeneity; $30-60 \%=$ moderate heterogeneity; $75-100 \%=$ high heterogeneity [33]. Sensitivity analysis was conducted to negate the effect of heterogeneity in between included studies by identifying the outlier studies by visual inspection of forest plots [34]. Publication bias was evaluated by visual assessment of funnel plot symmetry.

\section{Results}

\subsection{Study Selection}

Four hundred and sixty-two study titles were obtained from a combined electronic and manual search. Four study titles were obtained from cross-references. Therefore, a total of 466 study titles were included from all databases in the preliminary screening (inter-reviewer agreement, $\mathrm{k}=0.9$ ). Three hundred and eighty-seven articles were excluded, due to duplication and the remaining 79 records were further evaluated (inter-reviewer agreement, $\mathrm{k}=0.94)$. Twelve articles were excluded based on their titles and abstracts, mainly due to an inappropriate study design (inter-reviewer agreement, $k=0.92$ ). Thus, 67 articles were assessed for their eligibility. These articles were evaluated based on eligibility criteria. Additionally, 36 studies were excluded due to following reasons: Smokers were included or smoking details were not provided in 12 studies [23,35-45]; Laser or LLLT was utilized, as an adjunct to SRP in eight studies [46-53]; LED-aPDT was performed in seven studies [54-60]; aPDT was used in management of residual pockets in four studies [61-64] and as an adjunct to supportive periodontal therapy in two studies [65,66]; patients with systemic diseases were included in two studies $[67,68]$, whereas one study did not perform a follow-up assessment [69] (inter-reviewer agreement, $k=1$ ). Hence, out of 67 full text articles, 31 articles were included and analyzed in the present systematic review [2,3,5,17,70-96]. All included articles were in vivo human studies. A meta-analysis on 18 out of 31 studies which assessed efficacy of SRP+aPDT was conducted $[17,71,73-76,80,81,84-86,88-93,96]$ (inter-reviewer agreement, $k=1$ ). Figure 1 depicts the PRISMA flow diagram for search strategy utilized in the present systematic review and meta-analysis.

\subsection{Study Characteristics}

\subsubsection{Country of Origin}

A substantial diversity in the country of origin was noted amongst included papers (Table 1). Distribution of studies was as follows: 11 in Brazil [2,3,5,17,71,82,83,87,92,95,96], 6 in India $[78,81,88,89,91,93], 4$ in Germany $[76,77,80,94], 4$ in Iran $[70,85,86,90], 3$ in Poland [72-74], whereas there is 1 study each, in the following countries; Spain [75], Japan [79], Thailand [84]. 


\section{PRISMA FLOW DIAGRAM FOR SELECTION OF STUDIES}
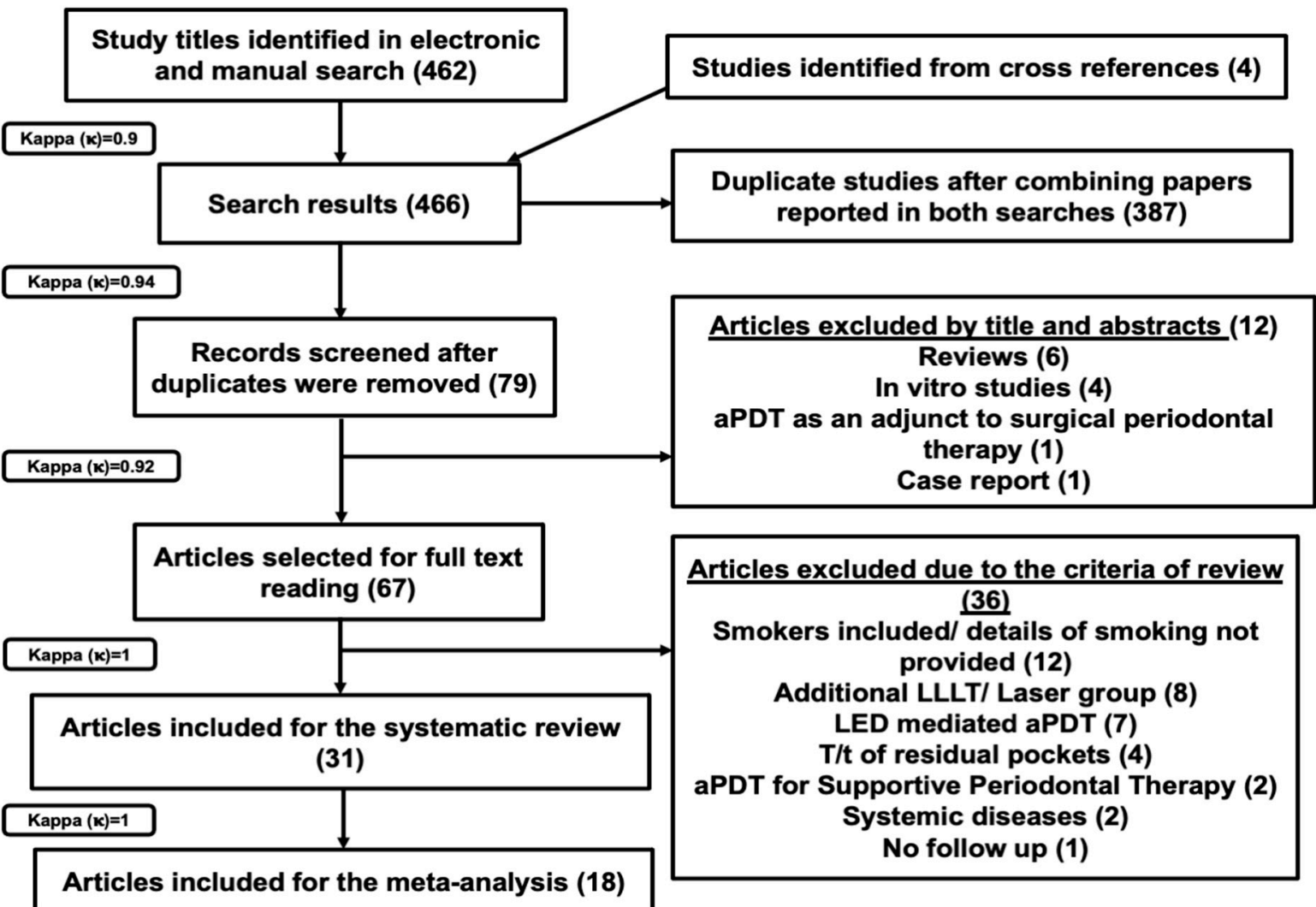

Articles excluded due to the criteria of review (36)

Smokers included/ details of smoking not provided (12)

Additional LLLT/ Laser group (8)

LED mediated aPDT (7)

T/t of residual pockets (4)

aPDT for Supportive Periodontal Therapy (2)

Systemic diseases (2)

No follow up (1)

Figure 1. PRISMA flow diagram of the study selection criteria.

\subsubsection{Study Design}

Twenty studies were conducted using a SM study design $[2,3,5,17,70,71,77,80-82$, 84-87,89,90,92-95], whereas a PG study design was utilized in the remaining 11 studies $[72-76,78,79,83,88,91,96]$ (Table 1).

\subsubsection{Selection Criteria}

Several inconsistencies were observed amongst the included studies [2,3,5,17,70-96], which have been outlined in Table 1, in which 21 out of 31 studies included patients with CP $[17,70,75-82,84,86,88-96]$, whereas the remaining 10 studies included patients with $\operatorname{AgP}[2,3,5,71-74,83,85,87]$. 


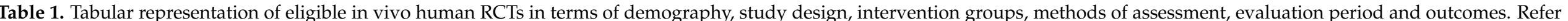
to Supplementary file 2 for list of abbreviations.

\begin{tabular}{|c|c|c|c|c|c|c|c|c|c|c|c|}
\hline $\begin{array}{l}\text { Study, Year, } \\
\text { Origin and } \\
\text { Citation }\end{array}$ & $\begin{array}{l}\text { Journal Name/ } \\
\text { Impact Factor } \\
\text { (IF) }\end{array}$ & $\begin{array}{c}\text { Study } \\
\text { Design }\end{array}$ & Type of Periodontitis & $\begin{array}{l}\text { Sample } \\
\text { Size }(n)\end{array}$ & $\begin{array}{l}\text { Gender } \\
\mathbf{M} / \mathbf{F}\end{array}$ & $\begin{array}{c}\text { Age (Years) } \\
\text { (Mean } \pm \text { SD) }\end{array}$ & \multicolumn{2}{|c|}{ Intervention Groups } & $\begin{array}{l}\text { Evaluation } \\
\text { Period }\end{array}$ & $\begin{array}{l}\text { Parameters } \\
\text { Assessed }\end{array}$ & Conclusions \\
\hline $\begin{array}{l}\text { De Oliveira } \\
\text { et al., 2009 } \\
\text { (Brazil) [2] }\end{array}$ & $\begin{array}{l}\text { Journal of Peri- } \\
\text { odontology } \\
\text { IF 2020: } 3.742 \\
\text { IF 2009: } 2.580\end{array}$ & SM-RCT & $\begin{array}{l}\mathrm{AgP} \\
\text { (A minimum of } 20 \text { teeth (mean, } \\
26 \text { teeth) with at least one tooth } \\
\text { in each posterior sextant and at } \\
\text { least one posterior sextant with } \\
\text { a minimum of three natural } \\
\text { teeth; } \geq 5 \mathrm{~mm} \text { of attachment loss } \\
\text { around at least seven teeth } \\
\text { involved, excluding first molars } \\
\text { and central incisors) }\end{array}$ & 10 & $2 / 8$ & $\begin{array}{c}\text { 18-35 } \\
\text { Mean: } \\
31.01 \pm 4.43\end{array}$ & $\begin{array}{c}\text { SRP } \\
\text { (Hand instruments) } \\
\text { (10 teeth) }\end{array}$ & $\begin{array}{c}\text { aPDT } \\
\text { (10 teeth) }\end{array}$ & $\begin{array}{l}-7 \text { (baseline), } \\
0 \\
\text { (immediately } \\
\text { after interven- } \\
\text { tions) },+1 \\
+7,+30 \text { and } \\
+90 \text { days. }\end{array}$ & $\begin{array}{l}\text { TNF- } \alpha \text { and } \\
\text { RANKL } \\
\text { assessment }\end{array}$ & $\begin{array}{c}\text { NSPT with PDT or SRP led to } \\
\text { statistically significant reductions } \\
\text { in TNF-a level } 30 \text { days following } \\
\text { treatment }(p<0.05) \text { with no } \\
\text { statistically significant intergroup } \\
\text { differences }(p>0.5) \text {. }\end{array}$ \\
\hline $\begin{array}{l}\text { De Oliveira } \\
\text { et al., 2007 } \\
\text { (Brazil) [3] }\end{array}$ & $\begin{array}{l}\text { Journal of Peri- } \\
\text { odontology } \\
\text { IF 2020: } 3.742 \\
\text { IF 2007: } 2.426\end{array}$ & SM-RCT & $\begin{array}{c}\mathrm{AgP} \\
\text { (A minimum of } 20 \text { teeth (mean, } \\
26 \text { teeth) with at least one tooth } \\
\text { in each posterior sextant and at } \\
\text { least one posterior sextant with } \\
\text { a minimum of three natural } \\
\text { teeth; } \geq 5 \mathrm{~mm} \text { of attachment loss } \\
\text { around at least seven teeth } \\
\text { involved, excluding first molars } \\
\text { and central incisors) }\end{array}$ & 10 & $2 / 8$ & $\begin{array}{c}\text { 18-35 } \\
\text { Mean: } \\
31.01 \pm 4.43\end{array}$ & $\begin{array}{c}\text { SRP } \\
\text { (Hand instruments) } \\
\text { (10 teeth) }\end{array}$ & $\begin{array}{c}\text { aPDT } \\
\text { (10 teeth) }\end{array}$ & $\begin{array}{l}\text { Baseline, } 3 \\
\text { months }\end{array}$ & $\begin{array}{l}\text { PD, RCAL, GR, } \\
\text { PI, GI, BOP }\end{array}$ & $\begin{array}{l}\text { PDT and SRP showed statistically } \\
\text { significant clinical results }(p<0.05) \\
\text { in the non-surgical treatment of } \\
\text { aggressive periodontitis with no } \\
\text { statistically significant differences } \\
(p>0.5) \text { in intergroup comparison. }\end{array}$ \\
\hline $\begin{array}{c}\text { Novaes et al., } \\
2012 \\
\text { (Brazil) [5] }\end{array}$ & $\begin{array}{l}\text { Lasers in } \\
\text { Medical } \\
\text { Science } \\
\text { IF 2019: } 2.574 \\
\text { IF 2012: } 2.645\end{array}$ & SM-RCT & $\begin{array}{l}\mathrm{AgP} \\
\text { (A minimum of } 20 \text { teeth (mean, } \\
26 \text { teeth) with at least one tooth } \\
\text { in each posterior sextant, and at } \\
\text { least one posterior sextant with } \\
\text { a minimum of three natural } \\
\text { teeth; } \geq 5 \mathrm{~mm} \text { of attachment loss } \\
\text { around at least seven teeth } \\
\text { involved, excluding first molars } \\
\text { and central incisors) }\end{array}$ & 10 & $2 / 8$ & $\begin{array}{c}18-35 \\
\text { Mean: } 31\end{array}$ & $\begin{array}{c}\text { SRP } \\
\text { (Hand instruments) }\end{array}$ & aPDT & $\begin{array}{c}-7,0 \\
\text { (Baseline), } \\
\text { and } 3 \text { months }\end{array}$ & $\begin{array}{l}\text { Plaque sample } \\
\text { analysis for } \\
\text { estimation of } \\
40 \text { subgingival } \\
\text { species using } \\
\text { DNA-DNA } \\
\text { hybridization. }\end{array}$ & $\begin{array}{l}\text { aPDT was more effective in } \\
\text { reducing the counts of } A \cdot a \\
(p=0.00) \text { whereas, SRP reduced } \\
\text { red complex bacteria. } \\
\text { Combination of both treatment } \\
\text { methods would be beneficial for } \\
\text { the non-surgical treatment of AgP }\end{array}$ \\
\hline $\begin{array}{c}\text { Franco et al., } \\
2014 \\
\text { (Brazil) [17] }\end{array}$ & $\begin{array}{c}\text { Photodiagnosis } \\
\text { and } \\
\text { Photodynamic } \\
\text { Therapy } \\
\text { IF 2020: } 2.894 \\
\text { IF 2014: } 2.359\end{array}$ & SM-RCT & $\begin{array}{l}\mathrm{CP} \\
\text { (At least } 20 \text { teeth with at least } \\
\text { one posterior tooth in each } \\
\text { quadrant, and periodontal } \\
\text { pockets } \geq 5 \mathrm{~mm} \text { on at least } \\
\text { seven teeth) }\end{array}$ & 15 & NI & 39.5 & $\begin{array}{c}\text { SRP } \\
\text { (Hand instruments) }\end{array}$ & $\mathrm{SRP}+\mathrm{aPDT}$ & $\begin{array}{l}\text { Baseline and } \\
90 \text { days }\end{array}$ & $\begin{array}{l}\text { BOP, PI, PD, } \\
\text { CAL, qPCR } \\
\text { gene expression } \\
\text { analysis. }\end{array}$ & $\begin{array}{l}\text { Significant improvement in BOP } \\
\text { was noted with aPDT group } \\
(p=0.03) \text {. PDT increased the } \\
\text { expression of RANK and OPG, } \\
\text { which could indicate a reduction } \\
\text { in osteoclastogenesis. } \\
\text { Furthermore, the use of PDT in } \\
\text { conjunction with conventional } \\
\text { treatment significantly increased } \\
\text { the expression of FGF2, which has } \\
\text { an important role in the } \\
\text { periodontal repair process. }\end{array}$ \\
\hline
\end{tabular}


Table 1. Cont.

\begin{tabular}{|c|c|c|c|c|c|c|c|c|c|c|c|}
\hline $\begin{array}{l}\text { Study, Year, } \\
\text { Origin and } \\
\text { Citation }\end{array}$ & $\begin{array}{l}\text { Journal Name/ } \\
\text { Impact Factor } \\
\text { (IF) }\end{array}$ & $\begin{array}{c}\text { Study } \\
\text { Design }\end{array}$ & Type of Periodontitis & $\begin{array}{l}\text { Sample } \\
\text { Size }(n)\end{array}$ & $\begin{array}{l}\text { Gender } \\
M / F\end{array}$ & $\begin{array}{c}\text { Age (Years) } \\
(\text { Mean } \pm \text { SD) }\end{array}$ & \multicolumn{2}{|c|}{ Intervention Groups } & $\begin{array}{l}\text { Evaluation } \\
\text { Period }\end{array}$ & $\begin{array}{l}\text { Parameters } \\
\text { Assessed }\end{array}$ & Conclusions \\
\hline $\begin{array}{l}\text { Pourabbas } \\
\text { et al., 2014 } \\
\text { (Iran) [70] }\end{array}$ & $\begin{array}{l}\text { Journal of Peri- } \\
\text { odontology } \\
\text { IF 2020: } 3.742 \\
\text { IF 2014: } 2.900\end{array}$ & SM-RCT & $\begin{array}{c}\text { CP } \\
\text { ( } \geq 12 \text { natural teeth with a } \\
\text { minimum of three in each } \\
\text { quadrant; } \geq 3 \mathrm{~mm} \text { attachment } \\
\text { loss in about a minimum of } \\
30 \% \text { of the existing teeth; } \\
\geq 1 \text { site per quadrant with } \\
\text { PPD of } \geq 4 \text { mm and BOP) }\end{array}$ & 24 & $10 / 14$ & $46 \pm 8$ & $\begin{array}{c}\text { SRP } \\
\text { (Sonic and hand } \\
\text { instruments) }\end{array}$ & $\mathrm{SRP}+\mathrm{aPDT}$ & $\begin{array}{l}\text { Baseline and } \\
3 \text { months }\end{array}$ & $\begin{array}{l}\text { PD, BOP, CAL, } \\
\text { GR, IL-1 } \beta \\
\text { TNF- } \alpha, \text { MMP- } 8 \\
\text { and MMP-9 } \\
\text { analysis }\end{array}$ & $\begin{array}{l}\text { Intragroup comparison showed } \\
\text { significant improvements } \\
(p<0.001) \text { for all variables in } \\
\text { 3-month follow-up compared with } \\
\text { baseline. TNF- } \alpha \text { was significantly } \\
\text { improved in the SRP+aPDT versus } \\
\text { SRP group }(p<0.001) \text {. Total levels } \\
\text { of PMNs were reduced for all } \\
\text { patients compared with baseline } \\
\text { levels }(p<0.001) \text {. }\end{array}$ \\
\hline $\begin{array}{l}\text { Moreira et al., } \\
2015 \\
\text { (Brazil) [71] }\end{array}$ & $\begin{array}{l}\text { Journal of Peri- } \\
\text { odontology } \\
\text { IF 2020: } 3.742 \\
\text { IF 2015: } 3.159\end{array}$ & SM-RCT & $\begin{array}{l}\mathrm{AgP} \\
\text { (A minimum of } 20 \text { teeth and } \\
\text { two pairs of single rooted } \\
\text { contralateral teeth with } \\
\text { proximal sites presenting PD } \\
\text { and CAL } \geq 5 \mathrm{~mm} \text { ) }\end{array}$ & 20 & $2 / 18$ & $\begin{array}{c}18-35 \\
30.6 \pm 4.25\end{array}$ & $\begin{array}{c}\text { SRP }+ \text { sham } \\
\text { procedure } \\
\text { (Hand and ultrasonic } \\
\text { instruments) } \\
40 \text { teeth } / 128 \text { sites }\end{array}$ & $\begin{array}{l}\text { SRP+aPDT } \\
40 \text { teeth/ } \\
135 \text { sites }\end{array}$ & $\begin{array}{l}\text { Baseline, } \\
3 \text { months }\end{array}$ & $\begin{array}{c}\text { PD, CAL, GR, } \\
\text { PI, BOP } \\
\text { Microbiological } \\
\text { analysis for } \\
\text { counts of } \\
40 \text { bacterial } \\
\text { species using } \\
\text { DNA- DNA } \\
\text { Hybridization } \\
\text { Immunological } \\
\text { evaluation for } \\
\text { GCF levels of } \\
\text { IL-1 } \beta, \text { IL- } 10 \\
\text { and TNF- } \alpha \text {. }\end{array}$ & $\begin{array}{c}\text { In deep periodontal pockets } \\
\text { analysis (PD } \geq 7 \mathrm{~mm} \text { at baseline), } \\
\text { Test Group presented a decrease in } \\
\mathrm{PD} \text { and a clinical attachment gain } \\
\text { significantly higher than Control } \\
\text { Group at } 90 \text { days }(p<0.05) \text {. Test } \\
\text { Group also demonstrated } \\
\text { significantly less periodontal } \\
\text { pathogens of red and orange } \\
\text { complexes and a lower ratio } \\
\text { IL-1 } \beta / \mathrm{IL}-10 \text { than Control Group } \\
(p<0.05) \text {. Four adjunctive sessions } \\
\text { of aPDT after SRP have clinical, } \\
\text { microbiological and } \\
\text { immunological benefits over SRP } \\
\text { alone in management of AgP. }\end{array}$ \\
\hline $\begin{array}{l}\text { Skurska et al., } \\
2015 \\
\text { (Poland) [72] }\end{array}$ & $\begin{array}{l}\text { BMC Oral } \\
\text { Health } \\
\text { IF 2019: } 1.911 \\
\text { IF 2015: } 1.605\end{array}$ & PG-RCT & $\begin{array}{c}\mathrm{AgP} \\
\text { (At least } 3 \text { sites with } \\
\mathrm{PD} \geq 6 \mathrm{~mm} \text { ) }\end{array}$ & $\begin{array}{c}35 \\
\text { SRP+AB: } \\
17 \\
\text { SRP+aP } \\
\text { DT: } 18\end{array}$ & $\begin{array}{c}12 / 24 \\
\text { SRP+aPDT: } \\
7 / 10 \\
\text { SRP+AB: } \\
5 / 13\end{array}$ & $\begin{array}{c}23-55 \\
\text { SRP+aPDT: } \\
37.3 \pm 8.0 \\
\text { SRP+AB: } \\
34.7 \pm 9.0\end{array}$ & $\begin{array}{c}\text { SRP }+\mathrm{AB} \\
141 \text { sites } \\
\text { AB: } \\
375 \text { mg of amoxicillin } \\
+250 \mathrm{mg} \text { of } \\
\text { metronidazole TDS } \\
\text { for } 7 \text { days, starting on } \\
\text { the day of SRP } \\
\text { (Hand and ultrasonic } \\
\text { instruments) }\end{array}$ & $\begin{array}{l}\text { SRP+aPDT } \\
137 \text { sites }\end{array}$ & $\begin{array}{l}\text { Baseline, } 3 \\
\text { and } 6 \text { months }\end{array}$ & $\begin{array}{l}\text { MMP-8 and } \\
\text { MMP-9 } \\
\text { assessment }\end{array}$ & $\begin{array}{l}\text { In the AB group, patients showed } \\
\text { a statistically significant }(p=0.01) \\
\text { decrease of MMP-8 GCF level at } \\
\text { both 3- and 6-months post } \\
\text { treatment. In the PDT group, the } \\
\text { change of MMP-8 GCF level was } \\
\text { not statistically significant. Both } \\
\text { groups showed at } 3 \text { and } 6 \text { months } \\
\text { a decrease in MMP-9 levels. } \\
\text { However, this change did not } \\
\text { reach statistical significance. } \\
\text { SRP+AB is more effective in } \\
\text { reducing GCF MMP-8 levels } \\
\text { compared to SRP+aPDT. }\end{array}$ \\
\hline
\end{tabular}


Table 1. Cont.

\begin{tabular}{|c|c|c|c|c|c|c|c|c|c|c|c|}
\hline $\begin{array}{l}\text { Study, Year, } \\
\text { Origin and } \\
\text { Citation }\end{array}$ & $\begin{array}{l}\text { Journal Name/ } \\
\text { Impact Factor } \\
\text { (IF) }\end{array}$ & $\begin{array}{l}\text { Study } \\
\text { Design }\end{array}$ & Type of Periodontitis & $\begin{array}{l}\text { Sample } \\
\text { Size }(n)\end{array}$ & $\begin{array}{l}\text { Gender } \\
\mathbf{M} / \mathbf{F}\end{array}$ & $\begin{array}{c}\text { Age (Years) } \\
\text { (Mean } \pm \text { SD) }\end{array}$ & \multicolumn{2}{|c|}{ Intervention Groups } & $\begin{array}{l}\text { Evaluation } \\
\text { Period }\end{array}$ & $\begin{array}{l}\text { Parameters } \\
\text { Assessed }\end{array}$ & Conclusions \\
\hline $\begin{array}{l}\text { Arweiler } \\
\text { et al., 2014 } \\
\text { (Poland) [73] }\end{array}$ & $\begin{array}{l}\text { Clinical Oral } \\
\text { Investigations } \\
\text { IF 2019: } 2.903 \\
\text { IF 2014: } 2.704\end{array}$ & PG-RCT & $\begin{array}{c}\text { AgP } \\
\text { (At least } 3 \text { sites with } \\
\mathrm{PD} \geq 6 \mathrm{~mm} \text { ) }\end{array}$ & $\begin{array}{c}35 \\
\text { SRP+aPDT: } \\
17 \\
\text { SRP+AB: } \\
18\end{array}$ & $\begin{array}{c}12 / 24 \\
\text { SRP+aPDT: } \\
7 / 10 \\
\text { SRP+AB: } \\
5 / 13\end{array}$ & $\begin{array}{l}23-55 \\
\text { SRP+aPDT: } \\
37.3 \pm 8.0 \\
\text { SRP+AB: } \\
34.7 \pm 9.0\end{array}$ & $\begin{array}{c}\text { SRP+AB } \\
141 \text { sites } \\
\text { AB: } \\
375 \mathrm{mg} \text { Amoxicillin }+ \\
250 \mathrm{mg} \text { Metronidazole } \\
\text { TDS for } 7 \text { days } \\
\text { (starting from day } \\
\text { of SRP) } \\
\text { (Hand and ultrasonic } \\
\text { instruments) }\end{array}$ & $\begin{array}{l}\text { SRP+aPDT } \\
137 \text { sites }\end{array}$ & $\begin{array}{l}\text { Baseline, } \\
6 \text { months }\end{array}$ & $\begin{array}{l}\text { PD, CAL, GR, } \\
\text { PI, BOP, FMPI, } \\
\text { FMBOP }\end{array}$ & $\begin{array}{c}\text { Intragroup comparison revealed } \\
\text { statistically significant PD } \\
\text { reduction from baseline }(p<0.001) \\
\text { SRP }+\mathrm{AB} \text { showed significant } \\
\text { differences in PD reduction and } \\
\text { lower number of deep } \\
\text { pockets } \geq 7 \mathrm{~mm}(p<0.001) \text { as } \\
\text { compared to SRP+aPDT }(p=0.03) \text {. }\end{array}$ \\
\hline $\begin{array}{l}\text { Arweiler } \\
\text { et al., 2013 } \\
\text { (Poland) [74] }\end{array}$ & $\begin{array}{l}\text { Schweiz } \\
\text { Monatsschr } \\
\text { Zahnmed } \\
\text { IF 2020: NA } \\
\text { IF 2013: NA }\end{array}$ & PG-RCT & $\begin{array}{c}\mathrm{AgP} \\
\text { (At least } 3 \text { sites with } \\
\mathrm{PD} \geq 6 \mathrm{~mm} \text { ) }\end{array}$ & $\begin{array}{c}35 \\
\text { SRP+aPDT: } \\
17 \\
\text { SRP+AB: } \\
18\end{array}$ & $\begin{array}{c}12 / 24 \\
\text { SRP+aPDT: } \\
7 / 10 \\
\text { SRP+AB: } \\
5 / 13\end{array}$ & $\begin{array}{l}23-55 \\
\text { SRP+aPDT: } \\
37.3 \pm 8.0 \\
\text { SRP+AB: } \\
34.7 \pm 9.0\end{array}$ & $\begin{array}{c}\mathrm{SRP}+\mathrm{AB} \\
141 \text { sites } \\
\mathrm{AB}: \\
375 \mathrm{mg} \\
\text { Amoxicillin+250 mg } \\
\text { MTZ } \\
\text { TDS for } 7 \text { days } \\
\text { (starting from day of } \\
\text { SRP) } \\
\text { (Hand and ultrasonic } \\
\text { instruments) }\end{array}$ & $\begin{array}{l}\text { SRP+aPDT } \\
137 \text { sites }\end{array}$ & $\begin{array}{l}\text { Baseline, } \\
3 \text { months }\end{array}$ & $\begin{array}{l}\text { PD, CAL, GR, } \\
\text { PI, BOP, FMPI, } \\
\text { FMBOP }\end{array}$ & $\begin{array}{c}\mathrm{SRP}+\mathrm{AB} \text { showed significant } \\
\text { differences in PD reduction, } \mathrm{CAL} \\
\text { gain and lower number of deep } \\
\text { pockets } \geq 7 \mathrm{~mm} \text { as compared to } \\
\text { SRP+aPDT }(p<0.001) \text {. }\end{array}$ \\
\hline $\begin{array}{l}\text { Vidal et al., } \\
2017 \\
\text { (Spain) [75] }\end{array}$ & $\begin{array}{l}\text { Journal of } \\
\text { Clinical Peri- } \\
\text { odontology } \\
\text { IF 2020: } 5.241 \\
\text { IF 2017: } 4.165\end{array}$ & PG-RCT & $\begin{array}{c}\mathrm{CP} \\
\text { (Four or more periodontal } \\
\text { pockets with a PPD } \geq 5 \mathrm{~mm} \\
\text { and BOP) }\end{array}$ & 37 & $11 / 26$ & $55 \pm 2$ & $\begin{array}{c}\text { SRP } \\
\text { (Hand and ultrasonic } \\
\text { instruments) }\end{array}$ & $\mathrm{SRP}+\mathrm{aPDT}$ & $\begin{array}{l}\text { Baseline, } 5,13 \\
\text { and } 25 \text { weeks }\end{array}$ & $\begin{array}{c}\text { PI, PD, GR, } \\
\text { CAL, BOP, GCF } \\
\text { volume, } \\
\text { microbiological } \\
\text { and } \\
\text { biochemical } \\
\text { parameters }\end{array}$ & $\begin{array}{l}\text { RANKL and abundance of } A . a \text { was } \\
\text { significantly decreased in the } \\
\text { SRP+aPDT group compared with } \\
\text { the SRP group }(p<0.05) \text {. Except of } \\
\text { a reduction in } A . a, \text { SRP+ aPDT } \\
\text { resulted in no additional } \\
\text { improvement compared with } \\
\text { SRP alone. }\end{array}$ \\
\hline $\begin{array}{l}\text { Braun et al., } \\
2008 \text { (Ger- } \\
\text { many) [76] }\end{array}$ & $\begin{array}{l}\text { Journal of } \\
\text { Clinical Peri- } \\
\text { odontology } \\
\text { IF 2020: } 5.241 \\
\text { IF 2008: } 3.525\end{array}$ & SM-RCT & $\begin{array}{l}\mathrm{CP} \\
\text { (At least one premolar and } \\
\text { one molar in every quadrant } \\
\text { with a minimum of four } \\
\text { teeth each; at least one tooth } \\
\text { with an attachment loss of } \\
>3 \mathrm{~mm} \text { in every quadrant) }\end{array}$ & 20 & $9 / 11$ & $46.6 \pm 6.1$ & $\begin{array}{l}\text { SRP } \\
\text { (Hand and piezo- } \\
\text { electric ultrasonic } \\
\text { instruments) }\end{array}$ & $\mathrm{SRP}+\mathrm{aPDT}$ & $\begin{array}{l}\text { Baseline, } \\
1 \text { week, } \\
3 \text { months }\end{array}$ & $\begin{array}{l}\text { SFFR, BOP, RAL } \\
\text { PD, GR }\end{array}$ & $\begin{array}{l}\text { Values for RAL, PD, SFFR and } \\
\text { BOP decreased significantly } \\
3 \text { months after treatment in the } \\
\text { control group with a higher impact } \\
\text { on the sites treated with adjunctive } \\
\text { aPDT ( } p<0.05) \text {. GR increased } \\
3 \text { months after treatment with and } \\
\text { without adjunctive aPDT, with no } \\
\text { difference between the groups } \\
(p>0.05) \text {. In patients with CP, } \\
\text { clinical outcomes can be improved } \\
\text { by adjunctive aPDT. }\end{array}$ \\
\hline
\end{tabular}


Table 1. Cont

\begin{tabular}{|c|c|c|c|c|c|c|c|c|c|c|c|}
\hline $\begin{array}{l}\text { Study, Year, } \\
\text { Origin and } \\
\text { Citation }\end{array}$ & $\begin{array}{l}\text { Journal Name/ } \\
\text { Impact Factor } \\
\text { (IF) }\end{array}$ & $\begin{array}{l}\text { Study } \\
\text { Design }\end{array}$ & Type of Periodontitis & $\begin{array}{l}\text { Sample } \\
\text { Size }(n)\end{array}$ & $\begin{array}{l}\text { Gender } \\
\text { M/F }\end{array}$ & $\begin{array}{c}\text { Age (Years) } \\
\text { (Mean } \pm \text { SD) }\end{array}$ & Intervention Gr & & $\begin{array}{l}\text { Evaluation } \\
\text { Period }\end{array}$ & $\begin{array}{l}\text { Parameters } \\
\text { Assessed }\end{array}$ & Conclusions \\
\hline $\begin{array}{l}\text { Berakdar } \\
\text { et al., } 2012 \\
\quad(\text { Ger- } \\
\text { many) [77] }\end{array}$ & $\begin{array}{l}\text { Head and Face } \\
\text { Medicine } \\
\text { IF 2020: } 1.492 \\
\text { IF 2012: } 1.519\end{array}$ & SM-RCT & $\begin{array}{l}\mathrm{CP} \\
\text { (At least four teeth with a } \\
\text { PPD of } \geq 5 \mathrm{~mm} \text { ) }\end{array}$ & 22 & $12 / 10$ & $59.3 \pm 11.7$ & $\begin{array}{c}\text { SRP } \\
\text { (Hand instruments) }\end{array}$ & $\mathrm{SRP}+\mathrm{aPDT}$ & $\begin{array}{l}\text { Baseline, } 1,3 \\
\text { and } 6 \text { months }\end{array}$ & $\begin{array}{l}\text { BOP, PI, PD, } \\
\text { CAL }\end{array}$ & $\begin{array}{l}\text { At } 1,3 \text { and } 6 \text { months after both } \\
\text { types of treatment, an } \\
\text { improvement in BOP and CAL } \\
\text { was observed. The greater } \\
\text { reduction of the PD, achieved by a } \\
\text { combination of SRP /PDT, was } \\
\text { statistically significant after } \\
6 \text { months }(p=0.007) .\end{array}$ \\
\hline $\begin{array}{l}\text { Raut et al., } \\
2018 \\
\text { (India) [78] }\end{array}$ & $\begin{array}{c}\text { Journal of } \\
\text { Indian Society } \\
\text { of Periodontol- } \\
\text { ogy } \\
\text { IF 2020: } 0.460 \\
\text { IF 2018: } 0.44\end{array}$ & PG-RCT & $\begin{array}{c}\mathrm{CP} \\
(\mathrm{PPD}>5 \mathrm{~mm} \text { and } \mathrm{CAL}>4 \mathrm{~mm})\end{array}$ & 50 & $\begin{array}{l}\text { SRP group: } \\
\text { 12/13 } \\
\text { SRP+aPDT } \\
\text { group: } \\
16 / 9\end{array}$ & $\begin{array}{l}\text { SRP group: } \\
46.90 \pm 4.32 \\
\text { SRP+aPDT } \\
\text { group: } \\
51 \pm 2.83\end{array}$ & $\begin{array}{l}\text { SRP+ sham procedure } \\
\text { (Hand and ultrasonic } \\
\text { instruments) }\end{array}$ & $\mathrm{SRP}+\mathrm{aPDT}$ & $\begin{array}{l}\text { Baseline and } \\
6 \text { months }\end{array}$ & $\begin{array}{l}\text { PI, BOP, CAL, } \\
\text { PD, } \\
\text { microbiological } \\
\text { analysis }\end{array}$ & $\begin{array}{l}\text { Significant reduction was seen in } \\
\text { PD, CAL and BOP in the test } \\
\text { group as compared to control } \\
\text { group after } 6 \text { months }(p<0.05) \text {. } \\
\text { However, intergroup comparison } \\
\text { of PI showed nonsignificant } \\
\text { results }(p>0.05) \text {. Anaerobic } \\
\text { culture of plaque samples of test } \\
\text { group also revealed a significant } \\
\text { reduction of microorganisms in } \\
\text { comparison with control group. }\end{array}$ \\
\hline $\begin{array}{l}\text { Hokari et al., } \\
2018 \\
\text { (Japan) [79] }\end{array}$ & $\begin{array}{l}\text { International } \\
\text { Journal of } \\
\text { Dentistry } \\
\text { IF 2019: } 0.58 \\
\text { IF 2018: } 0.58\end{array}$ & PG-RCT & $\begin{array}{c}\mathrm{CP} \\
\text { (Moderate: } 3-4 \text { mm clinical } \\
\text { attachment loss, severe: } \geq 5 \mathrm{~mm} \\
\text { loss, generalized: }>30 \% \text { of } \\
\text { sites affected) }\end{array}$ & 30 & $\begin{array}{l}\text { aPDT } \\
\text { group: } 7 / 8 \\
\text { MO group: } \\
\quad 6 / 9\end{array}$ & $\begin{array}{l}\text { aPDT group: } \\
61.4 \pm 10.2 \\
\text { MO group: } \\
66.7 \pm 9.5\end{array}$ & $\begin{array}{l}\text { SRP+ Minocycline } \\
\text { ointment (MO) } \\
\text { (Ultrasonic } \\
\text { instruments) }\end{array}$ & SRP+aPDT & $\begin{array}{l}\text { Baseline, } 1 \\
\text { and } 4 \text { weeks }\end{array}$ & $\begin{array}{c}\text { BOP, PD, CAL, } \\
\text { PI, GI, } \\
\text { microbiological } \\
\text { and } \\
\text { inflammatory } \\
\text { marker analysis }\end{array}$ & $\begin{array}{l}\text { Local MO administration } \\
\text { exhibited a significant decrease in } \\
\text { scores for clinical parameters } \\
(p<0.01) \text { and a significant } \\
\text { reduction in bacterial counts } \\
(p<0.01) \text { and IL- } 1 \beta \text { and IF- } \gamma \\
\text { levels at } 1 \text { and } 4 \text { weeks after } \\
\text { treatment }(p<0.01) \text {. No significant } \\
\text { changes were observed in the } \\
\text { aPDT group, except in } \\
\text { clinical parameters. }\end{array}$ \\
\hline $\begin{array}{c}\text { Hill et al., } \\
2019 \\
\text { (Germany) } \\
{[80]}\end{array}$ & $\begin{array}{c}\text { Photodiagnosis } \\
\text { and } \\
\text { Photodynamic } \\
\text { Therapy } \\
\text { IF 2020: } 2.894 \\
\text { IF 2019: } 2.821\end{array}$ & SM-RCT & $\begin{array}{l}\mathrm{CP} \\
\text { (At least one single and one } \\
\text { multi-rooted tooth with at least } \\
4 \mathrm{~mm} \text { PPD in each quadrant) }\end{array}$ & 20 & $3 / 17$ & 61.1 & $\begin{array}{l}\text { SRP } \\
\text { (Hand and piezo- } \\
\text { electric ultrasonic } \\
\text { instruments) }\end{array}$ & $\mathrm{SRP}+\mathrm{aPDT}$ & $\begin{array}{l}\text { Baseline, } 2 \\
\text { week, } 3 \text { and } \\
6 \text { months }\end{array}$ & $\begin{array}{l}\text { BOP, SFFR, PD, } \\
\text { GR, RAL, } \\
\text { Microbiological } \\
\text { analysis }\end{array}$ & $\begin{array}{c}\text { Median values for BOP, RAL, PD, } \\
\text { decreased significantly in both } \\
\text { groups }(p<0.05) \text { after three } \\
\text { months of treatment without } \\
\text { significant difference between the } \\
\text { groups }(p>0.05) \text {. Two weeks after } \\
\text { treatment, the SFFR showed } \\
\text { significantly lower mean values in } \\
\text { the test group (aPDT). With the } \\
\text { applied parameters, this study } \\
\text { does not conclusively support } \\
\text { ICG-based aPDT, though it is } \\
\text { promising because no adverse } \\
\text { effects occurred. }\end{array}$ \\
\hline
\end{tabular}


Table 1. Cont.

\begin{tabular}{|c|c|c|c|c|c|c|c|c|c|c|c|c|c|}
\hline $\begin{array}{l}\text { Study, Year, } \\
\text { Origin and } \\
\text { Citation }\end{array}$ & $\begin{array}{l}\text { Journal Name/ } \\
\text { Impact Factor } \\
\text { (IF) }\end{array}$ & $\begin{array}{c}\text { Study } \\
\text { Design }\end{array}$ & Type of Periodontitis & $\begin{array}{l}\text { Sample } \\
\text { Size }(n)\end{array}$ & $\begin{array}{l}\text { Gender } \\
\mathrm{M} / \mathrm{F}\end{array}$ & $\begin{array}{c}\text { Age (Years) } \\
\text { (Mean } \pm \text { SD) }\end{array}$ & \multicolumn{4}{|c|}{ Intervention Groups } & $\begin{array}{l}\text { Evaluation } \\
\text { Period }\end{array}$ & $\begin{array}{l}\text { Parameters } \\
\text { Assessed }\end{array}$ & Conclusions \\
\hline $\begin{array}{c}\text { Ahad et al., } \\
2016 \\
\text { (India) [81] }\end{array}$ & $\begin{array}{l}\text { Journal of } \\
\text { Lasers in } \\
\text { Medical } \\
\text { Sciences } \\
\text { IF 2020: } 1.570 \\
\text { IF 2016: } 0.68\end{array}$ & SM-RCT & $\begin{array}{c}\mathrm{CP} \\
\text { (At least } 2 \text { teeth in different } \\
\text { quadrants with } \mathrm{PD} \geq 6 \mathrm{~mm} \text {, } \\
\text { and } \mathrm{BOP} \text { ) }\end{array}$ & 30 & $21 / 9$ & $38.67 \pm 10.52$ & \multicolumn{2}{|c|}{$\begin{array}{c}\text { SRP } \\
\text { (Hand and } \\
\text { ultrasonic } \\
\text { instruments) }\end{array}$} & \multicolumn{2}{|c|}{$\mathrm{SRP}+\mathrm{aPDT}$} & $\begin{array}{c}\text { Baseline, } 1 \\
\text { and } 3 \text { months }\end{array}$ & $\begin{array}{c}\text { PI, mSBI, PD, } \\
\text { CAL }\end{array}$ & $\begin{array}{l}\text { At } 1 \text { month follow-up, intergroup } \\
\text { difference in mean change was } \\
\text { statistically significant in terms of } \\
\text { mSBI and PD for the adjunctive } \\
\text { aPDT group ( } p<0.05) \text {, at } 3 \text { months } \\
\text { interval, no statistically significant } \\
\text { difference was observed between } \\
\text { test and control groups except in } \\
\text { terms of mSBI }(p>0.05) \text {, thus } \\
\text { proving that aPDT improved the } \\
\text { gingival status in the nonsurgical } \\
\text { management of CP. }\end{array}$ \\
\hline $\begin{array}{l}\text { Balata et al., } \\
2013 \\
\text { (Brazil) [82] }\end{array}$ & $\begin{array}{l}\text { Journal of } \\
\text { Applied Oral } \\
\text { Science } \\
\text { IF 2019: } 2.005 \\
\text { IF 2013: } 1.153\end{array}$ & SM-RCT & $\begin{array}{c}\text { CP } \\
\text { (Periodontal pockets with } \\
\text { CAL } \geq 5 \mathrm{~mm}, \mathrm{BOP} \text { and } \\
\text { radiographic bone loss; } \\
\text { minimum of } 2 \text { teeth with PD } \\
\geq 7 \mathrm{~mm} \text { and } 2 \text { other teeth } \\
\text { with a PD } \geq 5 \mathrm{~mm} \text {, all with } \\
\text { BOP and located on opposite } \\
\text { sides of the mouth; and } \geq 16 \\
\text { teeth in both jaws) }\end{array}$ & 22 & $8 / 14$ & 43.18 & \multicolumn{2}{|c|}{$\begin{array}{c}\text { SRP } \\
\text { (Ultrasonic } \\
\text { instruments) }\end{array}$} & \multicolumn{2}{|c|}{$\mathrm{SRP}+\mathrm{aPDT}$} & $\begin{array}{l}\text { Baseline, 1,3 } \\
\text { and } 6 \text { months }\end{array}$ & $\begin{array}{l}\text { PI, GI, BOP, GR, } \\
\text { CAL }\end{array}$ & $\begin{array}{l}\text { Both groups revealed statistically } \\
\text { significant improvement in the } \\
\text { clinical parameters }(p<0.05) \text { with } \\
\text { no statistically significant } \\
\text { differences upon intergroup } \\
\text { comparison }(p>0.05) \text {. aPDT did } \\
\text { not provide any additional benefit } \\
\text { to those obtained with full-mouth } \\
\text { ultrasonic debridement } \\
\text { used alone. }\end{array}$ \\
\hline
\end{tabular}


Table 1. Cont

\begin{tabular}{|c|c|c|c|c|c|c|c|c|c|c|c|}
\hline $\begin{array}{l}\text { Study, Year, } \\
\text { Origin and } \\
\text { Citation }\end{array}$ & $\begin{array}{l}\text { Journal Name/ } \\
\text { Impact Factor } \\
\text { (IF) }\end{array}$ & $\begin{array}{l}\text { Study } \\
\text { Design }\end{array}$ & Type of Periodontitis & $\begin{array}{l}\text { Sample } \\
\text { Size }(n)\end{array}$ & $\begin{array}{l}\text { Gender } \\
\mathbf{M} / \mathbf{F}\end{array}$ & $\begin{array}{c}\text { Age (Years) } \\
\text { (Mean } \pm \text { SD) }\end{array}$ & \multicolumn{2}{|c|}{ Intervention Groups } & $\begin{array}{l}\text { Evaluation } \\
\text { Period }\end{array}$ & $\begin{array}{l}\text { Parameters } \\
\text { Assessed }\end{array}$ & Conclusions \\
\hline $\begin{array}{l}\text { Bundidpun } \\
\text { et al., 2017 } \\
\text { (Thai- } \\
\text { land) [84] }\end{array}$ & $\begin{array}{l}\text { Laser Therapy } \\
\text { IF 2020: } 0.43 \\
\text { IF 2017: } 0.53\end{array}$ & SM-RCT & $\begin{array}{l}\text { CP } \\
\text { (Generalized moderate to severe } \\
\text { chronic periodontitis, presence } \\
\text { of at least } 20 \text { teeth, at least one } \\
\text { molar tooth in each quadrant } \\
\text { with a minimum of four teeth, at } \\
\text { least two teeth and one molar } \\
\text { tooth presented with PD }>6 \mathrm{~mm} \\
\text { in each quadrant) }\end{array}$ & 20 & $7 / 13$ & $47.25 \pm 8.91$ & $\begin{array}{c}\text { SRP } \\
\text { (Piezo-electric } \\
\text { ultrasonic } \\
\text { instruments) }\end{array}$ & $\mathrm{SRP}+\mathrm{aPDT}$ & $\begin{array}{l}\text { Baseline, 1, } 3 \\
\text { and } 6 \text { months }\end{array}$ & $\begin{array}{l}\text { PD, CAL, PI, } \\
\text { GBI, GI }\end{array}$ & $\begin{array}{l}\text { All parameters in test group were } \\
\text { better than that control group, } \\
\text { with statistically significant } \\
\text { differences of GBI and GI ( } p<0.05) \\
\text { at } 3 \text { and } 6 \text { months after treatment } \\
\text { but no statistically significant } \\
\text { differences of PD, CAL and PI. }\end{array}$ \\
\hline $\begin{array}{l}\text { Chitsazi et al., } \\
2014 \\
\text { (Iran) [86] }\end{array}$ & $\begin{array}{l}\text { Journal of } \\
\text { Advanced Pe- } \\
\text { riodontology } \\
\text { and Implant } \\
\text { Dentistry } \\
\text { IF 2020: NA } \\
\text { IF 2014: NA }\end{array}$ & SM-RCT & $\begin{array}{l}\mathrm{CP} \\
\text { (At least one site per quadrant } \\
\text { exhibiting pocket depth of } \geq 4 \\
\mathrm{~mm} \text { with bleeding on probing) }\end{array}$ & 22 & $10 / 12$ & 46.1 & $\begin{array}{c}\text { SRP } \\
\text { (Sonic instruments) }\end{array}$ & SRP+aPDT & $\begin{array}{l}\text { Baseline, } 1 \\
\text { and } 3 \text { months }\end{array}$ & $\begin{array}{l}\text { PD, CAL, BOP, } \\
\text { GR, } \\
\text { microbiological } \\
\text { analysis }\end{array}$ & $\begin{array}{l}\text { PD values decreased significantly } \\
\text { in both groups after } 1 \text { month } \\
(p=0.001) \text { and } 3 \text { months }(p=0.001) \\
\text { in the SRP and }(p=0.001) \text { in the } \\
\text { PDT groups the inter-group } \\
\text { differences were not significant } \\
\text { after } 1(p=0.25) \text { and } 3 \text { months } \\
(p=0.51) \text {. Clinical measurements } \\
\text { showed significant decreases after } \\
1 \text { and } 3 \text { months at both sites, } \\
\text { without inter-group differences, } \\
\text { except for BOP after } 1(p=0.004) \\
\text { and } 3 \text { months }(p=0.0001) .\end{array}$ \\
\hline $\begin{array}{l}\text { Garcia et al., } \\
2011 \\
\text { (Brazil) [87] }\end{array}$ & $\begin{array}{l}\text { Revista } \\
\text { Periodontia } \\
\text { IF 2020: NA } \\
\text { IF 2011: NA }\end{array}$ & SM-RCT & $\begin{array}{c}\mathrm{AgP} \\
\text { (Bone loss first molars and } \\
\text { incisors, and other teeth } \\
\text { adjacent, with PPD } \geq 5 \mathrm{~mm} \text { and } \\
\text { loss of CAL } \geq 2 \mathrm{~mm} \text { ) }\end{array}$ & 10 & $4 / 6$ & $39.3 \pm 5.84$ & $\begin{array}{c}\text { SRP } \\
\text { (Hand and ultrasonic } \\
\text { instruments) }\end{array}$ & SRP+aPDT & $\begin{array}{l}\text { Baseline, } 3 \\
\text { months }\end{array}$ & $\begin{array}{l}\text { PD, RCAL, } \\
\text { furcation } \\
\text { involvement, } \\
\text { tooth mobility }\end{array}$ & $\begin{array}{l}\text { Both groups showed improved } \\
\text { clinical results in the nonsurgical } \\
\text { treatment of AgP with no } \\
\text { statistically significant intergroup } \\
\text { differences }(p>0.05) \text {. }\end{array}$ \\
\hline
\end{tabular}


Table 1. Cont.

\begin{tabular}{|c|c|c|c|c|c|c|c|c|c|c|c|}
\hline $\begin{array}{l}\text { Study, Year, } \\
\text { Origin and } \\
\text { Citation }\end{array}$ & $\begin{array}{l}\text { Journal Name/ } \\
\text { Impact Factor } \\
\text { (IF) }\end{array}$ & $\begin{array}{c}\text { Study } \\
\text { Design }\end{array}$ & Type of Periodontitis & $\begin{array}{l}\text { Sample } \\
\text { Size }(n)\end{array}$ & $\begin{array}{l}\text { Gender } \\
\mathrm{M} / \mathrm{F}\end{array}$ & $\begin{array}{c}\text { Age (Years) } \\
(\text { Mean } \pm \text { SD) }\end{array}$ & \multicolumn{2}{|c|}{ Intervention Groups } & $\begin{array}{l}\text { Evaluation } \\
\text { Period }\end{array}$ & $\begin{array}{l}\text { Parameters } \\
\text { Assessed }\end{array}$ & Conclusions \\
\hline $\begin{array}{c}\text { Joseph et al., } \\
2014 \\
\text { (India) [88] }\end{array}$ & $\begin{array}{l}\text { Journal of } \\
\text { Clinical Peri- } \\
\text { odontology } \\
\text { IF 2020: } 5.241 \\
\text { IF 2014: } 4.641\end{array}$ & PG-RCT & $\begin{array}{c}\text { CP } \\
\text { (A minimum of } 20 \text { teeth; PPD } \\
4-6 \text { mm at least in two different } \\
\text { quadrants of the mouth) }\end{array}$ & 90 & $39 / 51$ & $39.6 \pm 8.7$ & $\begin{array}{c}\text { SRP } \\
\text { (Hand and ultrasonic } \\
\text { instruments) }\end{array}$ & $\mathrm{SRP}+\mathrm{aPDT}$ & $\begin{array}{l}\text { Baseline, } 2 \\
\text { weeks, } 1,3 \\
\text { and } 6 \text { months }\end{array}$ & $\begin{array}{l}\text { PPD, CAL, GI, } \\
\text { GBI, PI, } \\
\text { halitosis. }\end{array}$ & $\begin{array}{l}\text { PD and CAL showed statistically } \\
\text { significant reduction in the test } \\
\text { group on evaluation at } 3 \text { months } \\
\text { and } 6 \text { months as compared to the } \\
\text { control group }(p<0.05) \text {. A } \\
\text { statistically significant } \\
\text { improvement in GI and GBI was } \\
\text { seen for the test group after } \\
2 \text { weeks and } 1 \text { month of aPDT } \\
(p<0.01) \text {, whereas the } \\
\text { improvement in GI and GBI at } \\
3 \text { months and in plaque index at } \\
2 \text { weeks after aPDT was less } \\
\text { ( } p<0.05) \text {. In addition, a significant } \\
\text { difference was detected for the test } \\
\text { group at } 1 \text { month in terms of } \\
\text { halitosis, which did not persist for } \\
\text { long }(p<0.05) \text {. }\end{array}$ \\
\hline $\begin{array}{l}\text { Malgikar } \\
\text { et al., 2015 } \\
\text { (India) [89] }\end{array}$ & $\begin{array}{l}\text { Journal of } \\
\text { Dental Lasers } \\
\text { IF 2020: 0.696 } \\
\text { IF 2015: NA }\end{array}$ & SM-RCT & $\begin{array}{c}\mathrm{CP} \\
\text { (At least one site in each } \\
\text { quadrant of the mouth having } \\
\text { deep PPD } \geq 5 \mathrm{~mm} \text { and } \\
\text { radiographic signs of alveolar } \\
\text { bone loss) }\end{array}$ & 24 & $15 / 9$ & $\begin{array}{c}\text { M: } 36.73 \pm \\
8.46 \\
\text { F: } 34.33 \pm 6.80\end{array}$ & $\begin{array}{l}\text { SRP } \\
\text { (Hand and piezo- } \\
\text { electric ultrasonic } \\
\text { instruments) }\end{array}$ & $\mathrm{SRP}+\mathrm{aPDT}$ & $\begin{array}{l}\text { Baseline, 1,3 } \\
\text { and 6 } \\
\text { months. }\end{array}$ & $\begin{array}{l}\text { PI, GI, mSBI, } \\
\text { PD, CAL. }\end{array}$ & $\begin{array}{l}\text { A statistically significant decrease } \\
\text { in PD, CAL, PI, GI, mSBI scores } \\
\text { was seen in SRP+aPDT at the end } \\
\text { of } 6 \text { months }(p<0.001) .\end{array}$ \\
\hline $\begin{array}{l}\text { Monzavi } \\
\text { et al., 2016 } \\
\text { (Iran) [90] }\end{array}$ & 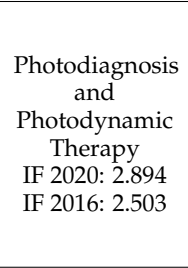 & SM-RCT & $\begin{array}{l}\mathrm{CP} \\
\text { (At least three teeth exhibiting } \\
\text { residual pocket depth of } \\
\geq 5 \mathrm{~mm} \text { with bleeding } \\
\text { on probing) }\end{array}$ & 50 & $25 / 25$ & $49.6 \pm 8.5$ & $\begin{array}{c}\text { SRP } \\
\text { (Hand and ultrasonic } \\
\text { instruments) }\end{array}$ & $\mathrm{SRP}+\mathrm{aPDT}$ & $\begin{array}{l}\text { Baseline, } 1 \\
\text { and } 3 \text { months }\end{array}$ & $\begin{array}{l}\text { BOP, PI, CAL, } \\
\text { PPD, FMPS, } \\
\text { FMBS }\end{array}$ & $\begin{array}{l}\text { There were no significant } \\
\text { differences between two groups at } \\
\text { baseline. BOP, PPD and FMBS } \\
\text { showed significant improvements } \\
\text { in the test group ( } p \leq 0.001) \text {. In } \\
\text { terms of PI, FMPS and CAL, no } \\
\text { significant differences were } \\
\text { observed between both groups } \\
(p \geq 0.05) \text {. }\end{array}$ \\
\hline $\begin{array}{l}\text { Raj et al., } \\
2016 \\
\text { (India) [91] }\end{array}$ & $\begin{array}{l}\text { Indian Journal } \\
\text { of Dental } \\
\text { Research } \\
\text { IF 2020: } 0.37 \\
\text { IF 2016: } 0.08\end{array}$ & PG-RCT & $\begin{array}{c}\mathrm{CP} \\
\text { (More than } 16 \text { natural teeth; } \\
\operatorname{PPD} \geq 5 \mathrm{~mm} \text { ) }\end{array}$ & 20 & $8 / 12$ & NI & $\begin{array}{c}\text { SRP } \\
\begin{array}{c}\text { (Type of instruments } \\
\text { utilized-NI) }\end{array}\end{array}$ & $\mathrm{SRP}+\mathrm{aPDT}$ & $\begin{array}{l}\text { Baseline and } \\
3 \text { months }\end{array}$ & $\begin{array}{l}\text { PI, GI, PD, CAL } \\
\text { and } \\
\text { microbiological } \\
\text { analysis }\end{array}$ & $\begin{array}{l}\text { There was a significant reduction } \\
\text { in PI, GI, PD, CAL and } \\
\text { microbiologic parameters in test } \\
\text { group, following SRP and PDT, } \\
\text { when compared with SRP alone in } \\
\text { control group ( } p<0.001) \text {. } \\
\text { SRP+aPDT has shown additional } \\
\text { improvement in periodontal } \\
\text { parameters when compared to SRI } \\
\text { alone and has a beneficial effect in } \\
\text { chronic periodontitis patients. }\end{array}$ \\
\hline
\end{tabular}


Table 1. Cont

\begin{tabular}{|c|c|c|c|c|c|c|c|c|c|c|c|}
\hline $\begin{array}{l}\text { Study, Year, } \\
\text { Origin and } \\
\text { Citation }\end{array}$ & $\begin{array}{l}\text { Journal Name/ } \\
\text { Impact Factor } \\
\text { (IF) }\end{array}$ & $\begin{array}{c}\text { Study } \\
\text { Design }\end{array}$ & Type of Periodontitis & $\begin{array}{l}\text { Sample } \\
\text { Size }(n)\end{array}$ & $\begin{array}{l}\text { Gender } \\
\mathbf{M} / \mathrm{F}\end{array}$ & $\begin{array}{c}\text { Age (Years) } \\
\text { (Mean } \pm \text { SD) }\end{array}$ & \multicolumn{2}{|c|}{ Intervention Groups } & $\begin{array}{l}\text { Evaluation } \\
\text { Period }\end{array}$ & $\begin{array}{l}\text { Parameters } \\
\text { Assessed }\end{array}$ & Conclusions \\
\hline $\begin{array}{l}\text { Sena et al., } \\
2019 \\
\text { (Brazil) [92] }\end{array}$ & $\begin{array}{l}\text { Photobiomo- } \\
\text { dulation, } \\
\text { Photomedicine } \\
\text { and Laser } \\
\text { Surgery } \\
\text { IF 2019: } 1.913\end{array}$ & SM-RCT & $\begin{array}{c}\text { CP } \\
\text { (At least six sites with PD } \\
\text { 5-9 mm; and BOP) }\end{array}$ & $\begin{array}{l}9 \text { (6 sites / } \\
\text { patient: } \\
\text { total-54 } \\
\text { sites) }\end{array}$ & NI & NI & $\begin{array}{c}\text { SRP+ placebo } \\
\text { procedure } \\
\text { (Hand and ultrasonic } \\
\text { instruments) }\end{array}$ & $\mathrm{SRP}+\mathrm{aPDT}$ & $\begin{array}{l}\text { Baseline and } \\
3 \text { months }\end{array}$ & $\begin{array}{l}\text { BOP, PD, CAL, } \\
\text { VPI }\end{array}$ & $\begin{array}{c}\text { There was a statistically significant } \\
\text { decrease in BOP for test group } \\
(p=0.003) \text { and control group } \\
(p=0.001) \text {. Intragroup comparison } \\
\text { for PD and CAL showed } \\
\text { statistically significant differences } \\
\text { from baseline }(p<0.05) \text { with no } \\
\text { intergroup differences }(p>0.05) \text {. } \\
\text { Hence, SRP+aPDT did not show } \\
\text { any additional benefits over } \\
\text { SRP alone. }\end{array}$ \\
\hline $\begin{array}{l}\text { Shingnapurkar } \\
\text { et al., 2016 } \\
\text { (India) [93] }\end{array}$ & $\begin{array}{l}\text { Indian Journal } \\
\text { of Dental } \\
\text { Research } \\
\text { IF 2020: } 0.37 \\
\text { IF 2016: } 0.08\end{array}$ & SM-RCT & $\begin{array}{c}\mathrm{CP} \\
(\mathrm{PD}>5 \mathrm{~mm})\end{array}$ & 60 sites & NI & NI & $\begin{array}{l}\mathrm{SRP}+\text { sham procedure } \\
\text { (Hand and ultrasonic } \\
\text { instruments) }\end{array}$ & $\mathrm{SRP}+\mathrm{aPDT}$ & $\begin{array}{c}\text { Baseline, } 1 \\
\text { and } 3 \text { months }\end{array}$ & PI, GI, PD, RAL & $\begin{array}{l}\text { Mean baseline values for PI, GI, } \\
\text { PPD and RAL were not different in } \\
\text { the test group and control group. } \\
\text { Statistically significant difference } \\
\text { in PPD and RAL, } 3 \text { months after } \\
\text { treatment was seen in test group } \\
\text { as compared to the control group } \\
\text { ( } p<0.05) .\end{array}$ \\
\hline $\begin{array}{l}\text { Sigusch et al., } \\
2010 \text { (Ger- } \\
\text { many) [94] }\end{array}$ & $\begin{array}{l}\text { Journal of Peri- } \\
\text { odontology } \\
\text { IF 2020: } 3.742 \\
\text { IF 2010: } 2.946\end{array}$ & PG-RCT & $\begin{array}{c}\mathrm{CP} \\
(<30 \% \text { of sites with } \\
\text { PPD }>3.5 \mathrm{~mm})\end{array}$ & $\begin{array}{l}24 \text { (12 in } \\
\text { each } \\
\text { group) }\end{array}$ & $\begin{array}{l}\text { PDT group: } \\
4 / 8 \\
\text { Control } \\
\text { group: } 3 / 9\end{array}$ & $\begin{array}{l}\text { PDT group } \\
\text { F: } 39.75 \\
\text { M: } 45 \\
\text { Control group: } \\
\text { F: } 44.22 \\
\text { M: } 42.67\end{array}$ & $\begin{array}{l}\text { SRP+ sham procedure } \\
\text { (Type of instruments } \\
\text { utilized- NI) }\end{array}$ & $\mathrm{SRP}+\mathrm{aPDT}$ & $\begin{array}{l}\text { Baseline, 1, } 4 \\
\quad \text { and } 12 \\
\text { weeks. }\end{array}$ & $\begin{array}{c}\text { PI, reddening, } \\
\text { PD, BOP, CAL, } \\
\text { GR } \\
\text { Quantitative } \\
\text { analysis for } F . n .\end{array}$ & $\begin{array}{l}\text { In patients with localized CP who } \\
\text { received aPDT treatment, } \\
\text { significant reductions in } \\
\text { reddening, BOP, and mean PD and } \\
\text { CAL were observed during the } \\
\text { observation period and with } \\
\text { respect to controls }(p<0.001) \text {. Four } \\
\text { and } 12 \text { weeks after aPDT, the mean } \\
\text { PD and CAL showed significant } \\
\text { differences from baseline values } \\
\text { and from those of the control } \\
\text { group. In the aPDT group, } \\
12 \text { weeks after treatment, the } F . n \text {. } \\
\text { DNA concentration was found to } \\
\text { be significantly reduced compared } \\
\text { to the baseline level ( } p<0.001) \\
\text { compared to control group. }\end{array}$ \\
\hline
\end{tabular}


Table 1. Cont

\begin{tabular}{|c|c|c|c|c|c|c|c|c|c|c|c|c|}
\hline $\begin{array}{l}\text { Study, Year, } \\
\text { Origin and } \\
\text { Citation }\end{array}$ & $\begin{array}{l}\text { Journal Name/ } \\
\text { Impact Factor } \\
\text { (IF) }\end{array}$ & $\begin{array}{c}\text { Study } \\
\text { Design }\end{array}$ & Type of Periodontitis & $\begin{array}{l}\text { Sample } \\
\text { Size }(n)\end{array}$ & $\begin{array}{l}\text { Gender } \\
\text { M/F }\end{array}$ & $\begin{array}{c}\text { Age (Years) } \\
\text { (Mean } \pm \text { SD) }\end{array}$ & \multicolumn{3}{|c|}{ Intervention Groups } & $\begin{array}{l}\text { Evaluation } \\
\text { Period }\end{array}$ & $\begin{array}{l}\text { Parameters } \\
\text { Assessed }\end{array}$ & Conclusions \\
\hline $\begin{array}{l}\text { Theodoro } \\
\text { et al., 2012 } \\
\text { (Brazil) [95] }\end{array}$ & $\begin{array}{l}\text { Lasers in } \\
\text { Medical } \\
\text { Science } \\
\text { IF 2019: } 2.574 \\
\text { IF 2012: } 2.645\end{array}$ & SM-RCT & $\begin{array}{c}\mathrm{CP} \\
\text { (At least three non-adjacent } \\
\text { sites with BOP and a PD of } \\
5-9 \text { mm at least } 20 \text { teeth in } \\
\text { the oral cavity) }\end{array}$ & 33 & $12 / 21$ & $43.12 \pm 8.2$ & $\begin{array}{c}\text { SRP } \\
\text { (Hand instruments) }\end{array}$ & $\begin{array}{l}\text { SRP+ } \\
\text { PS } \\
\text { (TBO) } \\
\text { only }\end{array}$ & $\begin{array}{l}\text { SRP+ } \\
\text { aPDT }\end{array}$ & $\begin{array}{l}\text { Baseline, } 60, \\
90 \text { and } \\
180 \text { days }\end{array}$ & $\begin{array}{c}\text { VPI, GI, BOP, } \\
\text { PD, CAL, GR, } \\
\text { microbiological } \\
\text { analysis }\end{array}$ & $\begin{array}{l}\text { All treatment groups showed an } \\
\text { improvement in all clinical } \\
\text { parameters, and a significant } \\
\text { reduction in the proportion of sites } \\
\text { positive for periodontopathogens } \\
\text { at } 60,90 \text { and } 180 \text { days compared to } \\
\text { baseline }(p<0.05) \text {. None of the } \\
\text { periodontal parameters showed a } \\
\text { significant difference among the } \\
\text { groups }(p>0.05) \text {. At } 180 \text { days, } \\
\text { PDT treatment led to a significant } \\
\text { reduction in the percentage of sites } \\
\text { positive for all bacteria compared } \\
\text { to SRP alone }(p<0.05) \text {. }\end{array}$ \\
\hline $\begin{array}{l}\text { Theodoro } \\
\text { et al., 2017 } \\
\text { (Brazil) [96] }\end{array}$ & $\begin{array}{c}\text { Journal of Pho- } \\
\text { tochemistry } \\
\text { and } \\
\text { Photobiology } \\
\text { B } \\
\text { IF 2020: } 4.383 \\
\text { IF 2017: } 3.438\end{array}$ & PG-RCT & $\begin{array}{c}\mathrm{CP} \\
\text { (Severe generalized CP in at } \\
\text { least } 6 \text { teeth and with one or } \\
\text { several sites with } \\
\mathrm{PD} \geq 5 \mathrm{~mm} \text {; a loss of } \\
\text { CAL } \geq 5 \mathrm{~mm} \text {; a minimum of } \\
30 \% \text { of the sites with PD and } \\
\mathrm{CAL} \geq 4 \mathrm{~mm} \text { and BOP; and } \\
\text { the presence of at least } \\
15 \text { teeth) }\end{array}$ & 34 & $\begin{array}{l}\text { AB group: } \\
7 / 7 \\
\text { aPDT } \\
\text { group: } 9 / 5\end{array}$ & $\begin{array}{l}\text { AB group: } \\
\text { 46.3 } \pm 6.8 \\
\text { aPDT group: } \\
48.8 \pm 8.3\end{array}$ & $\begin{array}{c}\text { SRP+ (MTZ+ AMX) } \\
\text { MTZ dose: } 400 \mathrm{mg} \\
\text { TDS-7 days } \\
\text { AMX dose: } 500 \mathrm{mg} \\
\text { TDS-7 days } \\
\text { (Type of instruments } \\
\text { utilized for SRP-NI) }\end{array}$ & $\begin{array}{l}\text { SRP +a } \\
\text { placebc }\end{array}$ & $\begin{array}{l}\text { PDT+ } \\
\text { o pills }\end{array}$ & $\begin{array}{l}\text { Baseline and } \\
90 \text { days }\end{array}$ & $\mathrm{BOP}, \mathrm{PD}, \mathrm{CAL}$ & $\begin{array}{l}\text { There was a significant } \\
\text { improvement in CAL only in the } \\
\text { intermediate pocket in the aPDT } \\
\text { group com- pared to the } \\
\text { MTZ + AMX group between } \\
\text { baseline and } 90 \text { days } \\
\text { post-treatment }(p=0.01) \text {. There } \\
\text { was a reduction of both BOP and } \\
\text { the percentage of residual pockets } \\
\text { at } 90 \text { days after treatment } \\
\text { compared with baseline in both } \\
\text { groups }(p<0.05) \text {. }\end{array}$ \\
\hline
\end{tabular}




\subsubsection{Documentation of Laser Parameters}

Table 2 describes various dye laser combinations, as well as laser dosimetry that was utilized to perform aPDT in all eligible studies. Twenty-six out of 31 studies utilized a laser wavelength in the range of 630-690 $\mathrm{nm}[2,3,5,17,70-77,79,81-88,91,92,94-96]$ to perform aPDT. While four studies utilized a laser wavelength in the range of 808-810 $\mathrm{nm}[78,80,90,93]$, one of the included studies utilized a $980 \mathrm{~nm}$ diode laser wavelength to perform aPDT [89] (Table 2) (Figure 2). Emission mode was reported only in five studies $[78,88,90,92,93]$, in which four of them utilized a continuous wave emission mode $[78,88,90,92]$, whilst the remaining one study utilized a gated continuous wave emission mode [93]. Eighteen out of the 31 eligible studies used the laser fibre tip in 'contact mode' with the periodontal pocket in order to perform aPDT $[2,3,5,78,81,82,84-86,88-96]$. Only 5 studies reported total energy, and it ranged from $1.5-9 \mathrm{~J}[82,90,92,93,95,96]$. Only 19 studies reported power output in the range of $30 \mathrm{~mW}-1 \mathrm{~W}[71,75-80,82-85,87,89-96]$, whereas the use of a power meter to measure the therapeutic power output, reaching the target tissues was not performed in any of the included studies. Spot size was reported in only four studies $[5,85,92,96]$ ranging from $0.02-0.07 \mathrm{~cm}^{2}$. Ten out of the 31 studies reported the diameter of fibre tip, $[2,3,5,80-82,88,89,93,94]$ ranging from $200-600 \mu \mathrm{m}$. The energy density (fluence) was calculated in 18 out of 31 studies $[5,17,70-74,78-80,82,83,86,87,92,93,95,96]$, and its value ranged from $0.01-2829 \mathrm{~J} / \mathrm{cm}^{2}$, whereas the power density (irradiance) values ranged from $60 \mathrm{~mW}-4 \mathrm{~W} / \mathrm{cm}^{2}$ and were calculated in 13 studies [2,3,5,17,71-74,81,88,92,94,95]. Finally, the exposure time for laser irradiation was mentioned in all included studies except one study [80], and the values ranged from 10-120 s/site amongst included studies.

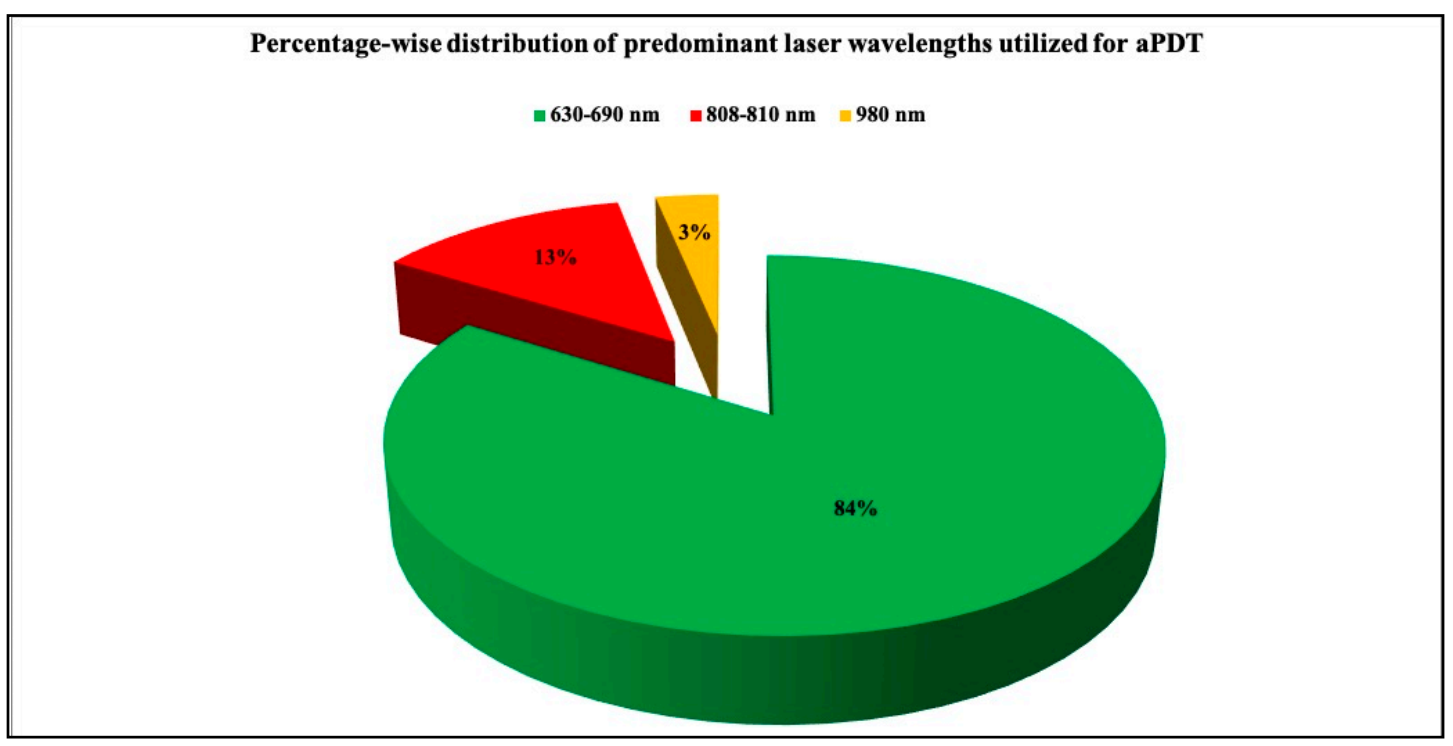

Figure 2. 3D pie diagram illustrating the percentage-wise distribution of predominant laser wavelengths utilized for aPDT in the included studies.

\subsubsection{PS Utilized}

Type of PS varied amongst eligible clinical trials. Eleven studies utilized phenothiazine chloride $[2,3,5,71-74,76,81,84,94]$ while 10 employed methylene blue $[17,75,77,79,82,87-$ $89,96]$. Five studies utilized toluidine blue O $[70,85,86,91,95]$, four studies used indocyanine green $[78,80,90,93]$, whereas chloro-aluminum phthalocyanine was utilized in one study [92] (Figure 3). Interestingly, 18 out of 31 studies specified the concentration of the PS $[2,3,17,71,75,77-80,82,83,87-90,92,95,96]$, while 13 studies failed to report the same $[5,70,72-74,76,81,84-86,91,93,94]$ (Table 2). 


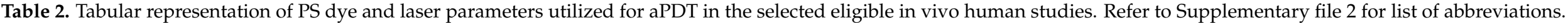

\begin{tabular}{|c|c|c|c|c|c|c|c|c|c|c|c|c|c|c|}
\hline $\begin{array}{l}\text { Study, Year, } \\
\text { Origin and } \\
\text { Citation }\end{array}$ & $\begin{array}{l}\text { Photosensi- } \\
\text { tizer (PS) } \\
\text { Used and } \\
\text { Its Concen- } \\
\text { tration }\end{array}$ & $\begin{array}{c}\text { Pre- } \\
\text { Irradiation } \\
\text { Exposure } \\
\text { Time to PS } \\
\text { (min) }\end{array}$ & $\begin{array}{c}\text { Laser } \\
\text { Wavelength } \\
\text { Utilized }\end{array}$ & $\begin{array}{l}\text { Emission Mode } \\
\text { Contact/No } \\
\text { Contact } \\
\text { Tip Initiation }\end{array}$ & $\begin{array}{c}\text { Energy } \\
\text { (J) }\end{array}$ & $\begin{array}{l}\text { Power } \\
\text { Output } \\
\text { (W) }\end{array}$ & $\begin{array}{c}\text { Pulse } \\
\text { Length } \\
\text { (Duration), } \\
\text { Pulse } \\
\text { Interval }\end{array}$ & $\begin{array}{l}\text { Use of } \\
\text { Power } \\
\text { Meter }\end{array}$ & $\begin{array}{c}\text { Distance } \\
\text { from Target }\end{array}$ & $\begin{array}{c}\text { Spot } \\
\text { Size/Fibre-Tip } \\
\text { Diame- } \\
\text { ter/Spot } \\
\text { Diameter }\end{array}$ & $\begin{array}{l}\text { Energy } \\
\text { Density } \\
{[\text { Fluence] }} \\
\left(\mathrm{J} / \mathrm{cm}^{2}\right)\end{array}$ & $\begin{array}{c}\text { Power } \\
\text { Density } \\
\text { [Irradiance] } \\
\left(\mathrm{W} / \mathrm{cm}^{2}\right)\end{array}$ & $\begin{array}{c}\text { Exposure Time } \\
\text { to Laser } \\
\text { Irradiation } \\
\text { [Minute }(\min ) / \\
\text { Second (s)] }\end{array}$ & $\begin{array}{l}\text { No. of } \\
\text { aPDT Ap- } \\
\text { plications }\end{array}$ \\
\hline $\begin{array}{l}\text { De Oliveira } \\
\text { et al., 2009 } \\
\text { (Brazil) [2] }\end{array}$ & $\begin{array}{l}\text { Phenothiazine } \\
\text { chloride } \\
(10 \mathrm{mg} / \mathrm{mL})\end{array}$ & $1 \mathrm{~min}$ & $660 \mathrm{~nm}$ & $\begin{array}{l}\text { Contact mode, fibre } \\
\text { tip was place at the } \\
\text { entrance of the } \\
\text { gingival sulcus }\end{array}$ & NI & $\mathrm{NI}$ & NI & NI & NA & $\begin{array}{l}\text { Tip diameter: } \\
600 \mu \mathrm{m}\end{array}$ & NI & $60 \mathrm{~mW} / \mathrm{cm}^{2}$ & $\begin{array}{l}10 \mathrm{~s} / \mathrm{site} \\
(6 \mathrm{sites}=1 \\
\mathrm{min} / \text { tooth })\end{array}$ & 1 \\
\hline $\begin{array}{l}\text { De Oliveira } \\
\text { et al., 2007 } \\
\text { (Brazil) [3] }\end{array}$ & $\begin{array}{l}\text { Phenothiazine } \\
\text { chloride } \\
(10 \mathrm{mg} / \mathrm{mL})\end{array}$ & $1 \mathrm{~min}$ & $660 \mathrm{~nm}$ & $\begin{array}{l}\text { Contact mode, fibre } \\
\text { tip was place at the } \\
\text { entrance of the } \\
\text { gingival sulcus }\end{array}$ & NI & NI & NI & NI & NI & $\begin{array}{l}\text { Tip diameter: } \\
600 \mu \mathrm{m}\end{array}$ & NI & $60 \mathrm{~mW} / \mathrm{cm}^{2}$ & $\begin{array}{l}10 \mathrm{~s} / \mathrm{site} \\
(6 \mathrm{sites}=1 \\
\mathrm{min} / \text { tooth })\end{array}$ & 1 \\
\hline $\begin{array}{c}\text { Novaes } \\
\text { et al., 2012 } \\
\text { (Brazil) [5] }\end{array}$ & $\begin{array}{l}\text { Phenothiazine } \\
\text { chloride }\end{array}$ & NI & $660 \mathrm{~nm}$ & $\begin{array}{l}\text { Contact mode, fibre } \\
\text { tip was place at the } \\
\text { entrance of the } \\
\text { gingival sulcus }\end{array}$ & $\mathrm{NI}$ & NI & NI & NI & NI & $\begin{array}{l}\text { Tip diameter: } \\
600 \mu \mathrm{m} \\
\text { [8.5 cm long } \\
\text { optic fibre } \\
\text { with } 60^{\circ} \\
\text { angulated tip] } \\
\text { Spot size: } 0.06\end{array}$ & $\begin{array}{l}212.23 \\
\mathrm{~J} / \mathrm{cm}^{2}\end{array}$ & $60 \mathrm{~mW} / \mathrm{cm}^{2}$ & $\begin{array}{l}10 \mathrm{~s} / \mathrm{site} \\
(6 \mathrm{sites} / \text { tooth }) \\
60 \mathrm{~s} / \text { tooth }\end{array}$ & 1 \\
\hline $\begin{array}{c}\text { Franco et al., } \\
2014 \\
\text { (Brazil) [17] }\end{array}$ & $\begin{array}{l}\text { Methylene } \\
\text { blue }(0.01 \%)\end{array}$ & $5 \mathrm{~min}$ & $660 \mathrm{~nm}$ & NI & NI & NI & NI & NI & NI & NI & $5.4 \mathrm{~J} / \mathrm{cm}^{2}$ & $60 \mathrm{~mW} / \mathrm{cm}^{2}$ & $\begin{array}{c}5 \mathrm{~s} / \mathrm{site} \\
(6 \mathrm{sites} / \text { tooth }) \\
90 \mathrm{~s} / \text { tooth }\end{array}$ & 4 \\
\hline $\begin{array}{l}\text { Moreira } \\
\text { et al., 2015 } \\
\text { (Brazil) [71] }\end{array}$ & $\begin{array}{l}\text { Phenothiazine } \\
\text { chloride } \\
(10 \mathrm{mg} / \mathrm{mL})\end{array}$ & $1 \mathrm{~min}$ & $670 \mathrm{~nm}$ & NI & NI & $75 \mathrm{~mW}$ & NI & NI & NI & $\begin{array}{l}\text { Tip diameter: } \\
600 \mu \mathrm{m}\end{array}$ & $\begin{array}{l}\text { Fluence } / \text { site: } \\
2.49 \mathrm{~J} / \mathrm{cm}^{2} \\
\text { Fluence } / \text { tooth: } \\
14.94 \mathrm{~J} / \mathrm{cm}^{2}\end{array}$ & $0.25 \mathrm{~W} / \mathrm{cm}^{2}$ & $10 \mathrm{~s} / \mathrm{site}$ & $\begin{array}{c}4 \\
(0,2 \text { nd, } 7 \text { th } \\
\text { and } \\
\text { 14th day })\end{array}$ \\
\hline $\begin{array}{c}\text { Skurska } \\
\text { et al., 2015 } \\
\text { (Poland) } \\
\text { [72] }\end{array}$ & $\begin{array}{l}\text { Phenothiazine } \\
\text { chloride }\end{array}$ & $3 \mathrm{~min}$ & $660 \mathrm{~nm}$ & NI & NI & NI & NI & NI & NI & NI & $120 \mathrm{~J} / \mathrm{cm}^{2}$ & $60 \mathrm{mw} / \mathrm{cm}^{2}$ & $60 \mathrm{~s} / \mathrm{site}$ & $\begin{array}{c}2 \\
(0 \text { and } \\
7 \text { th day) }\end{array}$ \\
\hline $\begin{array}{c}\text { Arweiler } \\
\text { et al., 2014 } \\
\text { (Poland) [73] }\end{array}$ & $\begin{array}{l}\text { Phenothiazine } \\
\text { chloride }\end{array}$ & $3 \min$ & $660 \mathrm{~nm}$ & NI & NI & $\mathrm{NI}$ & NI & NI & NI & NI & $120 \mathrm{~J} / \mathrm{cm}^{2}$ & $60 \mathrm{mw} / \mathrm{cm}^{2}$ & $60 \mathrm{~s} / \mathrm{site}$ & $\begin{array}{c}2 \\
(0 \text { and } \\
7 \text { th day) }\end{array}$ \\
\hline $\begin{array}{c}\text { Arweiler } \\
\text { et al., 2013 } \\
\text { (Poland) [74] }\end{array}$ & $\begin{array}{l}\text { Phenothiazine } \\
\text { chloride }\end{array}$ & $3 \mathrm{~min}$ & $660 \mathrm{~nm}$ & NI & NI & $\mathrm{NI}$ & NI & NI & NI & $\mathrm{NI}$ & $120 \mathrm{~J} / \mathrm{cm}^{2}$ & $60 \mathrm{mw} / \mathrm{cm}^{2}$ & $60 \mathrm{~s} / \mathrm{site}$ & $\begin{array}{c}2 \\
(0 \text { and } \\
7 \text { th day })\end{array}$ \\
\hline
\end{tabular}


Table 2. Cont.

\begin{tabular}{|c|c|c|c|c|c|c|c|c|c|c|c|c|c|c|}
\hline $\begin{array}{l}\text { Study, Year, } \\
\text { Origin and } \\
\text { Citation }\end{array}$ & $\begin{array}{l}\text { Photosensi- } \\
\text { tizer (PS) } \\
\text { Used and } \\
\text { Its Concen- } \\
\text { tration }\end{array}$ & $\begin{array}{c}\text { Pre- } \\
\text { Irradiation } \\
\text { Exposure } \\
\text { Time to PS } \\
\text { (min) }\end{array}$ & $\begin{array}{c}\text { Laser } \\
\text { Wavelength } \\
\text { Utilized }\end{array}$ & $\begin{array}{l}\text { Emission Mode } \\
\text { Contact/No } \\
\text { Contact } \\
\text { Tip Initiation }\end{array}$ & $\begin{array}{c}\text { Energy } \\
\text { (J) }\end{array}$ & $\begin{array}{l}\text { Power } \\
\text { Output } \\
\text { (W) }\end{array}$ & $\begin{array}{c}\text { Pulse } \\
\text { Length } \\
\text { (Duration), } \\
\text { Pulse } \\
\text { Interval }\end{array}$ & $\begin{array}{l}\text { Use of } \\
\text { Power } \\
\text { Meter }\end{array}$ & $\begin{array}{c}\text { Distance } \\
\text { from Target }\end{array}$ & $\begin{array}{c}\text { Spot } \\
\text { Size/Fibre-Tip } \\
\text { Diame- } \\
\text { ter/Spot } \\
\text { Diameter } \\
\end{array}$ & $\begin{array}{c}\text { Energy } \\
\text { Density } \\
\text { [Fluence] } \\
\left(\mathrm{J} / \mathrm{cm}^{2}\right)\end{array}$ & $\begin{array}{c}\text { Power } \\
\text { Density } \\
\text { [Irradiance] } \\
\left(\mathrm{W} / \mathrm{cm}^{2}\right)\end{array}$ & $\begin{array}{c}\text { Exposure Time } \\
\text { to Laser } \\
\text { Irradiation } \\
\text { [Minute (min)/ } \\
\text { Second (s)] }\end{array}$ & $\begin{array}{c}\text { No. of } \\
\text { aPDT Ap- } \\
\text { plications }\end{array}$ \\
\hline $\begin{array}{l}\text { Vidal et al., } \\
2017 \\
\text { (Spain) [75] }\end{array}$ & $\begin{array}{l}\text { Methylene } \\
\text { blue } \\
(0.005 \%)\end{array}$ & NI & $670 \mathrm{~nm}$ & NI & NI & $150 \mathrm{~mW}$ & NI & NI & NI & NI & NI & NI & $60 \mathrm{~s} /$ pocket & $\begin{array}{c}3 \\
\text { (1,5 and } \\
13 \text { weeks) }\end{array}$ \\
\hline $\begin{array}{c}\text { Braun et al., } \\
2008 \\
\text { (Germany) } \\
{[76]}\end{array}$ & $\begin{array}{l}\text { Phenothiazine } \\
\text { chloride }\end{array}$ & $3 \mathrm{~min}$ & $660 \mathrm{~nm}$ & NI & NI & $100 \mathrm{~mW}$ & NI & NI & NI & NI & $\mathrm{NI}$ & NI & $\begin{array}{c}10 \mathrm{~s} / \mathrm{site} \\
(6 \mathrm{sites}=1 \mathrm{~min} \\
/ \text { tooth })\end{array}$ & 1 \\
\hline $\begin{array}{c}\text { Berakdar } \\
\text { et al., } 2012 \\
\text { (Germany) } \\
{[77]}\end{array}$ & $\begin{array}{c}\text { Methylene } \\
\text { blue } \\
0.005 \%\end{array}$ & NI & $670 \mathrm{~nm}$ & NI & NI & $150 \mathrm{~mW}$ & NI & NI & NI & NI & $\mathrm{NI}$ & NI & $1 \mathrm{~min}$ & 1 \\
\hline $\begin{array}{c}\text { Raut et al., } \\
2018 \text { (India) } \\
\text { [78] }\end{array}$ & $\begin{array}{c}\text { Indocyanine } \\
\text { green } \\
(5 \mathrm{mg} / \mathrm{mL})\end{array}$ & $60 \mathrm{~s}$ & $810 \mathrm{~nm}$ & $\mathrm{CW}$, contact mode & NI & $80 \mathrm{~mW}$ & NI & NI & NA & NI & $5.4 \mathrm{~J} / \mathrm{cm}^{2}$ & NI & $60 \mathrm{~s}$ & 1 \\
\hline $\begin{array}{c}\text { Hokari et al., } \\
2018 \text { (Japan) } \\
\text { [79] }\end{array}$ & $\begin{array}{c}\text { Methylene } \\
\text { blue dye } \\
0.01 \%\end{array}$ & $1 \mathrm{~min}$ & $670 \mathrm{~nm}$ & NI, contact mode & NI & $140 \mathrm{~mW}$ & NI & NI & NA & NI & $21 \mathrm{~J} / \mathrm{cm}^{2}$ & NI & $60 \mathrm{~s}$ & $\begin{array}{c}2 \\
(0 \text { and } \\
7 \text { th day })\end{array}$ \\
\hline $\begin{array}{c}\text { Hill et al., } \\
2019 \\
\text { (Germany) } \\
{[80]}\end{array}$ & $\begin{array}{l}\text { Indocyanine } \\
\text { green } \\
(0.1 \mathrm{mg} / \mathrm{mL})\end{array}$ & $60 \mathrm{~s}$ & $808 \mathrm{~nm}$ & NI & NI & $100 \mathrm{~mW}$ & NI & NI & NI & $\begin{array}{l}\text { Tip diameter: } \\
\quad 300 \mu \mathrm{m}\end{array}$ & $2829 \mathrm{~J} / \mathrm{cm}^{2}$ & NI & NI & 1 \\
\hline $\begin{array}{l}\text { Ahad et al., } \\
2016 \\
\text { (India) [81] }\end{array}$ & $\begin{array}{l}\text { Phenothiazine } \\
\text { chloride }\end{array}$ & $3 \mathrm{~min}$ & $660 \mathrm{~nm}$ & Contact mode & NI & NI & NI & NI & NA & $\begin{array}{l}\text { Tip diameter: } \\
0.6 \mu \mathrm{m}\end{array}$ & NI & $\begin{array}{c}100 \\
\mathrm{~mW} / \mathrm{cm}^{2}\end{array}$ & $\begin{array}{c}10 \mathrm{~s} / \text { site }(6 \text { sites } \\
1 \mathrm{~min} / \text { tooth })\end{array}$ & 1 \\
\hline $\begin{array}{c}\text { Balata et al., } \\
2013 \\
\text { (Brazil) [82] }\end{array}$ & $\begin{array}{c}\text { Methylene } \\
\text { blue } \\
0.005 \%\end{array}$ & $2 \min$ & $660 \mathrm{~nm}$ & $\begin{array}{l}90^{\circ} \text { angle with the } \\
\text { gingival surface } \\
\text { and with no contact } \\
\text { with the tissues }\end{array}$ & $9 \mathrm{~J}$ & $100 \mathrm{~mW}$ & NI & NI & NI & $\begin{array}{l}\text { Tip diameter: } \\
600 \mu \mathrm{m} \text { tip }\end{array}$ & $320 \mathrm{~J} / \mathrm{cm}^{2}$ & NI & $90 \mathrm{~s} /$ site & 1 \\
\hline $\begin{array}{l}\text { Bechara } \\
\text { et al., 2018 } \\
\text { (Brazil) [83] }\end{array}$ & $\begin{array}{c}\text { Methylene } \\
\text { Blue } \\
(10 \mathrm{mg} / \mathrm{mL})\end{array}$ & $1 \mathrm{~min}$ & $660 \mathrm{~nm}$ & NI & NI & $60 \mathrm{~mW}$ & NI & NI & NI & NI & $129 \mathrm{~J} / \mathrm{cm}^{2}$ & NI & $\begin{array}{l}60 \mathrm{~s} / \text { tooth } \\
(2 \mathrm{sites} / \text { tooth })\end{array}$ & 1 \\
\hline $\begin{array}{c}\text { Bundidpun } \\
\text { et al., } 2017 \\
\text { (Thailand) } \\
{[84]}\end{array}$ & $\begin{array}{l}\text { Phenothiazine } \\
\text { chloride }\end{array}$ & $1 \mathrm{~min}$ & $660 \mathrm{~nm}$ & Contact mode & NI & $100 \mathrm{~mW}$ & NI & NI & NA & NI & $\mathrm{NI}$ & NI & $\begin{array}{c}10 \mathrm{~s} / \mathrm{site} \\
(6 \mathrm{sites}) \\
1 \mathrm{~min} / \text { tooth }\end{array}$ & 1 \\
\hline $\begin{array}{l}\text { Chitsazi } \\
\text { et al., 2014 } \\
\text { (Iran) [85] }\end{array}$ & $\begin{array}{l}\text { Toluidine } \\
\text { Blue }\end{array}$ & $1 \mathrm{~min}$ & $670-690 \mathrm{~nm}$ & Contact mode & NI & $75 \mathrm{~mW}$ & NI & NI & NA & NI & $\mathrm{NI}$ & NI & $120 \mathrm{~s} / \mathrm{site}$ & 1 \\
\hline
\end{tabular}


Table 2. Cont.

\begin{tabular}{|c|c|c|c|c|c|c|c|c|c|c|c|c|c|c|}
\hline $\begin{array}{l}\text { Study, Year, } \\
\text { Origin and } \\
\text { Citation }\end{array}$ & $\begin{array}{l}\text { Photosensi- } \\
\text { tizer (PS) } \\
\text { Used and } \\
\text { Its Concen- } \\
\text { tration }\end{array}$ & $\begin{array}{c}\text { Pre- } \\
\text { Irradiation } \\
\text { Exposure } \\
\text { Time to PS } \\
\text { (min) }\end{array}$ & $\begin{array}{l}\text { Laser } \\
\text { Wave- } \\
\text { length } \\
\text { Utilized }\end{array}$ & $\begin{array}{l}\text { Emission Mode } \\
\text { Contact/No } \\
\text { Contact } \\
\text { Tip Initiation }\end{array}$ & $\begin{array}{c}\text { Energy } \\
\text { (J) }\end{array}$ & $\begin{array}{l}\text { Power } \\
\text { Output } \\
\text { (W) }\end{array}$ & $\begin{array}{c}\text { Pulse } \\
\text { Length } \\
\text { (Duration), } \\
\text { Pulse } \\
\text { Interval }\end{array}$ & $\begin{array}{l}\text { Use of } \\
\text { Power } \\
\text { Meter }\end{array}$ & $\begin{array}{l}\text { Distance } \\
\text { from } \\
\text { Target }\end{array}$ & $\begin{array}{c}\text { Spot } \\
\text { Size/Fibre-Tip } \\
\text { Diameter/Spot } \\
\text { Diameter }\end{array}$ & $\begin{array}{c}\text { Energy } \\
\text { Density } \\
\text { [Fluence] } \\
\left(\mathrm{J} / \mathrm{cm}^{2}\right)\end{array}$ & $\begin{array}{c}\text { Power } \\
\text { Density } \\
\text { [Irradiance] } \\
\left(\mathrm{W} / \mathrm{cm}^{2}\right)\end{array}$ & $\begin{array}{c}\text { Exposure Time } \\
\text { to Laser } \\
\text { Irradiation } \\
\text { [Minute (min)/ } \\
\text { Second (s)] }\end{array}$ & $\begin{array}{l}\text { No. of } \\
\text { aPDT Ap- } \\
\text { plications }\end{array}$ \\
\hline $\begin{array}{l}\text { Chitsazi } \\
\text { et al., 2014 } \\
\text { (Iran) [86] }\end{array}$ & $\begin{array}{c}\text { Tolonium } \\
\text { chloride } \\
\text { (Toluidine } \\
\text { Blue O) }\end{array}$ & $60 \mathrm{~s}$ & $638 \mathrm{~nm}$ & Contact mode & NI & NI & NI & NI & NA & NI & $8-10 \mathrm{~J} / \mathrm{cm}^{2}$ & NI & $120 \mathrm{~s}$ & 1 \\
\hline $\begin{array}{c}\text { Garcia et al., } \\
2011 \\
\text { (Brazil) [87] }\end{array}$ & $\begin{array}{c}\text { Methylene } \\
\text { blue } \\
(0.005 \%)\end{array}$ & $5 \mathrm{~min}$ & $660 \mathrm{~nm}$ & NI & NI & $40 \mathrm{~mW}$ & NI & NI & NI & NI & $120 \mathrm{~J} / \mathrm{cm}^{2}$ & NI & $120 \mathrm{~s} / \mathrm{site}$ & 1 \\
\hline $\begin{array}{c}\text { Joseph et al., } \\
2014 \\
\text { (India) [88] }\end{array}$ & $\begin{array}{l}\text { Methylene } \\
\text { blue } \\
(10 \mathrm{mg} / \mathrm{mL})\end{array}$ & $3 \mathrm{~min}$ & $655 \mathrm{~nm}$ & $\begin{array}{l}\text { CW, contact mode, } \\
\text { tip was inserted } \\
\text { into the gingival } \\
\text { sulcus }\end{array}$ & NI & NI & NI & NI & NA & $\begin{array}{c}\text { Tip diameter: } \\
200 \mu \mathrm{m} \\
\text { Probe tip diameter: } \\
0.5 \mathrm{~mm}\end{array}$ & $\mathrm{NI}$ & $\begin{array}{c}60 \mathrm{~mW} / \\
\mathrm{cm}^{2}\end{array}$ & $\begin{array}{c}60 \mathrm{~s} / \mathrm{site} \\
(4 \mathrm{sites} / \text { tooth })\end{array}$ & 1 \\
\hline $\begin{array}{l}\text { Malgikar } \\
\text { et al., 2015 } \\
\text { (India) [89] }\end{array}$ & $\begin{array}{c}\text { Methylene } \\
\text { blue } \\
1 \%\end{array}$ & $3 \mathrm{~min}$ & $980 \mathrm{~nm}$ & $\begin{array}{l}\text { Contact mode, tip } \\
\text { was initiated }\end{array}$ & NI & $\begin{array}{l}\text { Peak } \\
\text { Power: } \\
5 \mathrm{~W} \\
\text { Average } \\
\text { power } \\
1 \mathrm{~W}\end{array}$ & $\begin{array}{c}\text { Pulse } \\
\text { length: } \\
200 \mu s, \\
\text { Pulse } \\
\text { interval: } \\
200 \\
\end{array}$ & NI & NA & $\begin{array}{l}\text { Tip diameter: } \\
400 \mu \mathrm{m}\end{array}$ & NI & NI & $30-45 \mathrm{~s} /$ site & 1 \\
\hline $\begin{array}{l}\text { Monzavi } \\
\text { et al., 2016 } \\
\text { (Iran) [90] }\end{array}$ & $\begin{array}{l}\text { Indocyanine } \\
\text { green } \\
(1 \mathrm{mg} / \mathrm{mL})\end{array}$ & NI & $810 \mathrm{~nm}$ & $\mathrm{CW}$, contact mode & $\begin{array}{l}\text { PBM } \\
\text { tip: } 6 \mathrm{~J} \\
\text { Bulb } \\
\text { tip: } 4 \mathrm{~J}\end{array}$ & $200 \mathrm{~mW}$ & NI & NI & NA & $\begin{array}{l}\text { Use of two types of } \\
\text { tips: PBM tip was } \\
\text { placed on papilla } \\
\text { and then the bulb } \\
\text { tip was inserted } \\
\text { inside the pocket } \\
\text { from each buccal or } \\
\text { lingual/palatal } \\
\text { side, moving from } \\
\text { the bottom of the } \\
\text { pocket to the } \\
\text { coronal aspect. }\end{array}$ & NI & NI & $\begin{array}{l}\text { PBM tip: } 30 \mathrm{~s} \\
\text { Bulb tip: } 10 \mathrm{~s}\end{array}$ & $\begin{array}{c}4 \\
(0,7 \text { th, } 17 \text { th } \\
\text { and } \\
\text { 27th days })\end{array}$ \\
\hline $\begin{array}{c}\text { Raj et al., } \\
2016 \\
\text { (India) [91] }\end{array}$ & $\begin{array}{l}\text { Toluidine } \\
\text { blue }\end{array}$ & $1 \mathrm{~min}$ & $635 \mathrm{~nm}$ & Contact mode & NI & $500 \mathrm{~W}$ & NI & NI & NA & NI & NI & NI & $60 \mathrm{~s}$ & 1 \\
\hline $\begin{array}{c}\text { Sena et al., } \\
2019 \\
\text { (Brazil) [92] }\end{array}$ & $\begin{array}{l}\text { Chloro- } \\
\text { aluminum } \\
\text { pthalocya- } \\
\text { nine } \\
\text { (AlClFc) } \\
5 \mu \mathrm{M}\end{array}$ & $5 \mathrm{~min}$ & $660 \mathrm{~nm}$ & $\begin{array}{l}\mathrm{CW} \text {, laser optical } \\
\text { fiber tip was } \\
\text { positioned parallel } \\
\text { to the tooth axis in } \\
\text { contact with the } \\
\text { gingival margin } \\
\text { (without } \\
\text { penetrating the } \\
\text { pocket) }\end{array}$ & $1.5 \mathrm{~J}$ & $100 \mathrm{~mW}$ & NI & NI & NA & Spot size: $0.028 \mathrm{~cm}^{2}$ & $54 \mathrm{~J} / \mathrm{cm}^{2}$ & $4 \mathrm{~W} / \mathrm{cm}^{2}$ & $15 \mathrm{~s}$ & 1 \\
\hline
\end{tabular}


Table 2. Cont.

\begin{tabular}{|c|c|c|c|c|c|c|c|c|c|c|c|c|c|c|}
\hline $\begin{array}{l}\text { Study, Year, } \\
\text { Origin and } \\
\text { Citation }\end{array}$ & $\begin{array}{l}\text { Photosensi- } \\
\text { tizer (PS) } \\
\text { Used and } \\
\text { Its Concen- } \\
\text { tration }\end{array}$ & $\begin{array}{c}\text { Pre- } \\
\text { Irradiation } \\
\text { Exposure } \\
\text { Time to PS } \\
\text { (min) }\end{array}$ & $\begin{array}{c}\text { Laser } \\
\text { Wavelength } \\
\text { Utilized }\end{array}$ & $\begin{array}{l}\text { Emission Mode } \\
\text { Contact/No } \\
\text { Contact } \\
\text { Tip Initiation }\end{array}$ & $\begin{array}{c}\text { Energy } \\
\text { (J) }\end{array}$ & $\begin{array}{l}\text { Power } \\
\text { Output } \\
\text { (W) }\end{array}$ & $\begin{array}{c}\text { Pulse } \\
\text { Length } \\
\text { (Duration), } \\
\text { Pulse } \\
\text { Interval }\end{array}$ & $\begin{array}{l}\text { Use of } \\
\text { Power } \\
\text { Meter }\end{array}$ & $\begin{array}{c}\text { Distance } \\
\text { from Target }\end{array}$ & $\begin{array}{c}\text { Spot } \\
\text { Size/Fibre-Tip } \\
\text { Diame- } \\
\text { ter/Spot } \\
\text { Diameter } \\
\end{array}$ & $\begin{array}{c}\text { Energy } \\
\text { Density } \\
\text { [Fluence] } \\
\left(\mathrm{J} / \mathrm{cm}^{2}\right)\end{array}$ & $\begin{array}{c}\text { Power } \\
\text { Density } \\
\text { [Irradiance] } \\
\left(\mathrm{W} / \mathrm{cm}^{2}\right)\end{array}$ & $\begin{array}{c}\text { Exposure Time } \\
\text { to Laser } \\
\text { Irradiation } \\
\text { [Minute (min)/ } \\
\text { Second (s)] }\end{array}$ & $\begin{array}{l}\text { No. of } \\
\text { aPDT Ap } \\
\text { plications }\end{array}$ \\
\hline $\begin{array}{c}\text { Sigusch } \\
\text { et al., 2010 } \\
\text { (Germany) } \\
{[94]} \\
\end{array}$ & $\begin{array}{l}\text { Phenothiazine } \\
\text { chloride }\end{array}$ & $1 \mathrm{~min}$ & $660 \mathrm{~nm}$ & Contact mode & NI & $\mathrm{NI}$ & $\mathrm{NI}$ & $\mathrm{NI}$ & NA & $\begin{array}{l}\text { Tip diameter: } \\
600 \mu \mathrm{m} \text { tip }\end{array}$ & $\mathrm{NI}$ & $60 \mathrm{~mW} / \mathrm{cm}^{2}$ & $\begin{array}{l}10 \mathrm{~s} / \text { site }(6 \text { sites } \\
=1 \mathrm{~min} / \text { tooth })\end{array}$ & 1 \\
\hline $\begin{array}{l}\text { Theodoro } \\
\text { et al., 2012 } \\
\text { (Brazil) [95] }\end{array}$ & $\begin{array}{c}\text { Toluidine } \\
\text { blue O } \\
100 \mu \mathrm{g} / \mathrm{mL}\end{array}$ & $1 \mathrm{~min}$ & $660 \mathrm{~nm}$ & $\begin{array}{l}\text { The laser optical } \\
\text { fiber tip was } \\
\text { positioned parallel } \\
\text { to and in contact } \\
\text { with the } \\
\text { selected site }\end{array}$ & $4.5 \mathrm{~J}$ & $30 \mathrm{~mW}$ & NI & $\mathrm{NI}$ & NA & $\begin{array}{l}\text { Spot size: } \\
0.07 \mathrm{~cm}^{2}\end{array}$ & $64.28 \mathrm{~J} / \mathrm{cm}^{2}$ & $0.4 \mathrm{~W} / \mathrm{cm}^{2}$ & $150 \mathrm{~s}$ & 1 \\
\hline $\begin{array}{l}\text { Theodoro } \\
\text { et al., 2017 } \\
\text { (Brazil) [96] }\end{array}$ & $\begin{array}{c}\text { Methylene } \\
\text { blue } \\
(10 \mathrm{mg} / \mathrm{mL})\end{array}$ & $1 \mathrm{~min}$ & $660 \mathrm{~nm}$ & Contact mode & $4.8 \mathrm{~J}$ & $100 \mathrm{~mW}$ & $\mathrm{NI}$ & NI & NA & $\begin{array}{l}\text { Spot size } \\
0.03 \mathrm{~cm}^{2}\end{array}$ & $160 \mathrm{~J} / \mathrm{cm}^{2}$ & NI & $48 \mathrm{~s}$ & $\begin{array}{c}3 \\
(0,48 \mathrm{~h}, \\
96 \mathrm{~h})\end{array}$ \\
\hline
\end{tabular}




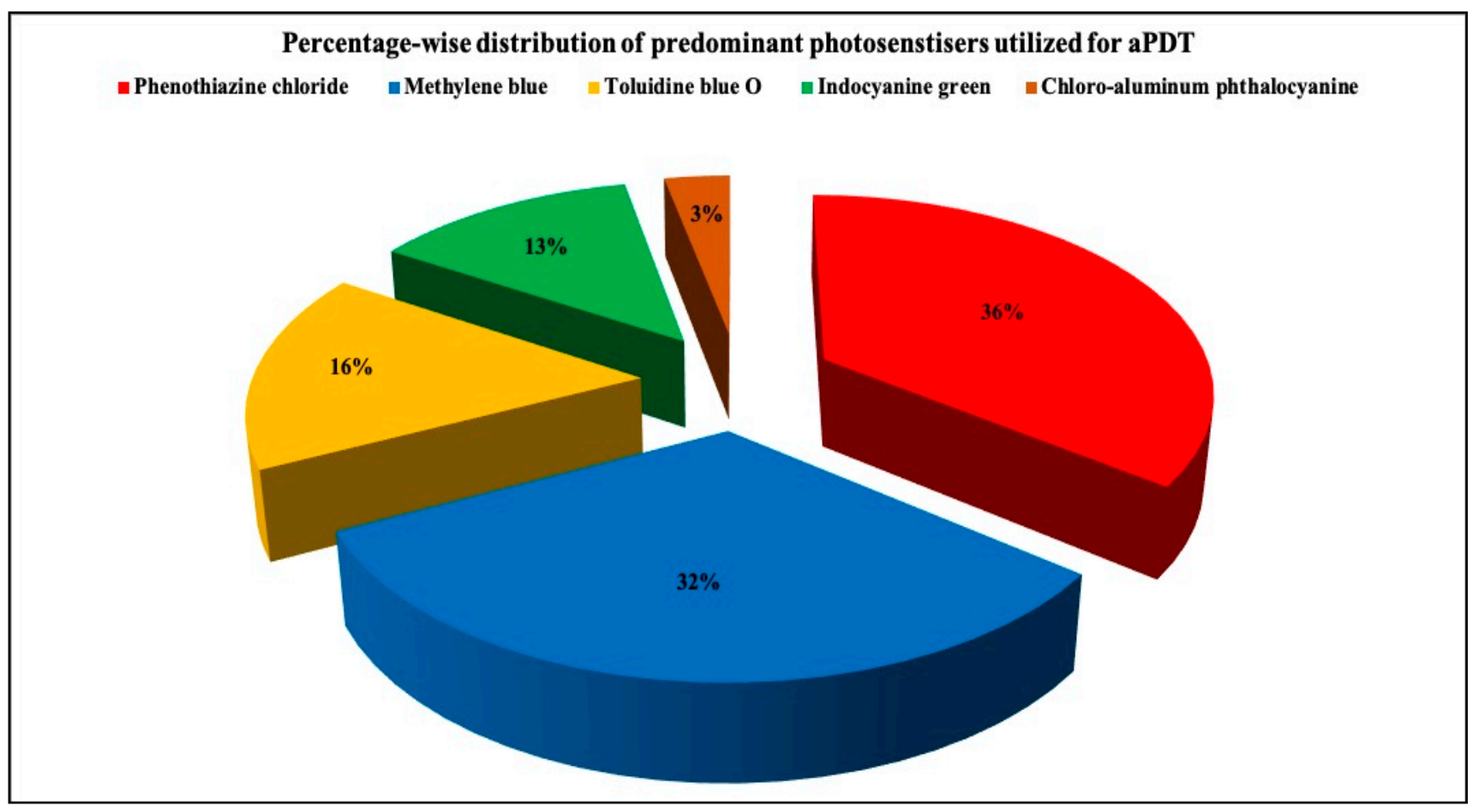

Figure 3. 3D pie diagram illustrating the percentage-wise distribution of predominant photosensitizers utilized for aPDT in the included studies.

3.2.6. Utilization of aPDT as a Mono-Therapeutic or an Adjunctive Therapeutic Agent

While 28 out of the 31 eligible studies utilized SRP+aPDT, aPDT monotherapy was performed in three studies $[2,3,5]$ (Table 1 ).

\subsubsection{Comparison in between $\mathrm{SRP}+\mathrm{aPDT}$ versus $\mathrm{SRP}+\mathrm{AB}$}

Six out of the 31 eligible studies compared efficacy of SRP+aPDT versus SRP+ AB $[72-74,79,83,96]$ (Table 1$)$.

\subsubsection{Number of aPDT Sessions}

While a single session of aPDT was applied in 22 out of the 31 included studies $[2,3,5,70,76-78,80-89,91-95]$, multiple aPDT sessions were performed in nine studies $[17,71-75,79,90,96]$. None of the eligible studies compared single versus multiple sessions of aPDT (Table 2).

\subsubsection{Follow-Up Assessment}

A follow-up assessment at three months from the baseline visit was performed in 18 out of the 31 eligible studies $[2,3,5,17,70,71,74,76,81,85-87,90-94,96]$, whereas 12 studies conducted a longer follow-up assessment at six months $[72,73,75,77,78,80,82-84,88,89,95]$. Only one study performed a follow-up assessment at one month from the baseline visit [79]. A long-term follow-up of a minimum one year from baseline visit lacked in all eligible studies.

\subsection{Qualitative Assessment}

Qualitative assessment was performed using the RoB 2 tool, designed for in vivo human RCTs, as depicted in Figures 4 and 5. The most recent version of this tool was utilized to perform a qualitative assessment for both randomized PG and SM human RCTs $[29,30]$. Figure 4 represents a risk of bias assessment summary of all eligible studies. Figure 5 is a graphical representation of percentage RoB score for each risk domain, which has been evaluated, using the abovementioned tool. Furthermore, $53.1 \%$ of included trials 
were at a high risk of inadequate randomization, whereas $40.6 \%$ and $6.3 \%$ of included trials were at a low risk or had some concerns, respectively. In addition, $50 \%$ of included studies were at a high risk of deviations from intended interventions, whereas $43.7 \%$ and $6.3 \%$ of them were at a low risk or had some concerns, respectively. All included papers reported substantial evidence $(100 \%)$ for reporting missing outcome data and, hence, were at a low risk. Although a majority of studies were free of bias arising from reporting outcome measurement $(71.9 \%), 28.1 \%$ were at a high risk. In terms of selective reporting of the results, inferences are as follows: $59.4 \%$ studies were at a high risk, $37.5 \%$ studies were at a low risk, and $3.1 \%$ studies had some concerns. Overall, $60 \%$ studies reported a high risk of bias, while $35 \%$ studies had a low risk of bias, and the final $5 \%$ studies had some concerns. It should be noted that information provided in these figures represents the consensual answers verified using the 'Discrepancy check' feature of RoB 2 tool, across two independent reviewers (S.D. and R.H.) (inter-reviewer agreement, $k=0.94$ ), and, in case of any disagreements, a third author (S.B.) was consulted.

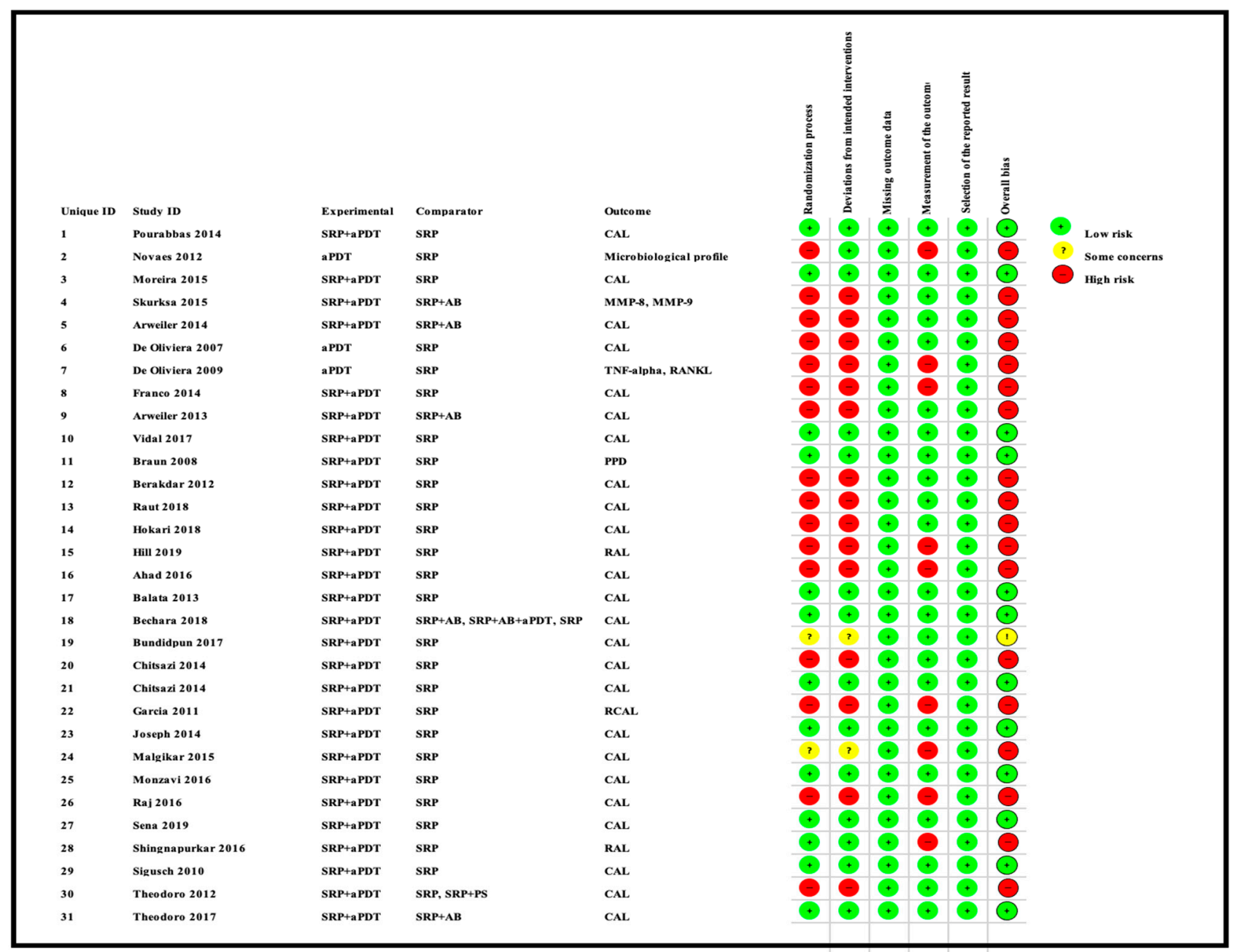

Figure 4. Risk of Bias assessment summary of the included studies based on the consensual answers across two individual assessors (S.D. and R.H.). 


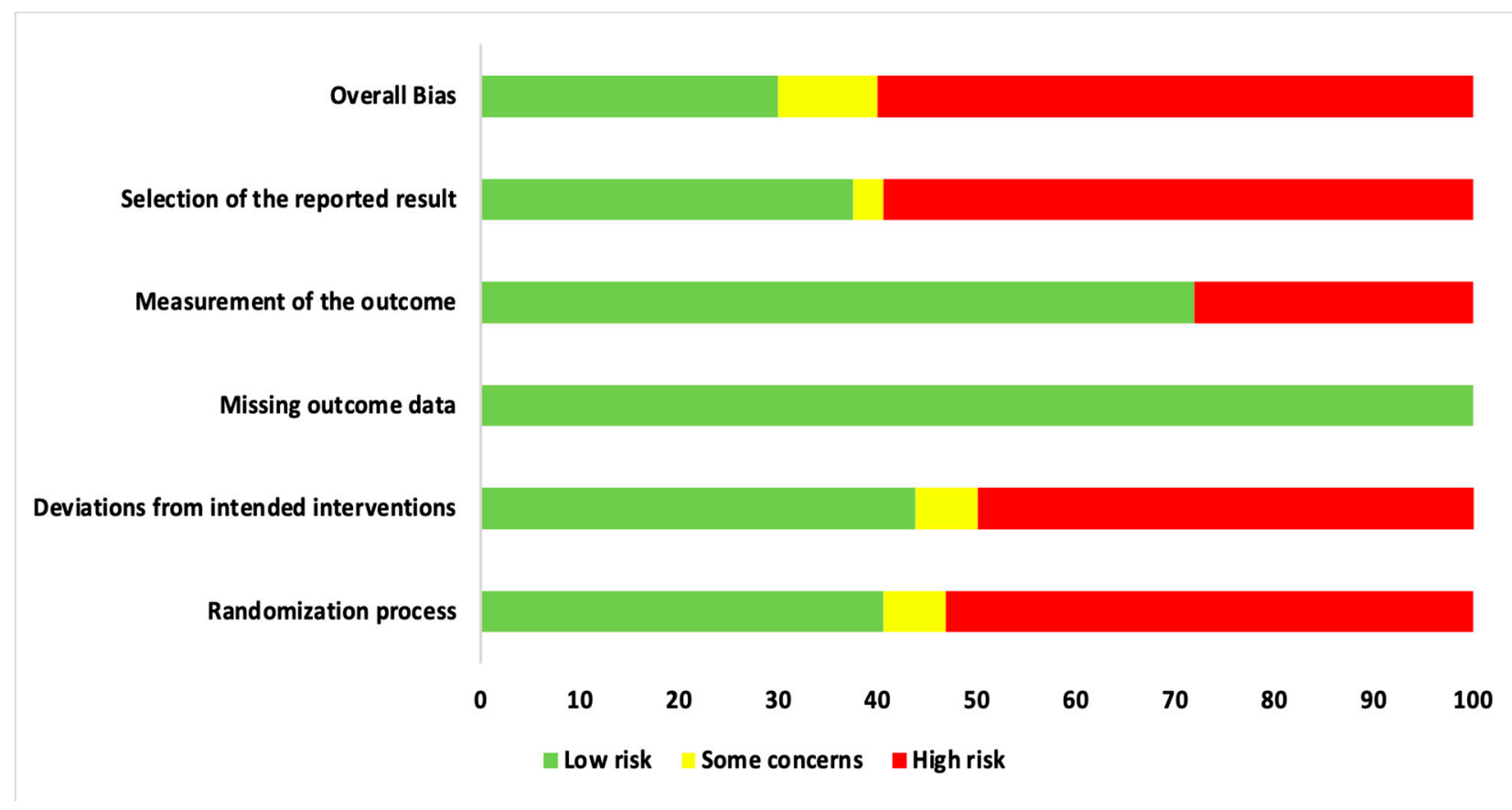

Figure 5. Risk of Bias assessment graph of the included studies expressed as percentages, based on the consensual answers across two individual assessors (S.D. and R.H.).

\subsection{Quantitative Assessment}

\subsubsection{Outcome Variables}

Primary outcomes of 18 out of 31 studies, which have assessed efficacy of SRP+aPDT in the management of periodontitis, contributed to this meta-analysis $[17,71,73-76,80,81,84-$ 86,88-93,96]. Data were pooled separately for SM and PG studies for differences in PPD and CAL respectively at three and six months, respectively. At three months, the mean difference in PPD reduction was not statistically significant for SM studies (SMD 0.166; 95\% CI -0.278 to $0.611 ; P=0.463$ ) and PG studies (SMD $0.076 ; 95 \% \mathrm{CI}-0.420$ to $0.573 ; P=0.763$ ) along with a high heterogeneity for $\mathrm{SM}$ studies $\left(\mathrm{Q}=15.81 ; P=0.0001 ; \mathrm{I}^{2}=91.21 \%\right)$ and moderate heterogeneity $\left(\mathrm{Q}=11.87 ; P=0.018 ; \mathrm{I}^{2}=66.31 \%\right)$ for PG studies (Table 3 ). The mean difference in PPD reduction at six months did not show a statistically significant difference for SM studies (SMD 0.005; 95\% CI -0.126 to 0.136 ; $P=0.935$ ) as well as PG studies (SMD 0.141; $95 \% \mathrm{CI}-1.007$ to $1.288 ; P=0.809$ ) although contrasting findings were noted in terms of level of heterogeneity which was not evident for SM studies $(\mathrm{Q}=0.06$; $\left.P=0.99 ; \mathrm{I}^{2}=0.00 \%\right)$ and high for PG studies $\left(\mathrm{Q}=18.71 ; \mathrm{P}=0.0001 ; \mathrm{I}^{2}=89.31 \%\right)($ Table 4$)$. CAL gain at three months was not statistically significant in SM studies (SMD 0.092;95\% $\mathrm{CI}-0.013$ to $0.198 ; P=0.088)$ with no evident heterogeneity $\left(\mathrm{Q}=8.74 ; P=0.655 ; \mathrm{I}^{2}=0.00 \%\right)$ as well as in PG studies (SMD 0.056; $95 \% \mathrm{CI}-0.408$ to $0.552 ; P=0.745)$ with moderate heterogeneity $\left(\mathrm{Q}=8.95 ; P=0.028 ; \mathrm{I}^{2}=70.31 \%\right)$ (Table 3$)$. At six months, results for SM studies were not statistically significant (SMD $-0.013 ; 95 \% \mathrm{CI}-0.148$ to $0.121 ; P=0.846$ ) with no evident heterogeneity $\left(\mathrm{Q}=0.03 ; P=0.984 ; \mathrm{I}^{2}=0.00 \%\right)$, whereas, for PG studies, the findings were statistically significant (SMD $-0.441 ; 95 \% \mathrm{CI}-0.805$ to $-0.075 ; P=0.018$ ) with no evidence of heterogeneity $\left(\mathrm{Q}=1.70 ; P=0.42 ; \mathrm{I}^{2}=0.00 \%\right)$ but favoring control group (Table 4). 
Table 3. Forest plots illustrating the overall PPD reduction and CAL gain at three months. Refer to Supplementary file 2 for a list of abbreviations.

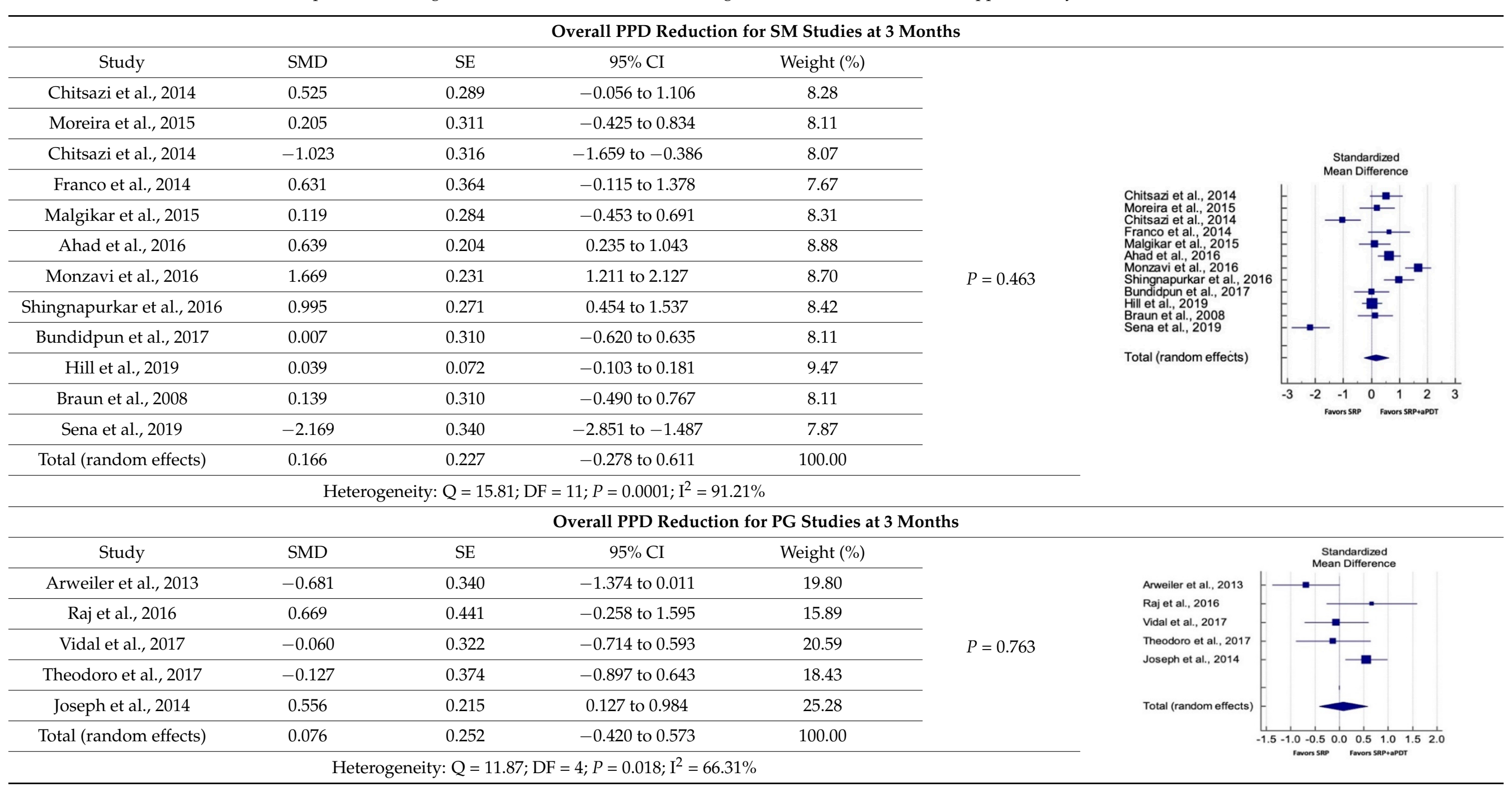


Table 3. Cont.

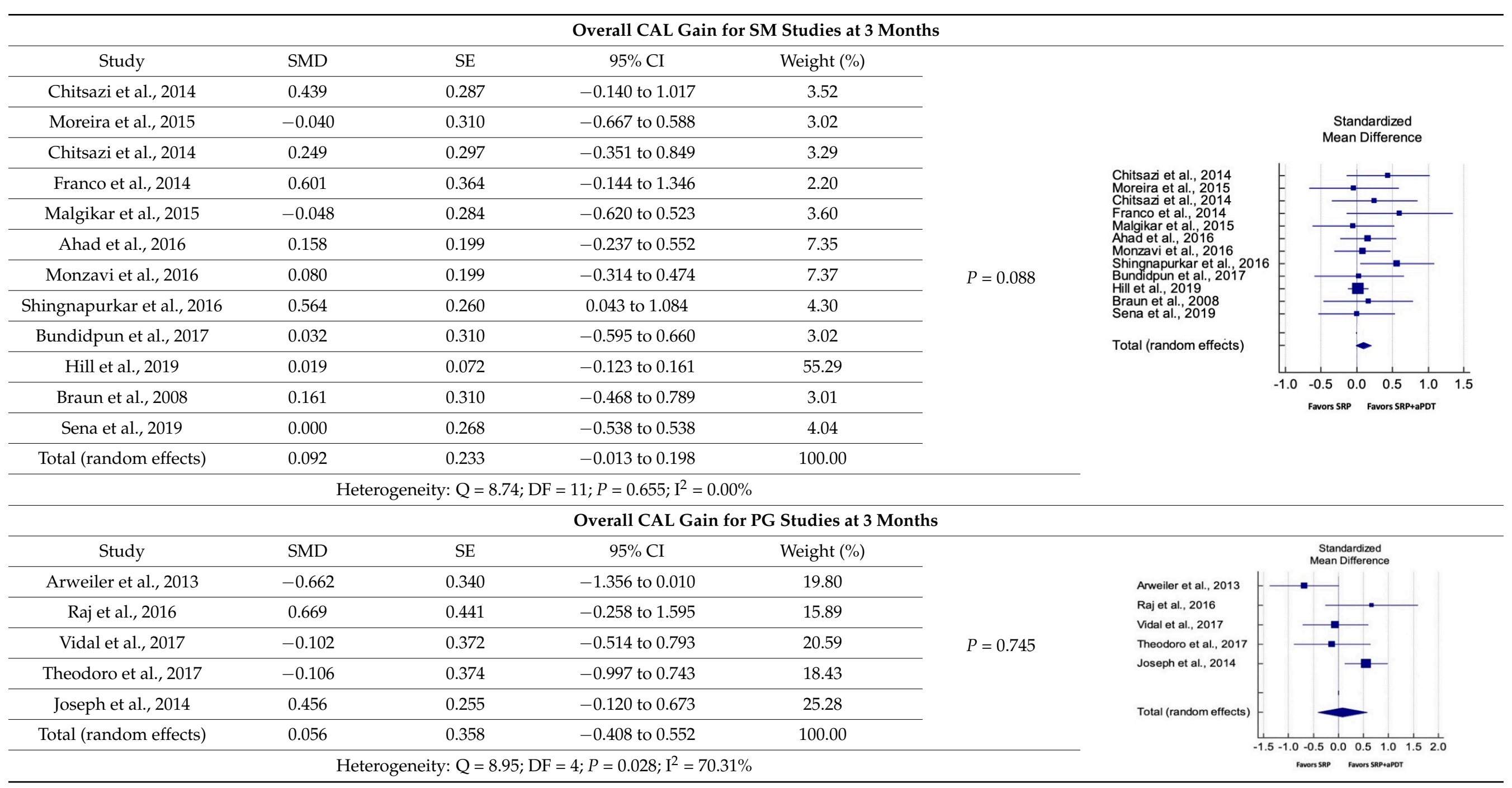


Table 4. Forest plots illustrating the overall PPD reduction and CAL gain at 6 months. Refer to Supplementary file 2 for a list of abbreviations.

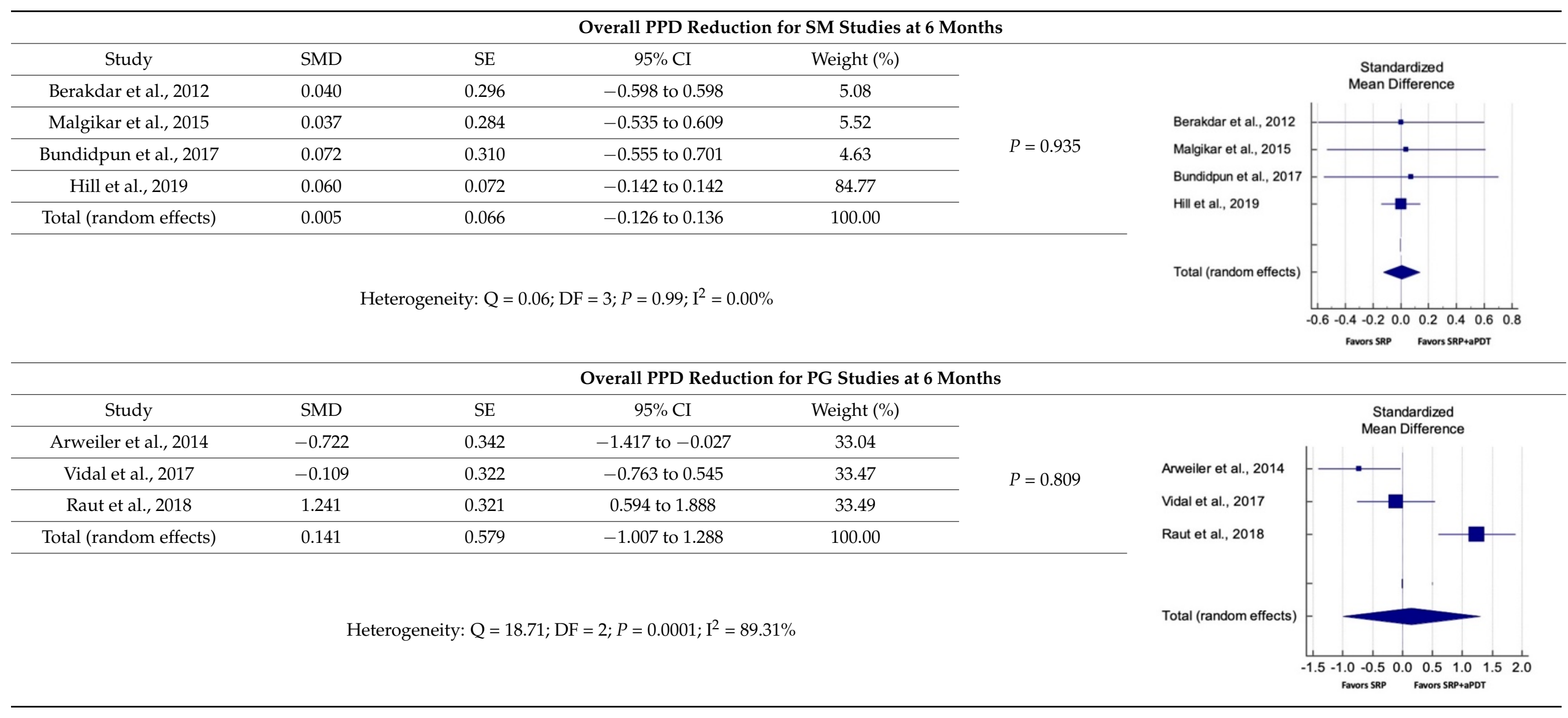


Table 4. Cont.

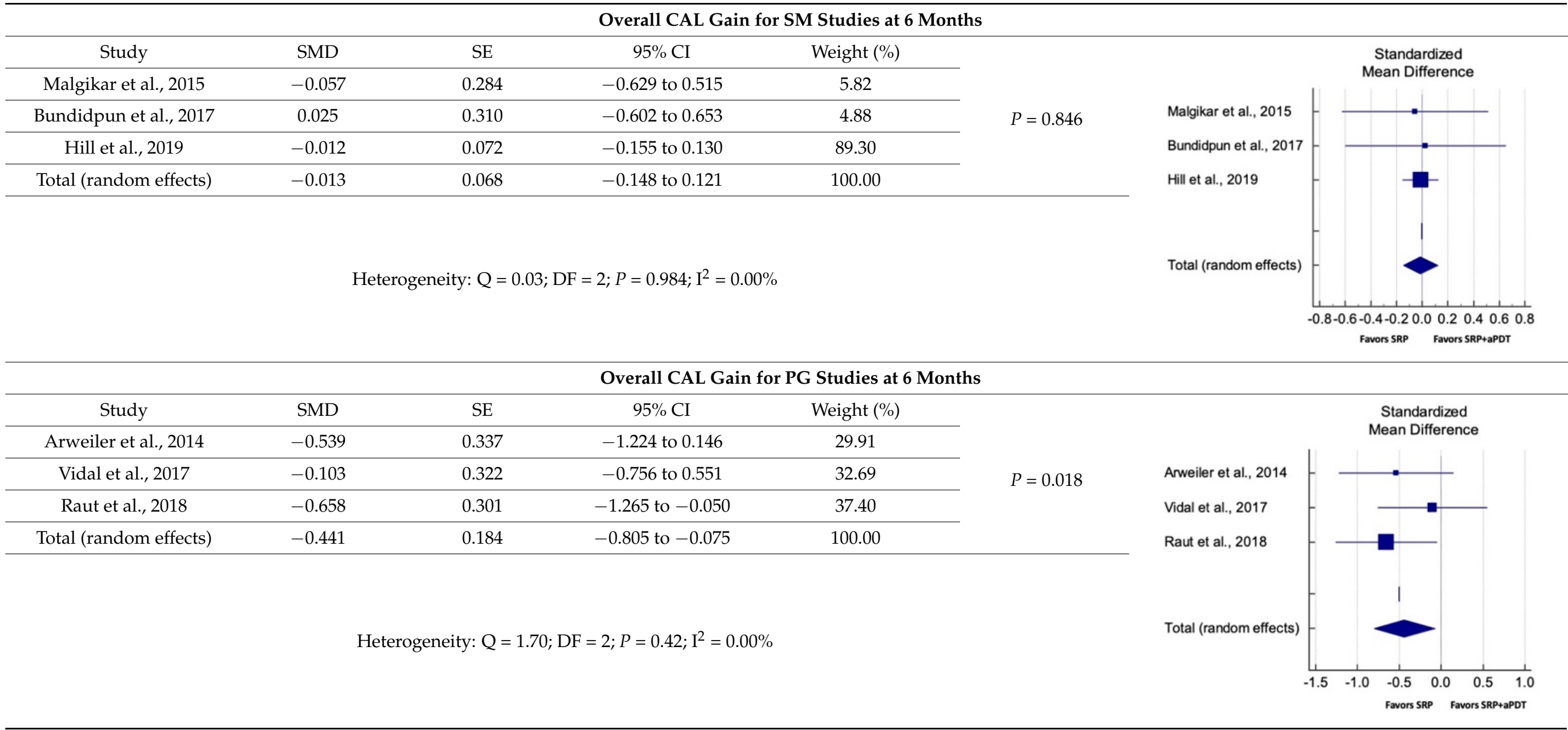


Assessment of secondary outcome variables was conducted in the majority of included studies, which are as follows: Changes in GR, BOP, PI and GI in 28 studies [3,17,70,71,73-96], microbiological analysis in 11 studies [5,71,75,78-80,85,86,91,94,95], and immuno-histological in seven studies $[2,17,70-72,75,79]$. Table 5 provides an overview of clinical parameters which have been assessed in 28 of 31 included studies along with corresponding level of significance, in accordance to data provided in Table 1. Eleven studies performed a microbiological analysis $[5,71,75,78-80,85,86,91,94,95]$, out of which five studies reported that aPDT therapy could significantly reduce periopathogenic burden $[71,75,78,91,94]$ and six studies failed to achieve this outcome $[5,79,80,85,86,95]$. In terms of immune-histological analysis, seven studies $[2,17,70-72,75,79]$ assessed various pro-inflammatory cytokines and growth factors such as; IL-1 $\beta$, IL-10, IF- $\gamma$, TNF- $\alpha$, MMP-8, MMP-9, RANK, RANK-L, OPG and FGF-2 (Table 1). Biomarkers for assessment of bone resorption (RANK, RANK-L, OPG) were assessed in three studies $[2,17,75]$. Two studies $[17,75]$ assessed efficacy of SRP+aPDT in comparison to conventional SRP alone, and showed that SRP+aPDT successfully suppressed the bone resorption process. Levels of IL-1 $\beta$, IL-10, IF- $\gamma$, and TNF- $\alpha$ were assessed in three studies $[70,71,79]$, of which two studies have confirmed immunological benefits of aPDT [70,71], whereas one study [79] failed to show any advantage of aPDT for the same. It should, however, be noted that, while the former two studies [70,71] have compared the efficacy of SRP+aPDT to conventional SRP alone, the latter study [79] has compared $\mathrm{SRP}+\mathrm{aPDT}$ to $\mathrm{SRP}+\mathrm{AB}$ and demonstrated the advantages of AB over aPDT. Additionally, $\mathrm{SRP}+\mathrm{aPDT}$ showed an increased expression of FGF-2, which plays a role in tissue repair as compared to SRP alone, and was assessed in only one study [17]. A meta-analysis on secondary outcomes was not possible due to disparity in scoring methodology, incomplete, or incomparable data.

\subsubsection{Sensitivity Analysis}

A sensitivity analysis was conducted due to the noteworthy heterogeneity arising from outlier studies which were detected upon visual inspection of Forest plots [74,86,90,92,93] (Tables 3 and 4). This analysis was conducted only for the three-month follow-up due to unavailability of data in included studies (Table 6). In terms of PPD reduction, SM studies (SMD 0.282; 95\% CI -0.286 to $0.624 ; P=0.153$ ) as well as PG studies (SMD 0.257; $95 \%$ CI -0.230 to $0.683 ; P=0.361$ ) did not report a statistically significant improvement. No evident heterogeneity $\left(\mathrm{Q}=9.14 ; \mathrm{P}=0.7 ; \mathrm{I}^{2}=0.00 \%\right)$ in SM studies and in PG studies $\left(\mathrm{Q}=8.87 ; P=0.22 ; \mathrm{I}^{2}=0.00 \%\right.$ ) was noted (Table 6$)$. Although improvement in CAL gain was noted after omitting outlier studies, this difference was statistically not significant in both SM (SMD 0.162; 95\% CI - 0.326 to $0.406 ; P=0.166$ ) and PG studies (SMD $0.227 ; 95 \% \mathrm{CI}$ -0.420 to $0.673 ; P=0.234)$ with no evident heterogeneity $\left(Q=8.40 ; P=0.625 ; I^{2}=0.00 \%\right)$ in SM studies as well as in PG studies $\left(Q=9.7 ; P=0.22 ; \mathrm{I}^{2}=0.00 \%\right)$ (Table 6). 


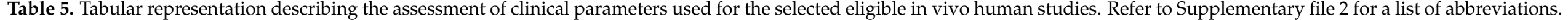

\begin{tabular}{|c|c|c|c|c|c|c|c|c|c|c|c|c|}
\hline \multirow[b]{2}{*}{$\begin{array}{l}\text { Study, Year, Origin } \\
\text { and Citation }\end{array}$} & \multicolumn{2}{|c|}{ PPD } & \multicolumn{2}{|c|}{ CAL } & \multicolumn{2}{|c|}{ BOP/SBI } & \multicolumn{2}{|c|}{ PI } & \multicolumn{2}{|c|}{ GI } & \multicolumn{2}{|c|}{ GR } \\
\hline & $\begin{array}{l}\text { Statistically } \\
\text { Significant } \\
\text { Y/N/NI/NS }\end{array}$ & $\begin{array}{c}\text { Not } \\
\text { Statistically } \\
\text { Significant } \\
\text { Y/N/NI/NS }\end{array}$ & $\begin{array}{l}\text { Statistically } \\
\text { Significant } \\
\text { Y/N/NI/NS }\end{array}$ & \begin{tabular}{l}
\multicolumn{1}{c}{ Not } \\
Statistically \\
Significant \\
Y/N/NI/NS
\end{tabular} & $\begin{array}{l}\text { Statistically } \\
\text { Significant } \\
\text { Y/N/NI/NS }\end{array}$ & $\begin{array}{c}\text { Not } \\
\text { Statistically } \\
\text { Significant } \\
\text { Y/N/NI/NS }\end{array}$ & $\begin{array}{l}\text { Statistically } \\
\text { Significant } \\
\text { Y/N/NI/NS }\end{array}$ & $\begin{array}{c}\text { Not } \\
\text { Statistically } \\
\text { Significant } \\
\text { Y/N/NI/NS }\end{array}$ & $\begin{array}{l}\text { Statistically } \\
\text { Significant } \\
\text { Y/N/NI/NS }\end{array}$ & $\begin{array}{c}\text { Not } \\
\text { Statistically } \\
\text { Significant } \\
\text { Y/N/NI/NS }\end{array}$ & $\begin{array}{l}\text { Statistically } \\
\text { Significant } \\
\text { Y/N/NI/NS }\end{array}$ & $\begin{array}{c}\text { Not } \\
\text { Statistically } \\
\text { Significant } \\
\text { Y/N/NI/NS }\end{array}$ \\
\hline $\begin{array}{l}\text { Franco et al., } 2014 \\
\text { (Brazil) [17] }\end{array}$ & $\mathrm{N}$ & $Y$ & $\mathrm{~N}$ & $Y$ & $\mathrm{Y}$ & $\mathrm{N}$ & $\mathrm{N}$ & $Y$ & NS & NS & NS & NS \\
\hline $\begin{array}{l}\text { Pourabbas et al., } 2014 \\
\text { (Iran) [70] }\end{array}$ & $\mathrm{N}$ & $Y$ & $\mathrm{~N}$ & $Y$ & $\mathrm{~N}$ & $\mathrm{Y}$ & NS & NS & NS & NS & $\mathrm{N}$ & Y \\
\hline $\begin{array}{l}\text { Moreira et al., } 2015 \\
\text { (Brazil) [71] }\end{array}$ & $\mathrm{Y}$ & $\mathrm{N}$ & Y & $\mathrm{N}$ & $\mathrm{Y}$ & $\mathrm{N}$ & $\mathrm{Y}$ & $\mathrm{N}$ & NS & NS & $Y$ & $\mathrm{~N}$ \\
\hline $\begin{array}{c}\text { Arweiler et al., } 2014 \\
\text { (Poland) [73] }\end{array}$ & $\mathrm{N}$ & $Y$ & $\mathrm{~N}$ & $Y$ & $\mathrm{~N}$ & $\mathrm{Y}$ & $\mathrm{N}$ & $Y$ & NS & NS & $\mathrm{N}$ & Y \\
\hline $\begin{array}{l}\text { Arweiler et al., } 2013 \\
\text { (Poland) [74] }\end{array}$ & $\mathrm{N}$ & $Y$ & $\mathrm{~N}$ & $Y$ & $\mathrm{~N}$ & $\mathrm{Y}$ & $\mathrm{N}$ & Y & NS & NS & $\mathrm{N}$ & $Y$ \\
\hline $\begin{array}{l}\text { Vidal et al., } 2017 \\
\text { (Spain) [75] }\end{array}$ & $\mathrm{N}$ & $Y$ & $\mathrm{~N}$ & $\mathrm{Y}$ & $\mathrm{N}$ & $\mathrm{Y}$ & $\mathrm{N}$ & Y & NS & NS & $\mathrm{N}$ & $Y$ \\
\hline $\begin{array}{c}\text { Braun et al., 2008 } \\
\text { (Germany) [76] }\end{array}$ & $\mathrm{Y}$ & $\mathrm{N}$ & $Y$ & $\mathrm{~N}$ & $\mathrm{Y}$ & $\mathrm{N}$ & NS & NS & NS & NS & $\mathrm{N}$ & Y \\
\hline $\begin{array}{c}\text { Raut et al., } 2018 \\
\text { (India) [78] }\end{array}$ & $\mathrm{Y}$ & $\mathrm{N}$ & Y & $\mathrm{N}$ & $\mathrm{Y}$ & $\mathrm{N}$ & $\mathrm{N}$ & $Y$ & NS & NS & NS & NS \\
\hline $\begin{array}{c}\text { Hokari et al., } 2018 \\
\text { (Japan) [79] }\end{array}$ & $\mathrm{N}$ & $Y$ & $\mathrm{~N}$ & $Y$ & $\mathrm{~N}$ & $\mathrm{Y}$ & $\mathrm{N}$ & $Y$ & $\mathrm{~N}$ & Y & NS & NS \\
\hline $\begin{array}{l}\text { Hill et al., 2019 } \\
\text { (Germany) [80] }\end{array}$ & $\mathrm{N}$ & $Y$ & $\mathrm{~N}$ & $Y$ & $\mathrm{~N}$ & $\mathrm{Y}$ & NS & NS & NS & NS & $\mathrm{N}$ & Y \\
\hline $\begin{array}{c}\text { Ahad et al., } 2016 \\
\text { (India) [81] }\end{array}$ & $\mathrm{N}$ & $Y$ & $\mathrm{~N}$ & $\mathrm{Y}$ & $\mathrm{Y}$ & $\mathrm{N}$ & $\mathrm{N}$ & Y & NS & NS & NS & NS \\
\hline $\begin{array}{l}\text { Balata et al., } 2013 \\
\text { (Brazil) [82] }\end{array}$ & $\mathrm{N}$ & Y & $\mathrm{N}$ & $Y$ & $\mathrm{~N}$ & $\mathrm{Y}$ & $\mathrm{N}$ & Y & $\mathrm{N}$ & $Y$ & $\mathrm{~N}$ & Y \\
\hline $\begin{array}{c}\text { Bechara et al., } 2018 \\
\text { (Brazil) [83] }\end{array}$ & $\mathrm{Y}$ & $\mathrm{N}$ & $Y$ & $\mathrm{~N}$ & $\mathrm{Y}$ & $\mathrm{N}$ & NS & NS & NS & NS & $\mathrm{Y}$ & $\mathrm{N}$ \\
\hline $\begin{array}{l}\text { Bundidpun et al., } \\
2017 \text { (Thailand) [84] }\end{array}$ & $\mathrm{N}$ & $Y$ & $\mathrm{~N}$ & $\mathrm{Y}$ & $\mathrm{Y}$ & $\mathrm{N}$ & $\mathrm{N}$ & Y & Y & $\mathrm{N}$ & NS & NS \\
\hline $\begin{array}{l}\text { Chitsazi et al., } 2014 \\
\text { (Iran) [85] }\end{array}$ & $\mathrm{N}$ & $Y$ & $\mathrm{~N}$ & $\mathrm{Y}$ & $\mathrm{N}$ & $\mathrm{Y}$ & $\mathrm{N}$ & Y & $\mathrm{N}$ & Y & $\mathrm{N}$ & Y \\
\hline
\end{tabular}


Table 5. Cont.

\begin{tabular}{|c|c|c|c|c|c|c|c|c|c|c|c|c|}
\hline \multirow[b]{2}{*}{$\begin{array}{l}\text { Study, Year, Origin } \\
\text { and Citation }\end{array}$} & \multicolumn{2}{|c|}{ PPD } & \multicolumn{2}{|c|}{ CAL } & \multicolumn{2}{|c|}{ BOP/SBI } & \multicolumn{2}{|c|}{ PI } & \multicolumn{2}{|c|}{ GI } & \multicolumn{2}{|c|}{ GR } \\
\hline & $\begin{array}{c}\text { Statistically } \\
\text { Significant } \\
\text { Y/N/NI/NS }\end{array}$ & $\begin{array}{l}\text { Not } \\
\text { Statistically } \\
\text { Significant } \\
\text { Y/N/NI/NS }\end{array}$ & $\begin{array}{l}\text { Statistically } \\
\text { Significant } \\
\text { Y/N/NI/NS }\end{array}$ & $\begin{array}{l}\text { Not } \\
\text { Statistically } \\
\text { Significant } \\
\text { Y/N/NI/NS }\end{array}$ & $\begin{array}{l}\text { Statistically } \\
\text { Significant } \\
\text { Y/N/NI/NS }\end{array}$ & $\begin{array}{c}\text { Not } \\
\text { Statistically } \\
\text { Significant } \\
\text { Y/N/NI/NS }\end{array}$ & $\begin{array}{l}\text { Statistically } \\
\text { Significant } \\
\text { Y/N/NI/NS }\end{array}$ & $\begin{array}{c}\text { Not } \\
\text { Statistically } \\
\text { Significant } \\
\text { Y/N/NI/NS }\end{array}$ & $\begin{array}{l}\text { Statistically } \\
\text { Significant } \\
\text { Y/N/NI/NS }\end{array}$ & $\begin{array}{c}\text { Not } \\
\text { Statistically } \\
\text { Significant } \\
\text { Y/N/NI/NS }\end{array}$ & $\begin{array}{l}\text { Statistically } \\
\text { Significant } \\
\text { Y/N/NI/NS }\end{array}$ & $\begin{array}{c}\text { Not } \\
\text { Statistically } \\
\text { Significant } \\
\text { Y/N/NI/NS }\end{array}$ \\
\hline $\begin{array}{c}\text { Garcia et al., } 2011 \\
\text { (Brazil) [87] }\end{array}$ & $\mathrm{N}$ & $\mathrm{Y}$ & $\mathrm{N}$ & $\mathrm{Y}$ & NS & NS & NS & NS & NS & NS & NS & NS \\
\hline $\begin{array}{c}\text { Joseph et al., } 2014 \\
\text { (India) [88] }\end{array}$ & $Y$ & $\mathrm{~N}$ & Y & $\mathrm{N}$ & $\mathrm{N}$ & Y & $\mathrm{N}$ & Y & $\mathrm{N}$ & Y & NS & NS \\
\hline $\begin{array}{l}\text { Malgikar et al., } 2015 \\
\text { (India) [89] }\end{array}$ & $Y$ & $\mathrm{~N}$ & Y & $\mathrm{N}$ & Y & $\mathrm{N}$ & $\mathrm{Y}$ & $\mathrm{N}$ & $\mathrm{Y}$ & $\mathrm{N}$ & NS & NS \\
\hline $\begin{array}{l}\text { Monzavi et al., } 2016 \\
\text { (Iran) [90] }\end{array}$ & $Y$ & $\mathrm{~N}$ & $\mathrm{~N}$ & $\mathrm{Y}$ & Y & $\mathrm{N}$ & $\mathrm{N}$ & Y & NS & NS & NS & NS \\
\hline $\begin{array}{l}\text { Raj et al., } 2016 \\
\text { (India) [91] }\end{array}$ & $Y$ & $\mathrm{~N}$ & $\mathrm{~N}$ & $\mathrm{Y}$ & NS & NS & Y & $\mathrm{N}$ & Y & $\mathrm{N}$ & NS & NS \\
\hline $\begin{array}{c}\text { Sena et al., } 2019 \\
\text { (Brazil) }[92]\end{array}$ & $\mathrm{N}$ & $\mathrm{Y}$ & $\mathrm{N}$ & $\mathrm{Y}$ & $\mathrm{N}$ & Y & $\mathrm{N}$ & Y & NS & NS & NS & NS \\
\hline $\begin{array}{l}\text { Shingnapurkar et al., } \\
2016 \text { (India) [93] }\end{array}$ & Y & $\mathrm{N}$ & $\mathrm{Y}$ & $\mathrm{N}$ & NS & NS & $\mathrm{N}$ & Y & $\mathrm{N}$ & $\mathrm{Y}$ & NS & NS \\
\hline $\begin{array}{l}\text { Theodoro et al., } 2012 \\
\text { (Brazil) [95] }\end{array}$ & $\mathrm{N}$ & Y & $\mathrm{N}$ & $\mathrm{Y}$ & $\mathrm{N}$ & Y & $\mathrm{N}$ & Y & $\mathrm{N}$ & Y & $\mathrm{N}$ & $\mathrm{Y}$ \\
\hline $\begin{array}{l}\text { Theodoro et al., } 2017 \\
\text { (Brazil) [96] }\end{array}$ & $\mathrm{N}$ & Y & $\mathrm{N}$ & Y & $\mathrm{N}$ & $\mathrm{Y}$ & NS & NS & NS & NS & NS & NS \\
\hline
\end{tabular}




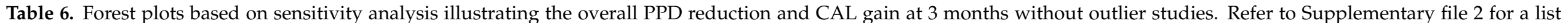
of abbreviations.

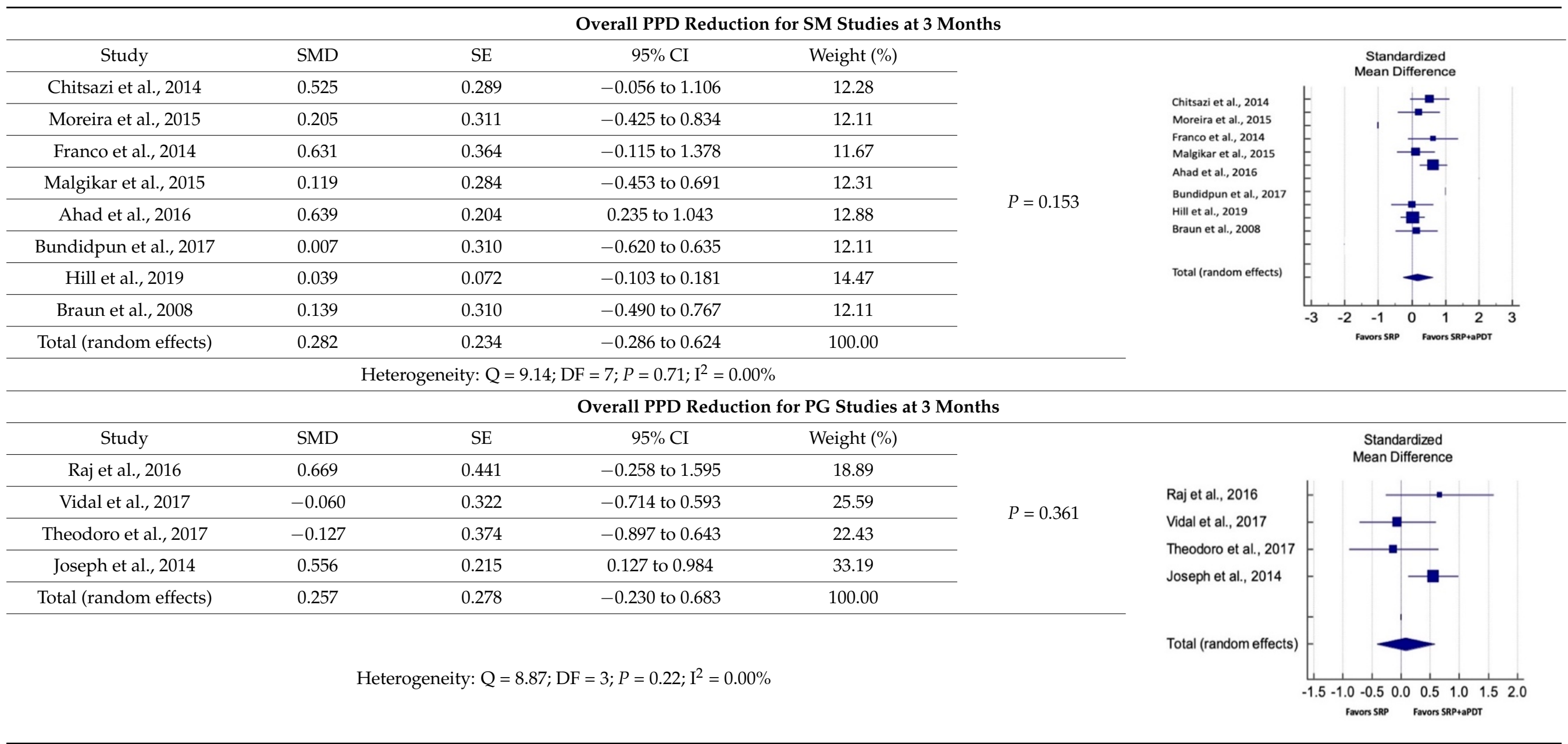


Table 6. Cont.

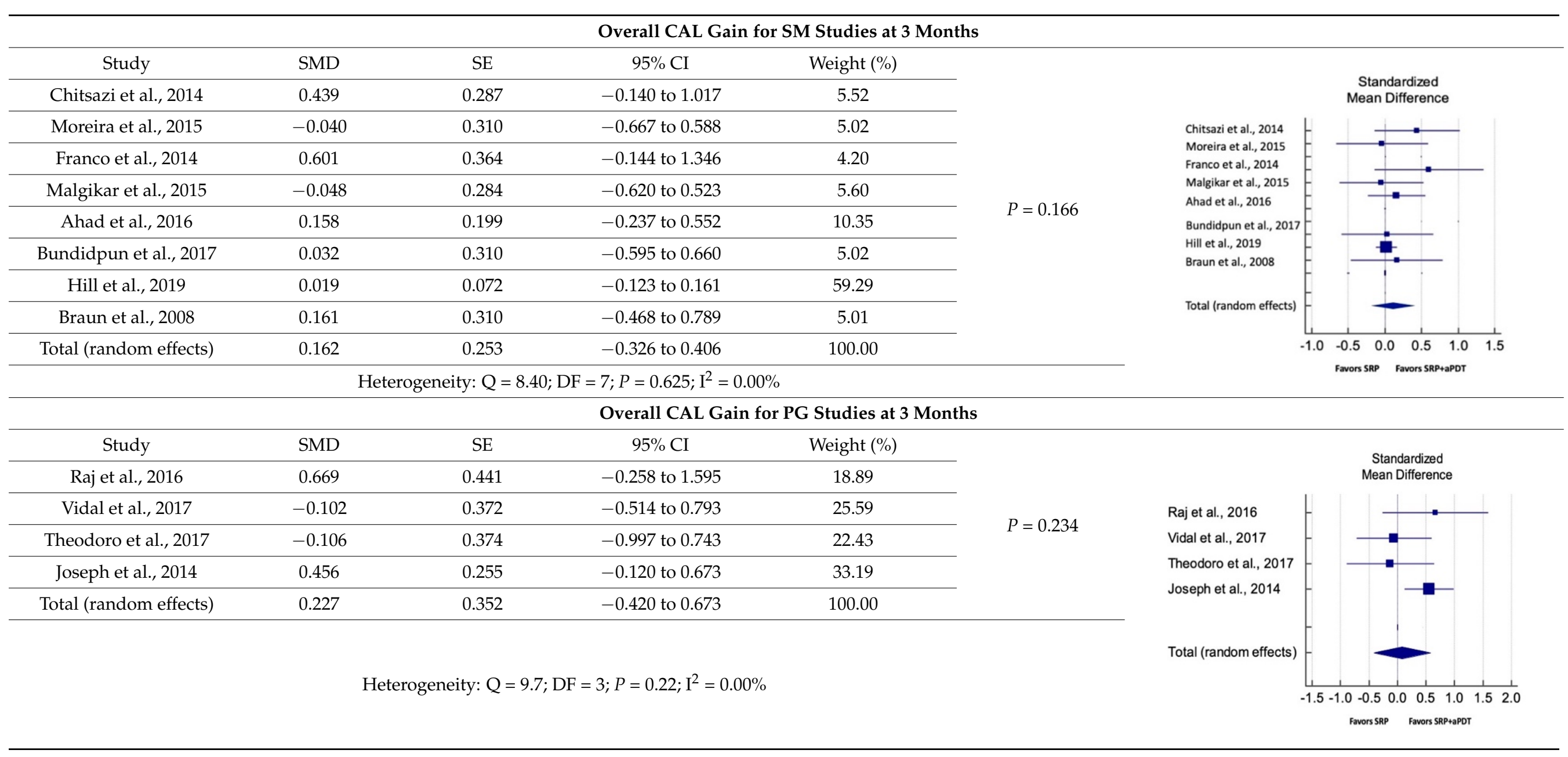




\subsubsection{Publication Bias}

Visual inspection of funnel plots revealed noticeable asymmetry in SM-study analysis for PPD reduction indicating a probable risk of publication bias in SM studies included in this meta-analysis. However, slight asymmetries for PG studies were noted in corresponding funnel plots suggestive of a low risk of publication bias in the same (Figure 6).
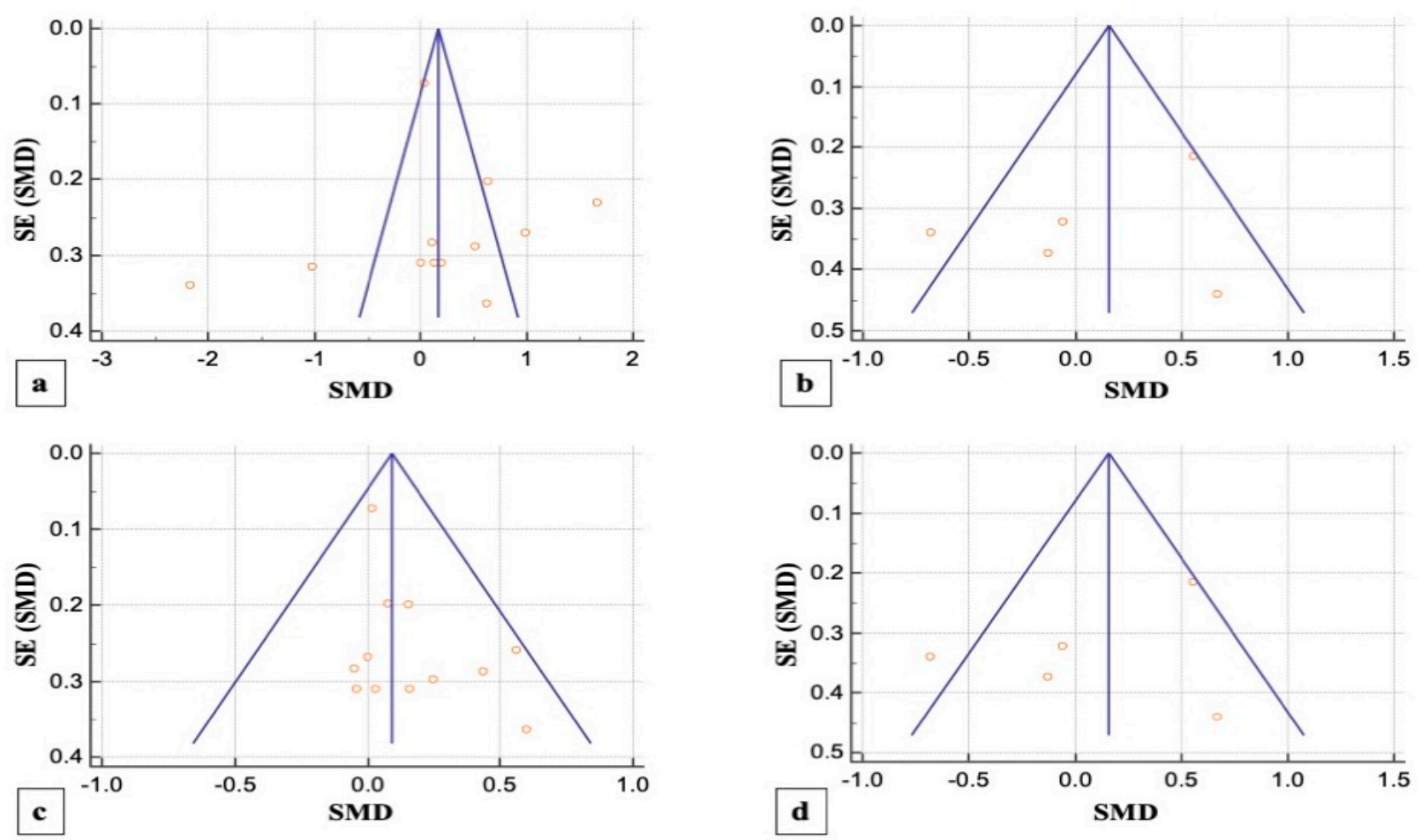

Figure 6. (a,b) Funnel plots illustrating the publication bias in overall PPD reduction in SM and PG studies, respectively; (c,d) funnel plots illustrating the publication bias in overall CAL gain in SM and PG studies, respectively. Each circle represents a single included study, the $y$-axis and $x$-axis represent the standard error of the effect estimate and the results of the study respectively and the graphical plot resembles an inverted funnel with scatter due to sampling variations.

\section{Discussion}

Based on the hypothesis that aPDT monotherapy or as an adjunct to NSPT can enhance the clinical or microbiological or immunological profile in comparison to conventional SRP, or $\mathrm{SRP}+\mathrm{AB}$, a critical appraisal of the available scientific evidence was conducted. After meticulous scrutiny, 31 studies were included in the present systematic review $[2,3,5,17,70-96]$. Owing to the methodological discrepancies, only 18 out 31 studies were eligible for a meta-analysis $[17,71,73-76,80,81,84-86,88-93,96]$. This report is the first to evaluate the role of SRP+aPDT compared to SRP alone or SRP+AB in SM and PG studies in AgP as well as in $\mathrm{CP}$ patients. The results of this meta-analysis indicated that, in comparison to $\mathrm{SRP}$ alone or $\mathrm{SRP}+\mathrm{AB}, \mathrm{SRP}+\mathrm{aPDT}$ failed to show any additional benefit in the management of periodontitis up to six months. A significant heterogeneity was reported, arising from confounders in aPDT protocols. Subsequently, after omitting outlier studies $[74,86,90,92,93]$, sensitivity analysis was able to eliminate heterogeneity completely but failed to report statistically significant improvements in primary outcome variables. Furthermore, risk of publication bias was reported indicating a possible selective outcome reporting in eligible published studies. In some instances, missing outcomes could not be detected by comparing the published report with the respective study protocol due to unavailability of the latter. Until now, seven meta-analyses have been reported to assess the role of aPDT in periodontitis [6-12]. The present review protocol is in accordance with the existing reviews $[8,10-12]$. Azaripour et al. is the only other systematic review 
and meta-analysis that has assessed the efficacy of SRP $+\mathrm{aPDT}$ as compared to SRP alone for SM and PG studies [8]. While three reviews report short-term benefits of aPDT up to 6 months [7-9], four have reported otherwise [6,10-12]. Our findings are in accordance with findings of the latter scientific reports. In order to gain an insight on merits and inadequacies of each included study, a comprehensive and systematic investigation was performed as follows:

\subsection{Role of Baseline Characteristics}

A key feature of RCTs is the application of balanced baseline characteristics in treatment arms of the trial in order to achieve unbiased treatment outcomes [97]. Most often, researchers provide a tabular representation of relevant variables to confirm an impartial baseline evaluation. In case of missing information on baseline characteristics, a 'selection bias' can be suspected [98]. All eligible studies have provided this vital information and were free from any kind of 'selection bias'. Additionally, evidence-based studies have suggested the potential harmful effects of smoking on the onset and progression of periodontitis, for which smokers were excluded $[99,100]$. Likewise, the inter-relationship of periodontitis and its systemic manifestations are well-established, resulting in the exclusion of patients with systemic diseases $[101,102]$. Utilization of 'placebo/sham' procedures to enhance clinical outcomes of the trial is an evidence-based verified concept [103]. In the present systematic review, only six out of 31 studies $[71,78,92-94,96]$ have utilized a 'placebo/sham' procedure as an adjunct to conventional SRP. Furthermore, the role of $\mathrm{SRP}+\mathrm{aPDT}+\mathrm{AB}$, compared to $\mathrm{SRP}+\mathrm{AB}$ and $\mathrm{SRP}+\mathrm{aPDT}$ as well the efficacy of SRP+PS compared to the conventional SRP and SRP+ aPDT have been assessed in this review $[83,95]$. Differences in study designs were apparent and the majority of clinicians have utilized the SM study design in oral health research [104]. Hence, this meta-analysis included both SM and PG studies in order to assess whether the estimated intervention effect has differed between them.

\subsection{Assessment Methods for Various Parameters and Their Inferences to Determine aPDT Efficacy}

A decrease in periodontal inflammation is directly proportional to a decrease in the incidence of BOP and detectable plaque levels [105]. Furthermore, the endpoints of a comprehensive periodontal therapy include PPD reduction and CAL gain, both of which are crucial for determining treatment success [106]. Clinical evidence has proven that there is a direct correlation between initial severity of PPD and CAL values and the amount of post-operative differences [107-109]. In terms of disease severity at baseline evaluation, a lack of homogeneity in pre-treatment values of PPD and CAL in the data extracted from various studies was observed (Table 1). Therefore, variations in level of significance across clinical parameters were noted, thus making it difficult to provide a cumulative result (Table 5). Utilization of a narrow laser optic fibre tip in deep periodontal pockets facilitates easy and atraumatic periodontal pocket probing ultimately resulting in the PPD and GR reduction post-operatively $[3,88]$. Furthermore, evidence-based research has proven that clinical outcome remains unaffected by the type of instrumentation utilized in SRP $[76,110]$, which was observed to vary across all eligible studies (Table 1).

An imbalance amongst the local etiological factors such as dental plaque and calculus, inflammation, and a host defense system can have a great impact on the disease severity and progression [17]. Hence, it is essential to monitor the microbiological and the molecular changes of various growth factors and proinflammatory cytokines. De Oliveira and coworkers have demonstrated, through their studies, the importance of SRP in reducing bacterial load from tooth surface and the failure of aPDT monotherapy in reducing the bacterial counts of A.a. periodontal disease activity as well as bone resorption [2,5].

\subsection{Representation of the Treatment Outcomes}

Positive outcomes of any treatment strategy are governed by several factors such as evaluation of disease status at carefully planned follow-up visits, signs of unevent- 
ful healing, role of supportive periodontal therapy (SPT) and patient compliance with treatment [111-113]. The majority of included studies performed follow-up assessment for up to 3 or 6 months, whereas results of a longer follow-up ranging up to 1-2 years was lacking. Collective data obtained from longitudinal studies have confirmed the role of long-term follow up visits in greater reduction of clinical parameters. These findings have been confirmed by three studies included in the present review $[73,77,95]$. With regard to healing outcomes, 24 out of 31 studies reported no uneventful healing associated with the absence of any postoperative complications, after application of aPDT $[2,3,5,70-75,77-81,83-90,92,95]$. While six studies have failed to provide any information on healing outcomes $[17,76,82,91,93,94]$, one study [96] has reported a gastrointestinal complication in one patient of the aPDT group. This happens to be unique evidence that has not been registered elsewhere. The presence of residual plaque and calculus resulting in a relapse of periodontal disease severity being inevitable with aPDT monotherapy. Hence, the role of SPT is quintessential. In the present systematic review, apart from the three sequential studies conducted by De Oliveira and co-workers $[2,3,5]$ which utilized aPDT monotherapy, all of the other studies have utilized SRP+aPDT. In the former group of studies, a supragingival professional tooth cleaning was performed 14 days prior to the application of aPDT monotherapy in the treatment sites, which have extensively improved the post-operative clinical findings. Likewise, amongst the studies that have assessed the efficacy of SRP+aPDT, only seven studies [71,75,82,84,90,92,95] have mentioned a planned SPT protocol, which was implemented throughout their study period. Furthermore, information on oral hygiene instructions tailored according to respective study criteria has been specified in all included studies. Co-relation of patient's compliance in adhering to hygiene instructions cannot be overlooked, since it is vital for treatment success and prevention of disease recurrence [114]. Quantitative measurement of plaque by means various indices can aid in monitoring compliance towards therapy. In connection to this, the assessment of plaque levels was performed in 21 studies included in this review $[3,17,71,73-75,77-79,81,82,84,85,88-94]$. Therefore, an inconsistency in the representation of treatment outcomes in this review is evident.

\subsection{Role of Laser Parameters}

Apart from study methodology, laser parameters are crucial in determining treatment outcome. Calibration of therapeutic power output with a power-meter can aid in regulating low output power for achieving the desired aPDT effect at treatment sites [115]. This technique can also prevent any inadvertent damage caused by utilization of high output power, resulting in a photothermal effect. However, none of the included studies have utilized a power-meter to calibrate the power output. Furthermore, some other parameters that have been overlooked were: emission mode, contact/non-contact mode, energy/treated site, power output, spot size/fibre diameter, fluence and irradiance. Diode lasers have a high affinity to pigment, which, in the case of aPDT, is the PS. However, inflamed periodontal tissues are rich in blood and high levels of proteins, which are also rich in pigments. Traumatic instrumentation can lacerate the sulcus lining and elicit bleeding [23]. Consequently, the overall aPDT effect, which could be achieved by an effective PS dye-laser wavelength combination, will be compromised [23,116]. Additionally, placement of the fibre tip inside the gingival sulcus needs to be performed judiciously, in order to avoid further trauma to inflamed gingival sulcus, serving as a niche for plaque accumulation and favoring disease relapse $[3,88]$.

Bacterial re-colonization after SRP has been proven to occur after three weeks [117] and, hence, multiple aPDT sessions can help to delay this pathological process. Annaji et al., 2016 [46] have compared the efficacy of three sessions ( 0 , 7th and 21st day) versus a single session ( 0 day) of aPDT in the management of $\mathrm{CP}$, and concluded that the group receiving multiple sessions demonstrated superior treatment outcomes. Nine studies included in this review conducted multiple aPDT sessions $[17,71-75,79,90,96]$, and have unanimously concluded that the utilization of multiple aPDT sessions has positive healing 
effects. However, the frequency of aPDT application varies in all these studies (Table 2). As a result, a conclusion on the ideal number of sessions and frequency of application of aPDT cannot be drawn.

Additionally, PS concentration and pre-irradiation time (wash-out or PS incubation time) are governed by the binding capacity of PS to target cells [118]. It was observed in 13 out of 31 studies, whereby the PS concentration was not reported [5,70,72-74,76,81,84$86,91,93,94]$, whereas a range of PS concentrations have been utilized in the remaining eligible studies. Furthermore, four studies have failed to mention PS pre-irradiation time $[5,75,77,90]$, whereas the remaining 27 studies have reported this time, which ranged from 1-5 min for different PS. An in vitro study by Fumes et al. [119] evaluated the effect of different PS pre-irradiation time periods $(1,2$ and $5 \mathrm{~min}$ ) in aPDT on the biofilms formed by Streptococcus mutans and Candida albicans, by monitoring the microbial load and have successfully demonstrated that the efficacy of all pre-irradiation times was equal. They emphasized patient discomfort associated with longer pre-irradiation times and thus have advised the use of shorter pre-irradiation times $(1 \mathrm{~min})$ in future studies. Up until now, there have been no studies that have determined the minimal duration of PS incubation as well as its role against periopathogens. Hence, further studies on this subject should be sought after. Moreover, discrepancies in the reported data, in terms of the number of sites receiving aPDT application per tooth as well the irradiation time, were noticed amongst the eligible studies (Table 2). These voids have raised concerns regarding the reliability of existing literature, which lacks a reproducible methodology and ultimately hampers the rational use of aPDT in non-surgical management of periodontitis.

\subsection{Role of RoB Assessment}

A vast majority of bias were raised from inadequate randomization, deviations from intended interventions and selective reporting of results (Figures 4 and 5). Another key finding of this systematic review was the presence of a potential conflict of interest in 10 out of the 31 studies $[2,3,5,72-74,76,79,80,94]$. Therefore, the results of the included studies are questionable, and their biased methodology cannot be relied upon.

\subsection{Limitations of the Present Systematic Review}

The majority of the included studies have assessed the efficacy of SRP+aPDT in comparison to aPDT monotherapy resulting in a lack of meta-analysis on the latter. Utilization of a limited number of teeth (mostly anterior teeth) or on specific sites (interproximal sites in case of deep pockets) or on any two quadrants of the dental arch that could facilitate cross contamination from the untreated sites and overshadow the putative benefits of aPDT was noted. In this review, efficacy of aPDT was monitored in systemically healthy non-smokers only, and its benefits in their immunocompromised counterparts were not established. A lack of long-term follow-up in order to determine the stability in healing after aPDT was also observed. The number of studies included in the meta-analysis was nearly half of the studies eligible for a systematic review due to paucity and inconsistency in available literature. Owing to the aforesaid drawbacks, the objective of this review could not be accomplished.

\subsection{Future Scope}

Future investigations should compare aPDT monotherapy versus SRP + aPDT and provide details of all appropriate laser and PS parameters. Efficacy of aPDT in smokers with various systemic diseases in $\mathrm{CP}$ and $\mathrm{AgP}$ should be established. The role of patient related outcomes and SPT should be emphasized upon. Nevertheless, future RCTs must have a robust methodology with balanced baseline characteristics, performed by experienced, masked and calibrated clinicians, which will reduce bias. Owing to the evident benefits of a PG-study design, clinicians should prefer its utilization in order to minimize potential 'carry-over' effects. Additionally, researchers should conduct a full mouth study protocol with a long-term follow-up assessment of minimum 6 months duration, consisting of, but 
not limited to, a vast range of clinical, microbiological, radiographic as well as, immunehistological profiles.

\section{Conclusions}

Within the limits of the present systematic review and meta-analysis, it can be concluded that the efficacy of aPDT in improving treatment outcomes, when it is utilized in the non-surgical management of periodontitis, remains debatable. However, the results of a majority of included studies have demonstrated the effectiveness of aPDT, and this role is more pronounced for SRP+aPDT rather than aPDT monotherapy. A careful and critical appraisal was performed which helped to obtain a qualitative assessment of eligible studies, thus highlighting the substantial flaws that prevent a reproducible methodology. Data on standardized aPDT study protocol, ideal PS dye-laser combination, optimal laser and PS parameters remain inconsistent and inconclusive amongst the prevalent literature owing to a highly inferior RoB in many studies. Finally, future research should aim for well-designed, robust and preferably PG-RCTs that will overcome the abovementioned limitations and confounders, in order to achieve palpable progress in this field of research while ensuring the use of an appropriate local laser safety protocol.

Supplementary Materials: The following are available online at https:/ /www.mdpi.com/article/10 .3390/pharmaceutics13060836/s1, PRISMA Checklist (Supplementary file 1), List of abbreviations (Supplementary file 2).

Author Contributions: Conceptualization, S.D., R.H.; methodology, S.D., R.H.; software, I.R.B., T.S.; validation, S.D., S.B., R.H., I.R.B.; formal analysis, S.B., I.R.B.; investigation, S.D., R.H.; resources, S.D., R.H., S.B., T.S., I.R.B.; data curation, S.D., R.H.; writing: original draft preparation, S.D., R.H.; writing: review and editing, S.D., R.H.; visualization, S.B., I.R.B., T.S.; supervision, S.D., R.H., S.B., T.S., I.R.B.; project administration, T.S., I.R.B.; funding acquisition, T.S. All authors have read and agreed to the published version of the manuscript.

Funding: This research received no external funding.

Institutional Review Board Statement: Not applicable.

Informed Consent Statement: Not applicable.

Conflicts of Interest: The authors declare no conflict of interest.

\section{References}

1. Rajesh, S.; Koshi, E.; Philip, K.; Mohan, A. Antimicrobial photodynamic therapy: An overview. J. Indian Soc. Periodontol. 2011, 15, 323-327. [CrossRef]

2. De Oliveira, R.R.; Schwartz-Filho, H.O.; Novaes, A.B., Jr.; de Souza, R.F.; Taba, M., Jr.; Scombatti de Souza, S.L.; Ribeiro, F.J. Antimicrobial photodynamic therapy in the non-surgical treatment of aggressive periodontitis: Cytokine profile in gingival crevicular fluid, preliminary results. J. Periodontol. 2009, 80, 98-105. [CrossRef] [PubMed]

3. De Oliveira, R.R.; Schwartz-Filho, H.O.; Novaes, A.B., Jr.; Taba, M., Jr. Antimicrobial photodynamic therapy in the non-surgical treatment of aggressive periodontitis: A preliminary randomized controlled clinical study. J. Periodontol. 2007, 78, 965-973. [CrossRef] [PubMed]

4. Van der Velden, U. What exactly distinguishes aggressive from chronic periodontitis: Is it mainly a difference in the degree of bacterial invasiveness? Periodontology 2000 2017, 75, 24-44. [CrossRef] [PubMed]

5. Novaes, A.B., Jr.; Schwartz-Filho, H.O.; de Oliveira, R.R.; Feres, M.; Sato, S.; Figueiredo, L.C. Antimicrobial photodynamic therapy in the non-surgical treatment of aggressive periodontitis: Microbiological profile. Lasers Med. Sci. 2012, 27, 389-395. [CrossRef] [PubMed]

6. Atieh, M.A. Photodynamic therapy as an adjunctive treatment for chronic periodontitis: A meta-analysis. Lasers Med. Sci. 2010, 4, 605-613. [CrossRef] [PubMed]

7. Sgolastra, F.; Petrucci, A.; Severino, M.; Graziani, F.; Gatto, R.; Monaco, A. Adjunctive photodynamic therapy to non-surgical treatment of chronic periodontitis: A systemic review and meta-analysis. J. Clin. Periodontol. 2013, 40, 514-526. [CrossRef] [PubMed]

8. Azaripour, A.; Dittrich, S.; Van Noorden, C.J.F.; Willershausen, C. Efficacy a photodynamic therapy as adjunct treatment of chronic periodontitis: A systematic review and meta-analysis. Lasers Med. Sci. 2018, 33, 407-423. [CrossRef]

9. Sgolastra, F.; Petrucci, A.; Gatto, R.; Mazzo, G.; Monaco, A. Photodynamic therapy in the treatment of chronic periodontitis: A systematic review and meta-analysis. Lasers Med. Sci. 2013, 28, 669-682. [CrossRef] 
10. Souza, E.; Medeiros, A.C.; Gurgel, B.C.; Sarmento, C. Antimicrobial photodynamic therapy in the treatment of aggressive periodontitis: A systematic review and meta-analysis. Lasers Med. Sci. 2016, 31, 187-196. [CrossRef] [PubMed]

11. Azarpazhooh, A.; Shah, P.S.; Tenenbaum, H.C.; Goldberg, M.B. The effect of photodynamic therapy for periodontitis: A systematic review and meta-analysis. J. Periodontol. 2010, 81, 4-14. [CrossRef]

12. Akram, Z.; Hyder, T.; Al-Hammoudi, N.; Binshabaib, M.S.; Alharthi, S.S.; Hanif, A. Efficacy of photodynamic therapy versus antibiotics as an adjunct to scaling and root planing in the treatment of periodontitis: A systematic review and meta-analysis. Photodiagnosis Photodyn. Ther. 2017, 19, 86-92. [CrossRef] [PubMed]

13. Vohra, F.; Akram, Z.; Safii, S.H.; Vaithilingam, R.D.; Ghanem, A.; Sergis, K.; Javed, F. Role of antimicrobial photodynamic therapy in the treatment of aggressive periodontitis: A systematic review. Photodiagnosis Photodyn. Ther. 2016, 13, 139-147. [CrossRef] [PubMed]

14. Chatzopoulos, G.S.; Doufexi, A.E. Photodynamic therapy in the treatment of aggressive periodontitis: A systematic review. Med. Oral Patol. Oral Cir. Bucal 2016, 21, e192-e200. [CrossRef] [PubMed]

15. Reddy, P.; Reddy, C.; Krishna Kumar, R.V.S.; Sudhir, K.M.; Srinivasulu, G. Effect of photodynamic therapy on aggressive periodontitis-A systematic review. Int. J. Sci. Res. Manag. 2017, 5, 7177-7185.

16. Pal, A.; Paul, S.; Perry, R.; Puryer, J. Is the use of antimicrobial photodynamic therapy or systemic antibiotics more effective in improving periodontal health when used in conjunction with localised non-surgical periodontal therapy? A systematic review. Dent. J. 2019, 7, 108. [CrossRef]

17. Franco, E.J.; Pogue, R.E.; Sakamoto, L.H.; Cavalcante, L.L.; Carvalho, D.R.; de Andrade, R.V. Increased expression of genes after periodontal treatment with photodynamic therapy. Photodiagnosis Photodyn. Ther. 2014, 11, 41-47. [CrossRef] [PubMed]

18. Van Assche, N.; Van Essche, M.; Pauwels, M.; Teughels, W.; Quirynen, M. Do periodontopathogens disappear after full-mouth tooth extraction? J. Clin. Periodontol. 2009, 36, 1043-1047. [CrossRef]

19. Guerrero, A.; Griffiths, G.S.; Nibali, L.; Suvan, J.; Moles, D.R.; Laurell, L.; Tonetti, M.S. Adjunctive benefits of systemic amoxicillin and metronidazole in non-surgical treatment of generalized aggressive periodontitis: A randomized placebo-controlled clinical trial. J. Clin. Periodontol. 2005, 32, 1096-1107. [CrossRef]

20. Sigusch, B.; Beier, M.; Klinger, G.; Pfister, W.; Glockmann, E. A 2-step non-surgical procedure and systemic antibiotics in the treatment of rapidly progressive periodontitis. J. Periodontol. 2001, 72, 275-283. [CrossRef]

21. Herrera, D.; Sanz, M.; Jepsen, S.; Needleman, I.; Roldán, S. A systematic review on the effect of systemic antimicrobials as an adjunct to scaling and root planing in periodontitis patients. J. Clin. Periodontol. 2002, 29, 136-162. [CrossRef]

22. Pallasch, T.J. Antibiotic resistance. Dent. Clin. N. Am. 2003, 47, 623-639. [CrossRef]

23. Andersen, R.C.; Loebel, N.G. Photodynamic disinfection in the treatment of chronic adult periodontitis: A multicenter clinical trial. J. Dent. Health Oral Disord. Ther. 2017, 8, 00289. [CrossRef]

24. Moher, D.; Liberati, A.; Tetzlaff, J.; Altman, D.G.; PRISMA Group. Preferred reporting items for systematic reviews and meta-analyses: The PRISMA statement. BMJ 2009, 339, b2535. [CrossRef] [PubMed]

25. Higgins, J.P.T.; Green, S. Cochrane Handbook for Systematic Reviews of Interventions Version 5.1.0 The Cochrane Collaboration. 2011. Available online: http:/ / www.cochrane-handbook.org (accessed on 12 February 2021).

26. Armitage, G.C. Development of a classification system for periodontal diseases and conditions. Ann. Periodontol. 1999, 4, 1-6. [CrossRef] [PubMed]

27. McHugh, M.L. Inter-rate reliability: The kappa statistic. Biochem. Med. 2012, 22, 276-282. [CrossRef]

28. Sterne, J.A.C.; Savović, J.; Page, M.J.; Elbers, R.G.; Blencowe, N.S.; Boutron, I.; Cates, C.J.; Cheng, H.Y.; Corbett, M.S.; Eldridge, S.M.; et al. RoB 2: A revised tool for assessing risk of bias in randomised trials. BMJ 2019, 366, 14898. [CrossRef] [PubMed]

29. Altman, D.G.; Schulz, K.F.; Moher, D.; Egger, M.; Davidoff, F.; Elbourne, D.; Gøtzsche, P.C.; Lang, T.; CONSORT GROUP (Consolidated Standards of Reporting Trials). The revised CONSORT statement for reporting randomized trials: Explanation and elaboration. Ann. Intern Med. 2001, 134, 663-694. [CrossRef]

30. Higgins, J.P.T.; Eldridge, S.; Li, T. Chapter 23: Including variants on randomized trials. In Cochrane Handbook for Systematic Reviewws of Interventions Version 6.0; Higgins, J.P.T., Thomas, J., Chandler, J., Cumpston, M., Li, T., Page, M.J., Welch, V.A., Eds.; (updated July 2019); Cochrane: Oxford, UK, 2019. Available online: www.training.cochrane.org/handbook (accessed on 12 February 2021).

31. Hozo, S.P.; Djulbegovic, B.; Hozo, I. Estimating the mean and variance from the median, range, and the size of a sample. BMC Med. Res. Methodol. 2005, 5, 13. [CrossRef]

32. Lau, J.; Ioannidis, J.P.; Schmid, C.H. Quantitative synthesis in systematic reviews. Ann. Intern Med. 1997, 127, 820-826. [CrossRef] [PubMed]

33. Higgins, J.P.T.; Thompson, S.G. Quantifying heterogeneity in a meta-analysis. Stat. Med. 2002, 21, 1539-1558. [CrossRef] [PubMed]

34. Sterne, J.A.C.; Egger, M. Funnel plots for detecting bias in meta-analysis: Guidelines on choice of axis. J. Clin. Epidemiol. 2001, 54, 1046-1055. [CrossRef]

35. Theodoro, L.H.; Assem, N.Z.; Longo, M.; Alves, M.L.F.; Duque, C.; Stipp, R.N.; Vizoto, N.L.; Garcia, V.G. Treatment of periodontitis in smokers with multiple sessions of antimicrobial photodynamic therapy or systemic antibiotics: A randomized clinical trial. Photodiagnosis Photodyn. Ther. 2018, 22, 217-222. [CrossRef] [PubMed]

36. Al-Zahrani, M.S.; Austah, O.N. Photodynamic therapy as an adjunctive to scaling and root planing in treatment of chronic periodontitis in smokers. Saudi Med. J. 2011, 32, 1183-1188. [PubMed] 
37. Tabenski, L.; Moder, D.; Cieplik, F.; Schenke, F.; Hiller, K.A.; Buchalla, W.; Schmalz, G.; Christgau, M. Antimicrobial photodynamic therapy vs. local minocycline in addition to non-surgical therapy of deep periodontal pockets: A controlled randomized clinical trial. Clin. Oral Investig. 2017, 21, 2253-2264. [CrossRef] [PubMed]

38. Ge, L.; Shu, R.; Li, Y.; Li, C.; Luo, L.; Song, Z.; Xie, Y.; Liu, D. Adjunctive effect of photodynamic therapy to scaling and root planing in the treatment of chronic periodontitis. Photomed. Laser Surg. 2011, 29, 33-37. [CrossRef] [PubMed]

39. Loebel, N.G.; Galler, C.C.; Andersen, R.C. Antimicrobial photodynamic therapy in the treatment of chronic adult periodontitis. Oral Health Dental Sci. 2018, 2, 1-7. [CrossRef]

40. Harmouche, L.; Courval, A.; Mathieu, A.; Peti, C.; Huck, O.; Severac, F.; Davideau, J.L. Impact of tooth-related factors on photodynamic therapy effectiveness during active periodontal therapy: A 6-months split-mouth randomized clinical trial. Photodiagnosis Photodyn. Ther. 2019, 27, 167-172. [CrossRef] [PubMed]

41. Christodoulides, N.; Nikolidakis, D.; Chondros, P.; Becker, J.; Schwarz, F.; Rossler, R.; Sculean, A. Photodynamic therapy as an adjunct to non-surgical periodontal treatment: A randomized, controlled clinical trial. J. Periodontol. 2008, 79, 1638-1644. [CrossRef]

42. Polansky, R.; Haas, M.; Heschl, A.; Wimmer, G. Clinical effectiveness of photodynamic therapy in the treatment of periodontitis. J. Clin. Periodontol. 2009, 36, 575-580. [CrossRef]

43. Badea, M.E.; Serbanescu, A.; Hedesiu, M.; Badea, A.F. Photodynamic Laser therapy in patients with periodontitis. TMJ 2010, 60, 18-22.

44. Andersen, R.; Loebel, N.; Hammond, D.; Wilson, M. Treatment of periodontal disease by photodisinfection compared to scaling and root planing. J. Clin. Dent. 2007, 18,34-38.

45. Alwaeli, H.A.; Al-Khateeb, S.N.; Al-Sadi, A. Long-term clinical effect of adjunctive antimicrobial photodynamic therapy in periodontal treatment: A randomized clinical trial. Lasers Med. Sci. 2015, 30, 801-807. [CrossRef] [PubMed]

46. Annaji, S.; Sarkar, I.; Rajan, P.; Pai, J.; Malagi, S.; Bharmappa, R.; Kamath, V. Efficacy of photodynamic therapy and lasers as an adjunct to scaling and root planing in the treatment of aggressive periodontitis-A clinical and microbiologic short term study. J. Clin. Diagn. Res. 2016, 10, ZC08-ZC12. [CrossRef] [PubMed]

47. Dilsiz, A.; Canakci, V.; Aydin, T. Clinical effects of potassium-titanyl-phosphate laser and photodynamic therapy on outcomes of treatment of chronic periodontitis: A randomized controlled clinical trial. J. Periodontol. 2013, 84, 278-286. [CrossRef] [PubMed]

48. Lui, J.; Corbet, E.F.; Jin, L. Combined photodynamic and low-level laser therapies as an adjunct to nonsurgical treatment of chronic periodontitis. J. Periodontal Res. 2011, 46, 89-96. [CrossRef] [PubMed]

49. Mistry, A.; Pereira, R.; Kini, V.; Padhye, A. Effect of combined therapy using diode laser and photodynamic therapy on levels of IL-17 in gingival crevicular fluid in patients with chronic periodontitis. J. Lasers Med. Sci. 2016, 7, 250-255. [CrossRef] [PubMed]

50. Srikanth, K.; Chandra, R.V.; Reddy, A.A.; Reddy, B.H.; Reddy, C.; Naveen, A. Effect of a single session of antimicrobial photodynamic therapy using indocyanine green in the treatment of chronic periodontitis: A randomized controlled pilot trial. Quintessence Int. 2015, 46, 391-400. [CrossRef] [PubMed]

51. Grzech-Leśniak, K.; Matys, J.; Dominiak, M. Comparison of the clinical and microbiological effects of antibiotic therapy in periodontal pockets following laser treatment: An in vivo study. Adv. Clin. Exp. Med. 2018, 27, 1263-1270. [CrossRef] [PubMed]

52. Kamma, J.J.; Vasdekis, V.G.; Romanos, G.E. The effect of diode laser $(980 \mathrm{~nm})$ treatment on aggressive periodontitis: Evaluation of microbial and clinical parameters. Photomed. Laser Surg. 2009, 27, 11-19. [CrossRef]

53. Matarese, G.; Ramaglia, L.; Cicciù, M.; Cordasco, G.; Isola, G. The effects of diode laser therapy as an adjunct to scaling and root planing in the treatment of aggressive periodontitis: A 1-year randomized controlled clinical trial. Photomed. Laser Surg. 2017, 35, 702-709. [CrossRef] [PubMed]

54. Annaji, S.; Sarkar, I.; Rajan, P.; Pai, J.; Malagi, S.; Kamath, V.; Barmappa, R. Comparative evaluation of the efficacy of curcumin gel with and without photo activation as an adjunct to scaling and root planing in the treatment of chronic periodontitis: A split mouth clinical and microbiological study. J. Nat. Sci. Biol. Med. 2015, 6, S102-S109. [CrossRef]

55. Borekci, T.; Meseli, S.E.; Noyan, U.; Kuru, B.E.; Kuru, L. Efficacy of adjunctive photodynamic therapy in the treatment of generalized aggressive periodontitis: A randomized controlled clinical trial. Lasers Surg. Med. 2019, 51, 167-175. [CrossRef] [PubMed]

56. Pulikkotil, S.J.; Toh, C.G.; Mohandas, K.; Leong, K. Effect of photodynamic therapy adjunct to scaling and root planing in periodontitis patients: A randomized clinical trial. Aust. Dent. J. 2016, 61, 440-445. [CrossRef]

57. Saitawee, D.; Teerakapong, A.; Morales, N.P.; Jitprasertwong, P.; Hormdee, D. Photodynamic therapy of Curcuma longa extract stimulated with blue light against Aggregatibacter actinomycetemcomitans. Photodiagnosis Photodyn. Ther. 2018, 22, 101-105. [CrossRef]

58. Bassir, S.H.; Moslemi, N.; Jamali, R.; Mashmouly, S.; Fekrazad, R.; Chiniforush, N.; Shamshiri, A.R.; Nowzari, H. Photoactivated disinfection using light-emitting diode as an adjunct in the management of chronic periodontitis: A pilot double-blind split-mouth randomized clinical trial. J. Clin. Periodontol. 2013, 40, 65-72. [CrossRef] [PubMed]

59. Husejnagic, S.; Lettner, S.; Laky, M.; Georgopoulos, A.; Moritz, A.; Rausch-Fan, X. Photoactivated disinfection in periodontal treatment: A randomized controlled clinical split-mouth trial. J. Periodontol. 2019, 90, 1260-1269. [CrossRef] [PubMed]

60. Maria, A.M.; Ursarescu, I.G.; Solomon, S.; Foia, L. Evaluation the effects of led photo-activated disinfection on periodontal clinical parameters in patients with chronic periodontitis. Balk. J. Dent. Med. 2016, 20, 29-32. [CrossRef] 
61. Campos, G.N.; Pimentel, S.P.; Ribeiro, F.V.; Casarin, R.C.; Cirano, F.R.; Saraceni, C.H.; Casati, M.Z. The adjunctive effect of photodynamic therapy for residual pockets in single- rooted teeth: A randomized controlled clinical trial. Lasers Med. Sci. 2013, 28, 317-324. [CrossRef] [PubMed]

62. Corrêa, M.G.; Oliveira, D.H.; Saraceni, C.H.; Ribeiro, F.V.; Pimentel, S.P.; Cirano, F.R.; Casarin, R.C. Short-term microbiological effects of photodynamic therapy in non-surgical periodontal treatment of residual pockets: A split-mouth RCT. Lasers Surg. Med. 2016, 48, 944-950. [CrossRef] [PubMed]

63. Petelin, M.; Perkič, K.; Seme, K.; Gašpirc, B. Effect of repeated adjunctive antimicrobial photodynamic therapy on subgingival periodontal pathogens in the treatment of chronic periodontitis. Lasers Med. Sci. 2015, 30, 1647-1656. [CrossRef] [PubMed]

64. Cappuyns, I.; Cionca, N.; Wick, P.; Giannopoulou, C.; Mombelli, A. Treatment of residual pockets with photodynamic therapy, diode laser, or deep scaling. A randomized, split-mouth controlled clinical trial. Lasers Med. Sci. 2012, 27, 979-986. [CrossRef] [PubMed]

65. Lulic, M.; Leiggener, G.I.; Salvi, G.E.; Ramseier, C.A.; Mattheos, N.; Lang, N.P. One-year outcomes of repeated adjunctive photodynamic therapy during periodontal maintenance: A proof-of-principle randomized-controlled clinical trial. J. Clin. Periodontol. 2009, 36, 661-666. [CrossRef] [PubMed]

66. Rühling, A.; Fanghänel, J.; Houshmand, M.; Kuhr, A.; Meisel, P.; Schwahn, C.; Kocher, T. Photodynamic therapy of persistent pockets in maintenance patients-A clinical study. Clin. Oral Investig. 2010, 14, 637-644. [CrossRef]

67. Barbosa, F.I.; Araújo, P.V.; Machado, L.J.C.; Magalhães, C.S.; Guimarães, M.M.; Moreira, A.N. Effect of photodynamic therapy as an adjuvant to non-surgical periodontal therapy: Periodontal and metabolic evaluation in patients with type 2 diabetes mellitus. Photodiagnosis Photodyn. Ther. 2018, 22, 245-250. [CrossRef] [PubMed]

68. Ramos, U.D.; Ayub, L.G.; Reino, D.M.; Grisi, M.F.; Taba, M., Jr.; Souza, S.L.; Palioto, D.B.; Novaes, A.B., Jr. Antimicrobial photodynamic therapy as an alternative to systemic antibiotics: Results from a double-blind, randomized, placebo-controlled, clinical study on type 2 diabetics. J. Clin. Periodontol. 2016, 43, 147-155. [CrossRef]

69. Alvarenga, L.H.; Gomes, A.C.; Carribeiro, P.; Godoy-Miranda, B.; Noschese, G.; Simões Ribeiro, M.; Kato, I.T.; Bussadori, S.K.; Pavani, C.; Geraldo, Y.G.; et al. Parameters for antimicrobial photodynamic therapy on periodontal pocket- Randomized clinical trial. Photodiagnosis Photodyn. Ther. 2019, 27, 132-136. [CrossRef] [PubMed]

70. Pourabbas, R.; Kashefimehr, A.; Rahmanpour, N.; Babaloo, Z.; Kishen, A.; Tenenbaum, H.C.; Azarpazhooh, A. Effects of photodynamic therapy on clinical and gingival crevicular fluid inflammatory biomarkers in chronic periodontitis: A split-mouth randomized clinical trial. J. Periodontol. 2014, 85, 1222-1229. [CrossRef] [PubMed]

71. Moreira, A.L.; Novaes, A.B., Jr.; Grisi, M.F.; Taba, M., Jr.; Souza, S.L.; Palioto, D.B.; de Oliveira, P.G.; Casati, M.Z.; Casarin, R.C.; Messora, M.R. Antimicrobial photodynamic therapy as an adjunct to non-surgical treatment of aggressive periodontitis: A split-mouth randomized controlled trial. J. Periodontol. 2015, 86, 376-386. [CrossRef]

72. Skurska, A.; Dolinska, E.; Pietruska, M.; Pietruski, J.K.; Dymicka, V.; Kemona, H.; Arweiler, N.B.; Milewsk, R.; Sculean, A. Effect of nonsurgical periodontal treatment in conjunction with either systemic administration of amoxicillin and metronidazole or additional photodynamic therapy on the concentration of matrix metalloproteinases 8 and 9 in gingival crevicular fluid in patients with aggressive periodontitis. BMC Oral Health 2015, 15, 63. [CrossRef]

73. Arweiler, N.B.; Pietruska, M.; Pietruski, J.; Skurska, A.; Dolińsk, E.; Heumann, C.; Auschill, T.M.; Sculean, A. Six-month results following treatment of aggressive periodontitis with antimicrobial photodynamic therapy or amoxicillin and metronidazole. Clin. Oral Investig. 2014, 18, 2129-2135. [CrossRef] [PubMed]

74. Arweiler, N.B.; Pietruska, M.; Skurska, A.; Dolińska, E.; Pietruski, J.K.; Bläs, M.; Auschill, T.M.; Sculean, A. Nonsurgical treatment of aggressive periodontitis with photodynamic therapy or systemic antibiotics. Three-month results of a randomized, prospective, controlled clinical study. Schweiz. Monatsschr. Zahnmed. 2013, 123, 532-544. [PubMed]

75. Segarra-Vidal, M.; Guerra-Ojeda, S.; Vallés, L.S.; López-Roldán, A.; Mauricio, M.D.; Aldasoro, M.; Alpiste-Illueca, F.; Vila, J.M. Effects of photodynamic therapy in periodontal treatment: A randomized, controlled clinical trial. J. Clin. Periodontol. 2017, 44, 915-925. [CrossRef] [PubMed]

76. Braun, A.; Dehn, C.; Krause, F.; Jepsen, S. Short-term clinical effects of adjunctive antimicrobial photodynamic therapy in periodontal treatment: A randomized clinical trial. J. Clin. Periodontol. 2008, 35, 877-884. [CrossRef] [PubMed]

77. Berakdar, M.; Callaway, A.; Eddin, M.F.; Ross, A.; Willershausen, B. Comparison between scaling-root-planing (SRP) and SRP/Photodynamic therapy: Six-month study. Head Face Med. 2012, 8, 12. [CrossRef]

78. Raut, C.P.; Sethi, K.S.; Kohale, B.R.; Mamajiwala, A.; Warang, A. Indocyanine green- mediated photothermal therapy in treatment of chronic periodontitis: A clinico- microbiological study. J. Indian Soc. Periodontol. 2018, 22, 221-227. [CrossRef]

79. Hokari, T.; Morozumi, T.; Komatsu, Y.; Shimizu, T.; Yoshino, T.; Tanaka, M.; Tanaka, Y.; Nohno, K.; Kubota, T.; Yoshie, H. Effects of antimicrobial photodynamic therapy and local administration of minocycline on clinical, microbiological, and inflammatory markers of periodontal pockets: A Pilot Study. Int. J. Dent. 2018, 1748584. [CrossRef] [PubMed]

80. Hill, G.; Dehn, C.; Hinze, A.V.; Frentzen, M.; Meister, J. Indocyanine green-based adjunctive antimicrobial photodynamic therapy for treating chronic periodontitis: A randomized clinical trial. Photodiagnosis Photodyn. Ther. 2019, 26, 29-35. [CrossRef]

81. Ahad, A.; Lamba, A.K.; Faraz, F.; Tandon, S.; Chawla, K.; Yadav, N. Effect of antimicrobial photodynamic therapy as an adjunct to nonsurgical treatment of deep periodontal pockets: A clinical study. J. Lasers Med. Sci. 2016, 7, 220-226. [CrossRef] 
82. Balata, M.L.; Andrade, L.P.; Santos, D.B.; Cavalcanti, A.N.; Tunes Uda, R.; Ribeiro Édel, P.; Bittencourt, S. Photodynamic therapy associated with full-mouth ultrasonic debridement in the treatment of severe chronic periodontitis: A randomized-controlled clinical trial. J. Appl. Oral Sci. 2013, 21, 208-214. [CrossRef] [PubMed]

83. Bechara Andere, N.M.R.; Dos Santos, N.C.C.; Araujo, C.F.; Mathias, I.F.; Rossato, A.; de Marco, A.C.; Santamaria, M., Jr.; Jardini, M.A.N.; Santamaria, M.P. Evaluation of the local effect of nonsurgical periodontal treatment with and without systemic antibiotic and photodynamic therapy in generalized aggressive periodontitis. A randomized clinical trial. Photodiagnosis Photodyn. Ther. 2018, 24, 115-120. [CrossRef] [PubMed]

84. Bundidpun, P.; Srisuwantha, R.; Laosrisin, N. Clinical effects of photodynamic therapy as an adjunct to full-mouth ultrasonic scaling and root planing in treatment of chronic periodontitis. Laser Ther. 2018, 27, 33-39. [CrossRef] [PubMed]

85. Chitsazi, M.T.; Shirmohammadi, A.; Pourabbas, R.; Abolfazli, N.; Farhoudi, I.; Azar, B.D.; Farhadi, F. Clinical and microbiological effects of photodynamic therapy associated with non- surgical treatment in aggressive periodontitis. J. Dent. Res. Dent. Clin. Dent. Prospects 2014, 8, 153-159. [CrossRef] [PubMed]

86. Chitsazi, M.T.; Kashefimehr, A.; Pourabbas, R.; Shirmohammadi, A.; Ghasemi Barghi, V.; Daghigh Azar, B. Efficacy of subgingival application of xanthan-based chlorhexidine gel adjunctive to full-mouth root planing assessed by real-time PCR: A Microbiologic and Clinical Study. J. Dent. Res. Dent. Clin. Dent. Prospects 2013, 7, 95-101. [CrossRef] [PubMed]

87. Garcia, F.B.; Dias, A.T.; Tinoco, B.E.M.; Fischeer, R.G. Evaluation of the efficacy of photodynamic therapy as adjunct to periodontal treatment in patients with aggressive periodontitis. Rev. Periodontia 2011, 21, 12-19.

88. Betsy, J.; Prasanth, C.S.; Baiju, K.V.; Prasanthila, J.; Subhash, N. Efficacy of antimicrobial photodynamic therapy in the management of chronic periodontitis: A randomized controlled clinical trial. J. Clin. Periodontol. 2014, 4, 573-581. [CrossRef] [PubMed]

89. Malgikar, S.; Reddy, S.H.; Babu, P.R.; Sagar, S.V.; Kumar, P.S.; Reddy, G.J. A randomized controlled clinical trial on efficacy of photodynamic therapy as an adjunct to nonsurgical treatment of chronic periodontitis. J. Dent. Lasers 2015, 9, 75-79. [CrossRef]

90. Monzavi, A.; Chinipardaz, Z.; Mousavi, M.; Fekrazad, R.; Moslemi, N.; Azaripour, A.; Bagherpasand, O.; Chiniforush, N. Antimicrobial photodynamic therapy using diode laser activated indocyanine green as an adjunct in the treatment of chronic periodontitis: A randomized clinical trial. Photodiagnosis Photodyn. Ther. 2016, 14, 93-97. [CrossRef] [PubMed]

91. Raj, K.R.; Musalaiah, S.; Nagasri, M.; Kumar, P.A.; Reddy, P.I.; Greeshma, M. Evaluation of efficacy of photodynamic therapy as an adjunct to nonsurgical periodontal therapy in treatment of chronic periodontitis patients: A clinico-microbiological study. Indian J. Dent. Res. 2016, 27, 483-487. [CrossRef] [PubMed]

92. Sena, I.A.A.; Silva, D.N.A.; Azevedo, M.L.D.S.; da Silva, N.T.; Longo, J.P.F.; de Moraes, M.; de Aquino Martins, A.R.L. Antimicrobial photodynamic therapy using a chloro-aluminum phthalocyanine adjuvant to nonsurgical periodontal treatment does not improve clinical parameters in patients with chronic periodontitis. Photobiomodul. Photomed. Laser Surg. 2019, 37, 729-735. [CrossRef]

93. Shingnapurkar, S.H.; Mitra, D.K.; Kadav, M.S.; Shah, R.A.; Rodrigues, S.V.; Prithyani, S.S. The effect of indocyanine greenmediated photodynamic therapy as an adjunct to scaling and root planing in the treatment of chronic periodontitis: A comparative split-mouth randomized clinical trial. Indian J. Dent. Res. 2016, 27, 609-617. [CrossRef] [PubMed]

94. Sigusch, B.W.; Engelbrecht, M.; Völpel, A.; Holletschke, A.; Pfister, W.; Schütze, J. Full-mouth antimicrobial photodynamic therapy in Fusobacterium nucleatum-infected periodontitis patients. J. Periodontol. 2010, 81, 975-981. [CrossRef] [PubMed]

95. Theodoro, L.H.; Silva, S.P.; Pires, J.R.; Soares, G.H.G.; Pontes, A.E.F.; Zuza, E.P.; Spolidório, D.M.; de Toledo, B.E.; Garcia, V.G. Clinical and microbiological effects of photodynamic therapy associated with nonsurgical periodontal treatment: A 6-month follow-up. Lasers Med. Sci. 2012, 27, 687-693. [CrossRef]

96. Theodoro, L.H.; Lopes, A.B.; Nuernberg, M.A.A.; Cláudio, M.M.; Miessi, D.M.J.; Alves, M.L.F.; Duque, C.; Mombelli, A.; Garcia, V.G. Comparison of repeated applications of aPDT with amoxicillin and metronidazole in the treatment of chronic periodontitis: A short-term study. J. Photochem. Photobiol. B 2017, 174, 364-369. [CrossRef] [PubMed]

97. Festic, E.; Rawal, B.; Gajic, O. How to improve assessment of balance in baseline characteristics of clinical trial participantsexample from PROSEVA trial data? Ann. Transl. Med. 2016, 4, 79. [CrossRef] [PubMed]

98. Tripepi, G.; Jager, K.J.; Dekker, F.W.; Zoccali, C. Selection bias and information bias in clinical research. Nephron. Clin. Pract. 2010, 115, c94-c99. [CrossRef] [PubMed]

99. Johnson, G.K.; Hill, M. Cigarette smoking and the periodontal patient. J. Periodontol. 2004, 75, 196-209. [CrossRef]

100. Leite, F.R.M.; Nascimento, G.G.; Scheutz, F.; López, R. Effect of smoking on Periodontitis: A Systematic Review and Metaregression. Am. J. Prev. Med. 2018, 54, 831-841. [CrossRef]

101. Arigbede, A.O.; Babatope, B.O.; Bamidele, M.K. Periodontitis and systemic diseases: A literature review. J. Indian Soc. Periodontol. 2012, 16, 487-491. [CrossRef] [PubMed]

102. Komine-Aizawa, S.; Aizawa, S.; Hayakawa, S. Periodontal diseases and adverse pregnancy outcomes. J. Obstet. 2019, 45, 5-12. [CrossRef]

103. Blasini, M.; Movsas, S.; Colloca, L. Placebo hypoalgesic effects in pain: Potential applications in dental and orofacial pain management. Semin. Orthod. 2018, 24, 259-268. [CrossRef] [PubMed]

104. Smail-Faugeron, V.; Fron-Chabouis, H.; Courson, F.; Durieux, P. Erratum to: Comparison of intervention effects in split-mouth and parallel-arm randomized controlled trials: A meta-epidemiological study. BMC Med. Res. Methodol. 2015, 15, 72. [CrossRef] [PubMed] 
105. Lang, N.P.; Joss, A.; Orsanic, T.; Gusberti, F.A.; Siegrist, B.E. Bleeding on probing. A predictor for the progression of periodontal disease? J. Clin. Periodontol. 1986, 13, 590-596. [CrossRef] [PubMed]

106. Greenstein, G. Contemporary interpretation of probing depth assessments: Diagnostic and therapeutic implications. A literature review. J. Periodontol. 1997, 68, 1194-1205. [CrossRef]

107. Ramfjord, S.P.; Caffesse, R.G.; Morrison, E.C.; Hill, R.W.; Kerry, G.J.; Appleberry, E.A.; Nissle, R.R.; Stults, D.L. 4 modalities of periodontal treatment compared over 5 years. J. Clin. Periodontol. 1987, 14, 445-452. [CrossRef]

108. Kaldahl, W.B.; Kalkwarf, K.L.; Patil, K.D.; Molvar, M.P.; Dyer, J.K. Long-term evaluation of periodontal therapy: I. Response to 4 therapeutic modalities. J. Periodontol. 1996, 67, 93-102. [CrossRef] [PubMed]

109. Mailoa, J.; Lin, G.H.; Khoshkam, V.; MacEachern, M.; Chan, H.L.; Wang, H.L. Long-term effect of four surgical periodontal therapies and one non-surgical therapy: A Systematic Review and Meta-Analysis. J. Periodontol. 2015, 86, 1150-1158. [CrossRef]

110. Singh, S.; Uppoor, A.; Nayak, D. A comparative evaluation of the efficacy of manual, magnetostrictive and piezoelectric ultrasonic instruments- An in vitro profilometric and SEM study. J. Appl. Oral Sci. 2012, 20, 21-26. [CrossRef] [PubMed]

111. Loos, B.G.; Needleman, I. Endpoints of active periodontal therapy. J. Clin. Periodontol. 2020, 47, 61-71. [CrossRef] [PubMed]

112. Manresa, C.; Sanz-Miralles, E.C.; Twigg, J.; Bravo, M. Supportive periodontal therapy (SPT) for maintaining the dentition in adults treated for periodontitis. Cochrane Database Syst. Rev. 2018, 1, CD009376. [CrossRef] [PubMed]

113. Wu, D.; Yang, H.J.; Zhang, Y.; Li, X.E.; Jia, Y.R.; Wang, C.M. Prediction of loss to follow-up in long-term supportive periodontal therapy in patients with chronic periodontitis. PLOS ONE 2018, 13, e0192221. [CrossRef] [PubMed]

114. Lindhe, J.; Westfelt, E.; Nyman, S.; Socransky, S.S.; Haffajee, A.D. Long-term effect of surgical/non-surgical treatment of periodontal disease. J. Clin. Periodontol. 1984, 11, 448-458. [CrossRef] [PubMed]

115. De Freitas, L.F.; Hamblin, M.R. Proposed mechanisms of photobiomodulation or low-level light therapy. IEEE J. Sel. Top Quantum Electron. 2016, 22, 7000417. [CrossRef] [PubMed]

116. Meisel, P.; Kocher, T. Photodynamic therapy for periodontal diseases: State of the art. J. Photochem. Photobiol. B 2005, 79, 159-170. [CrossRef] [PubMed]

117. Sbordone, L.; Ramaglia, L.; Gulletta, E.; Iacono, V. Recolonization of the subgingival microflora after scaling and root planing in human periodontitis. J. Periodontol. 1990, 61, 579-584. [CrossRef]

118. Carrera, E.T.; Dias, H.B.; Corti, S.C.T.; Marcantonio, R.A.C.; Bernardi, A.C.A.; Bagnato, V.S. The application of antimicrobial photodynamic therapy (aPDT) in dentistry: A critical review. Laser Phys. 2016, 26, 1-23. [CrossRef]

119. Fumes, A.C.; Romualdo, P.C.; Monteiro, R.M.; Watanabe, E.; Corona, S.A.M.; Borsatto, M.C. Influence of pre-irradiation time employed in antimicrobial photodynamic therapy with diode laser. Lasers Med. Sci. 2018, 33, 67-73. [CrossRef] [PubMed] 